Lh. 1588

SLAC-227

\title{
MASTER
}

\section{STRONG EFFECTS IN WEAK \\ NONLEPTONIC DECAYS}

Mark B. Wise

\author{
SLAC-Report-227 \\ April 1980
}

Prepared for the Department of Energy

under controct number DE-ACO3.76SF00515 
SLAC-227

(T/E)

STRONG EETECTS IN WEAK NOHLEPTONIC DECAYS"

Matk B. WIse

Stanford Linear Accelerator Cent.

Stanford University

stenford, colltornts 94305

Apr11 1980

Prepared for the Department of Energy

under contract number DE-Acn3-765F00515

Printed in the United States of Amergea. Availat 10 from the sat funnd Technlcul lnformat lon Servicu. W.s. Depariment of Comanerce. 5285 Por

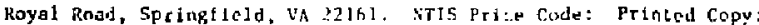

A07, Mexósthe: $A 01$.

\section{ABSTRACT}

Int thls report the weak nonleptonic decays of katung and hyperans are extined wlth the hope of gaining tristght into a recently proposed mechanisw for the $\Delta \mathrm{I}$ a $1 / 2$ rule. The effective Hamiltonian for $\Delta S=1$ weak nonleptonic decays and that for $\mathrm{k}^{\circ}-\overline{\mathrm{k}}^{\circ}$ atxing are calculated in the stx-quark model using the leading logarithmic approximation. These are used ro examine the CP violacton paraneters of the kaon system.

It is found that If Penguin-type diagrams make important contributions to $K+n \pi$ decay amplitudes then upcoming expertments way be able to distingulsh the s1x-quark todel for CP violation from the superweat model. The weak radiactive decays of hyperons are discussed whth an emphasis on what they can teach us about hypeton nonleptonic decays and the $\Delta 1=1 / 2$ rule.

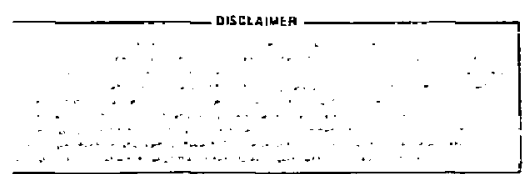




\section{ACKNOHLEDGE-GINTS}

I thank wy wife Jacquel ine whoge understanding ond encouragement magle thit report passtble. Mogt of che work discuased here was done In collaboration with $F$. J. Gilman and I am grateful to hiw for sharing some of $h x^{3}$ enorwous wealth of knowledge on hlgh energy physics with ine. I have alsa benefited frow collabarations uth $t$. F, abbotr,

P. Siklvie and E. Hitten. Finally 1 would like to thank my colleagues ac SLAC for many useful discusaions and for helpkng to make the study of high energy phyacs enjoyable.

\section{ABSTRACT}

ACEOHOHLEDGEIENTS

$\ldots \ldots \ldots \ldots \ldots \ldots$

TABLE of CONTENTS ..................... Iv

Chapser

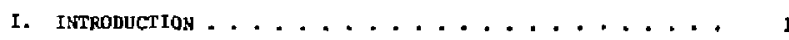

II. EFFECTIVE HAMILTONLAN FOR AS -1 WEAK MONLEPTOHIC DECAYS IN THE SIX-quARK MODEL ............ 18

1. Derivation of the Effectve Nooleptonle Heak

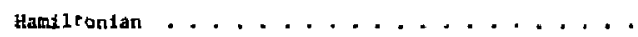

2. Numerical ResuIts for the Effective Nonleptualc

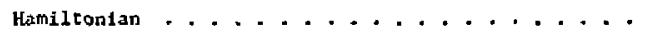

I11. EFFECTIVE haMILTONIAN FOR $x^{\circ}-\bar{x}^{\circ}$ MIXING IN THE

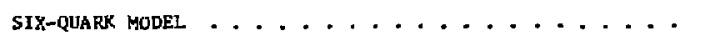

1. Derivition of the Effective Hamiltonian for $\mathrm{x}^{0}-\overline{\mathrm{x}}^{\mathrm{O}}$ Hexing .................... 46

2. Numetical Resules ............. 64

15. CP v lolation papameters of the $k^{\circ}-\overrightarrow{\mathrm{K}}^{\circ}$ systey ....... 69

1. Predfctions for $\varepsilon^{\prime} / \varepsilon \ldots \ldots \ldots \ldots$. . . . . .

V, WEMK RADLATILE HYPERON DECAYS .............. 84

1. The E/d Ratio In Nonleptonic Weak Hyperon Decays... 90

v1. CONCI.LDING reMarks ................... 94

APPEROIX A ............................. 96

APPENDIX в .............................. 105

APPENDIX $\mathrm{C} \ldots \ldots \ldots \ldots \ldots \ldots 116 \ldots \ldots \ldots$

REFERENCES ............................... 118 5 .

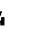

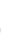

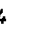

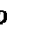

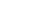
. s 118 
CHAPTER I

INTRODUCTION

The of the prominent features of the monleptonic weak decays of kaons and hyperons is the $\Delta I=1 / 2$ rule. The effective Hamiltonian for $\Delta S=1$ weak nonleptonte decays can be weitcen as the sum of 1 sospin $1 / 2$ and $3 / 2$ pteces. Experimentally it is ohserved that those decays which proceed through the lsospin $1 / 2$ part the effective Hamileonian ate enhanced by roughly a facter of 20 in amplitude over chose which proceed through the isosptn $3 / 2$ part of the e[fuctive Hamlltonlan. This is karrn as the $\Delta I=1 / 2$ rule. As an example consider kann decay into two ptons. The decay $k^{+} \rightarrow \pi^{+} \pi^{\circ}$ proceeds only through the $I=3 / 2$ pate of the effective Hamiltunian since the two-pion scate is charged and therefore musc have $\mathrm{I}=2$. The decay $\mathrm{k}_{\mathrm{S}}^{0}+\pi^{+}{ }^{-}$, on the other land, can proceed through both the $I=1 / 2$ and $I=3 / 2$ parts of the erfective Hamiltonian. Experimentially 1

$$
\frac{r\left(k_{\mathrm{s}}^{0}+n^{+}{ }^{-}\right)}{r\left(\mathrm{k}^{+}++^{+} \pi^{0}\right)}=450
$$

1n the standird 4-quark weinberg-5alam" model for weak and elcctromagnet ir interactions the quarks jre atsigned co right-handed singlets

$$
(w)_{R} ;(r)_{R}:(d)_{R}:(s)_{R} \quad(1.27)^{2}
$$

and 1oft-handed dantlets

$$
\left(\begin{array}{l}
4)_{1} \\
15
\end{array}\right)_{4}^{5}
$$

The fields di and ci are woak ingenstates and rulated in the mass elpenstates by a unitary rrangformaton. With an approprtate chotce for the quark fleld phases this transformation can be written in the following form

$$
\left(\begin{array}{l}
d^{\prime} \\
s^{\prime}
\end{array}\right)_{L}=\left(\begin{array}{cc}
\cos _{c} & \sin \theta \\
-\sin \theta_{c} & \cos \theta
\end{array}\right)\left(\begin{array}{l}
d \\
s
\end{array}\right)_{L}
$$

Q is called the Cabibbo angle. In the absence of strong interactions an effective Hamileonian for $\Delta S=3$ wesk nonleptonic decayg can be derived in this model by treating the H-boson mass as very large ard neqlecting the momentum trunsfer in the W-boson propagator. This ts 11lustrated in Fig. 1. The resulting ef fective Hamiltontan is the Eamilar local four-ferm fon $(V-N) \otimes(V-A)$ current-current Interactiun

$$
\begin{aligned}
\mathscr{W}_{\text {eff }}|\Delta S|=1 & =\frac{G_{F}}{\sqrt{2}} \sin \theta_{c} \cos \theta_{c}\left(\bar{s}_{c} \gamma^{\nu}\left(1-\gamma_{5}\right) u_{\alpha}\right)\left(\bar{u}_{B} \gamma_{H}\left(1-\gamma_{5}\right) d_{B}\right) \\
& -[u \rightarrow \varepsilon]+\text { h.c. }
\end{aligned}
$$

where $G_{F}$ is the Ferml constant and a and $B$ are color indices which are sumed ovet $\{1,2,3\}$ when repeated. It is conventent to decompore thls Hamflconian Into a sum of color symetric and color antisymutrie pieces In the following mannet

$$
\begin{aligned}
& x_{\text {efE }}^{|\Delta S|=1}=\frac{C_{F}}{2 \sqrt{2}} \sin { }_{c} \cos \theta_{c}\left[\left\{\left(\bar{s}_{a} \gamma^{U}\left(1-r_{5}\right) u_{\alpha}\right)\left(\bar{u}_{B} \gamma_{\mu}\left(1-r_{5}\right) d_{B}\right)\right.\right.
\end{aligned}
$$

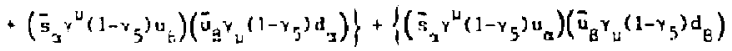

$$
\begin{aligned}
& \left.-\left(\bar{s}_{a} \gamma^{L}\left(1-\gamma_{5}\right) u_{g}\right)\left(\bar{u}_{g} \gamma_{u}\left(1-\gamma_{g}\right) d_{a}\right) !\right]-(u+c]+\text { h.c. } .
\end{aligned}
$$


The first set of brace brachets antiains a piece which is sume-

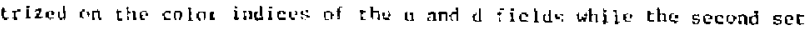
of bract brackets enntains a colot antisymetrized piece. the color ant isvmetric piece ts pure $I=1 / 2$ while the color symmetrte plece has an $I=1 / 2$ porcion and an $I=3 / 2$ portion. of course all the rems with charm and anti-charm quark fields are $\mathrm{I}$ - $1 / 2$ since the charnt quark has no isospin.

It was originally conjectured by wilson ${ }^{3}$ that strong interaction corrections would enhance the $I=1 / 2$ portion of the effective Haslltonian thus providing an explanatior for the Al $=1 / 2$ rule. With the advent of Quantum Chromodynamics (QCD) is it theory for the strong interactions such torrections became aalculable. Conslder. For example, the correctlon in Fig. 2a. If the momentum transfer in the k-boson rropaparor rould be neglected this iiagram would just give the arder $g^{2}$ (where ${ }^{\prime}$ is the serong cuupling) correction, shown in fig. $2 b$, to the matrix elements of the Iacal 4-quark operators in the effective Harileonian of Eq. (1.j), However, sine Fig. $2 b$ is ultravloletly divergent the ennverpience of the loop itutegral in Fite. $2 a$ is not good enough for such an approxination. If nue differentiates the amplitude, represenced by Fig. 23, with rispect to an external momentum the vitraviolet convergenct is tmproved enough so that the momentum cransfer can be ne'glected in the li-toson propagator. This means that Figs. 2a and 25. differ (tw) leading order in the large ti-boson mass) by a constent independent of the external monenen which is thus propnctional to che crece approximation for the matrix elenents if a local 4 -quark cperator. The constant of froportionaticy $A\left(x_{h} / 4,4\right)$ is also independent of the 


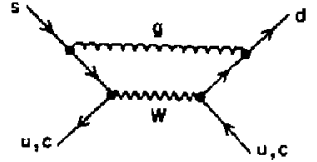

(a)

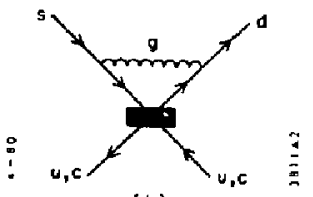

(b)

Fig. 2. (a) Higher order diagram contributing to weak $\Delta S=1$ nonleptonic decays.

(b) Higher order diagram contributing to the matrix -2ements of local four-quark operatorts In the effective Hamilzonian for $\Delta S=1$ nonleptontc weak decays. quark masses since the above argument can he repeated differentiating now with respert to quark masses. Other dlagrans cin be accommodited in a sirilar fashion the net result being that the effective Hamiltonian is a sum of color symmetric and color ancisymerte pitees, but with courficients. differing from theit free quark values. That is

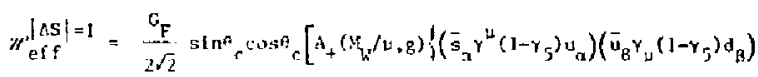

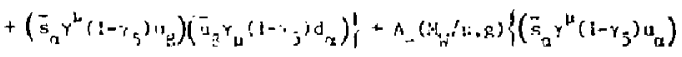

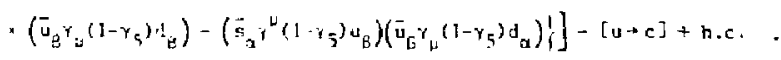

The functions $A_{1}\left(M_{b} / \mathrm{w},()\right.$ depend on the renarmalization scheme. of course, renormalization sehame dependence in the matrix elements of the operators must cancel this so that physical processes do not depend on choice of regularization scheme. W is the renormalization, point mass and dependence of the Wilson coefficients $A_{t}$ on it is likewise cancelled by the depenterce of the aitrix elentents on $\ldots$. The coefficients $A_{+}$and A_ thave been calculaced in the leaditg logarichmic aprroximation by Caillard and Let" and Altarelli and Mania." They found, for cypical values of the QCD parameters, that $i_{-}$was enhaneed (compared with $i$ trs

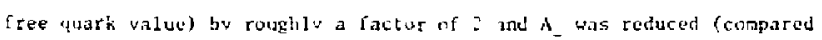
with its free quark value) by roughly the factor. $T$. While this result is in the correct direction to explain the $\mathrm{EI}=1 / 2$ rule it is much too sma1l in magnitude.

The b-boson mass is noc the only large mass scale in the problem. The charm quark mass is also "large" when compared with typical light 
hadronic masses. Thus one can inagine treating the charm quark at as

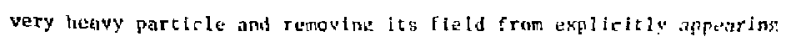
In the theory. Wher this is dont local a-quath operators with a shiral structure $(V-A) Q(V+A)$ will encer the Hatrileniat. Consider, for

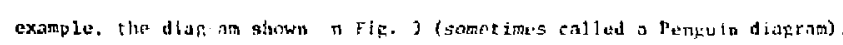
Calculation repeals that th the approximation of creating the h-bosson and charm quark as very heawy the loop integral gtyes a factor of $k^{2}$ which cancels the pole in the gluon propagatcr. As a resilt the anplitude corruspending to Fla. 3 can be reproduced by the true approximatiun to lie macrix elemerics of a lcal 4-quark operator involving only lighe v. $d$ and $s$ quark firlds. It is natural to wonder whether this local fove-fermion resule is an artifact of the lowesc arder coleulation o: will persist to hlgher urders. In Fig. 43 the foctor of $k^{2}$ from the uppor loop Integral cancels the gluon propagator (when the mysses of the light acrange and down quarks are zern). This, hovever, dies not lead to a lacal 4-quatk structure bat instead to a structura shown schemat teally in fip. 4h. Anoeher class of diagtams that wisht gecm to sirow that the local four-fermion result of fig. 3 is an artifact is shown in Fib. 5. Agnin diagrams of chis type do not admit an Interpresation ta cerms of a local fout-fermion structure. Moreover they ara no smaller than the lowest-urder diagrom even th the 3 imlt of large charm quark and W-bosan midsses. The dlagrams of Fig. 5 wuld, taken by chacmelves, ruin the lowest-order local four-fermion resule. Howger, When the contributions of Figs. 6a and 5 are adjed together $n$ cancellation of soft-gluon effeces occurs becween cbese diagrams so that thetr sum is included in the matrix elements of a local four-fermion operatcr

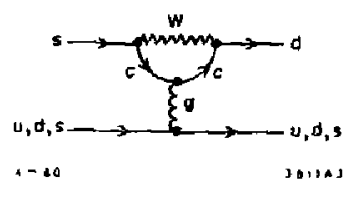

Ftg. 3. Lowest order Penguln-type diagram. 


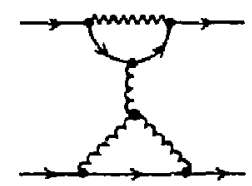

(a)

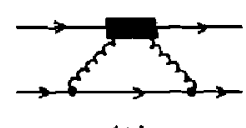

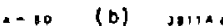

F1g. 4. (a) A two-loop Penguin-type dlagram,

(b) Symbolic representation of (a)

11lustrating the cancellation of a gluon propagator by the upper
loop Integration.
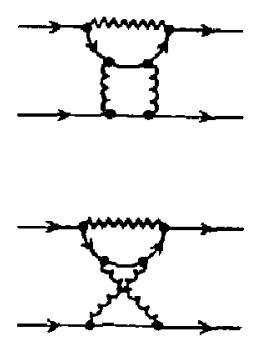

$-\infty$

311.45

F1g. 5. Two-loop Penguin-type diagrams which are not included in the matrix elcments of a local four-femlon operatar composed only of l lght $u, d$ and $s$ quark tields. 
constructed out of light-qquark tields. This cancollation is il result

of geuge invariance. It occurs berinse (for sorte gluons) the amplitudes corresponding to the diagrams is Fis. b which inntribute to tw proces: s $+d+$ gluons are reproduced dapat f fom gonstant plectus which will

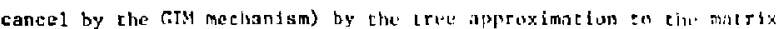
elements of the operator

$$
\begin{aligned}
& \text { r.Pengutn }=\frac{G_{F}}{\sqrt{2}} \sin { }_{c} \cos \theta c_{c} \frac{E^{2}}{12 \pi^{2}} \ln \left(\frac{m_{c}^{2}}{\omega^{2}}\right)
\end{aligned}
$$

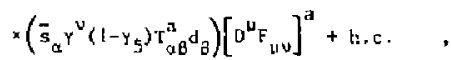

to leading order Ln the large mnsses. In $E q,(5.7)$ of is the strong coupling, $T^{2}$, a $[1,2, \ldots, 8\}$, are SU(3) color matrices nozmalized by $\operatorname{Tr}\left(T^{a} T^{b} y=\delta^{a b} / 2, F_{i v}^{A}\right.$ is the gluon fleld :itrength tensar and $D_{\nu}$ denotes a covartant derivative. Diazram ith more than three gluons attached to the quark loop are not important slnce thyy cannot produce a large logarithm In the c-quark mass. UEing the equations of motion for QCD

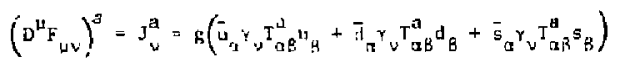

"Penguin becomes a local faur-fermion operator. From thits discussion it Is clear that canceluaions almilar to that between the diagrams in F1gs. 4 a and 5 wlll occur between other higher order dingrams so that the local four-fermion structure of the lowest order pengutn diagram in FlF. 3 will be preserved in the sense that the sum of all Penguin-cype diagrams, with arbitray ghuon ingertirns, equals a sum or wisnth coefficients times matrix elements of local 4-quark operators. Some of the operators inciuced by the Penguin-type diagrains will have a chiral

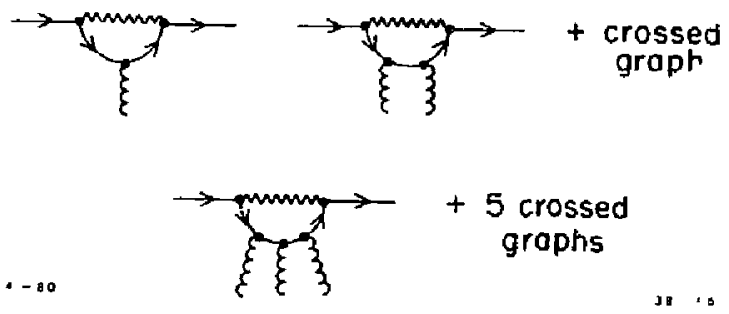

Fig. 6. One particle-irreducible diagrams contributing to the transition $s+d+$ gluons at the one-loop level. In the absence of strong Interactions there is a one-loop diagram that gives a $s+d$ transition hovever to leading orde $r$ in the d-boson aass it can be absorbed into mass rer.omalization. 
structure $(\mathrm{V}-\mathrm{A}) \Theta(\mathrm{V}+\mathrm{A})$ due to the vector coupling of the g.luons to quarks. Note also that all the Pengutn-type diagrans are pure $I=1 / 2$ since the gluon carries no 1sospin.

The efEect Ive Hamiltonian for weak $\Delta S=1$ nonleptenic decays, which results from successively treating the W-boson and charm nuatk as heavy flelds and removing them from explfcitly appearing in the theory, has been calculated in the leading logarithmic approximation 'y Shyfman, Vainshtein and Zakharov. ${ }^{9}$ They lound that tne speraters Indueed by the ?enguln-type dlagrams have snall wilson coeffichenes and ac firsc glance appear to make only an Insigaificant contribi:ilon to the weak nonleptonic decays of kuons and hyperons. However, the matrix elements of these operatora with a $(V-A) @(V+A)$ chiral structure may be greatly enbanced aver thoge of operators with the usual $(Y-A) \otimes(V-A)$ chiral structure. ${ }^{B}$ Such an enhancerent occurs, for exampic, when the matrix elements are evaluated by saturating the matrix element of a product of quark biligears With the vacum internedlate state, Since the $(V-A) Q(V+A)$ operators are pure $1=1 / 2$, combining the enhancement of the1r matrix elements with the enhancement of the Wilson coefricients of the $I=1 / 2$ conblnation of the famliar $(V-A) \otimes(V-A)$ operators may provide a qualitative explanation for the $\Delta I=1 / 2$ rule, Much of this report will be devoced to exrmining the consequences of this possible mechantsm for the $\Delta t=1 / 2$ rule and to teoting its validity.

The nonleptonic veak decays of the neurral kanns have another feature which 15 even mote strkking that the $\Delta I=1 / 2$ rule. They violate CP tnvartance. If $C P$ was conserved the phusteul neutral kaon elgenstazes $K_{S}^{0}$ and $k_{t}^{0}$ would be the CP eigenstates

$$
\left|k_{1}\right\rangle=\frac{\left|K^{o}\right\rangle+\left|\bar{K}^{o}\right\rangle}{\sqrt{2}}
$$

and

$$
\left|k_{2}\right\rangle=\frac{\left.\left.\mid k^{o_{3}}\right\}-\mid \bar{k}^{o_{3}}\right\}}{\sqrt{2}},
$$

with $\mathrm{CP}=+1$ and -1 respectively. Since a neutral two pion s-wave state has even CP a $\mathrm{K}_{2}$ cannot decay linto two plons :iten CP 1s congerved. However, expertmentally it is observed that ${ }^{3}$

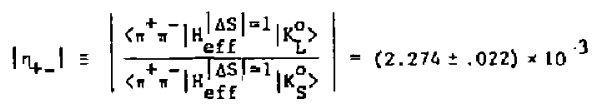

and

$$
\left|n_{00}\right| \equiv\left|\frac{\left\langle\pi^{\circ} \pi^{\circ}\left|H_{\text {efE }}\right| \Delta S|=1| k_{Z}^{O}\right\rangle}{\left\langle\pi^{0} \pi^{0}\left|H_{\text {eff }}\right| \Delta S|=1| k_{S}^{o}\right\rangle}\right|=(2.32 \pm .09) \times: 0^{-3} .
$$

The difference of $\eta_{+}$and $\eta_{00}$ fron zero is a measure of CP violation.

In the 4-quark WeInberg-Salan model with the manlmal HLggs sector (1.e., onc Higgs doublet) CP is conserved." However, as was pointed out by Kobryash1 and Maskava, 10 in the s:ix quark model with right handed \$inglets

$$
\left.(u)_{R} ;(c)_{R} ;(c)_{R} ;: d\right)_{R} ;(s)_{R} ;(b)_{R}
$$

and lef $c$ handed doublets

$$
\left(\begin{array}{l}
u \\
d
\end{array}\right)_{L}:\left(\begin{array}{c}
c \\
s
\end{array}\right)_{L} ;\left(\begin{array}{l}
c \\
b
\end{array}\right)_{L}
$$

there is enough ireeciom for $C P$ violation $c u$ cacur. This model has tecome popular because of the discovery of affth lepton," $T$, and 
a fifth quark, b. ${ }^{12}$ a sixth quark $i$ is expected, ${ }^{13}$ beting necessary for the (generalized) Gig mechunismit as weil as the cancelbicton of anomalies. ${ }^{\text {t5 }}$ The primed flelds 1 it Eq. (1.11b) are not mass eigenstates. but are related to the mass eigenstates $s_{L}$. d and $b_{L}$ by a unitary transformation, $v$, which for the scondard cholce of phases for the quark fields $1 s^{10}$

$$
\left(\begin{array}{l}
d^{\prime} \\
s^{\prime} \\
b^{\prime}
\end{array}\right)_{L}=\left(\begin{array}{ccc}
c_{1} & -s_{1} c_{3} & -s_{1} s_{3} \\
s_{1} c_{2} & c_{1} c_{2} c_{3}-s_{2} s_{3} e^{1 d} & c_{1} c_{2} s_{3}+s_{2} c_{3} e^{10} \\
s_{1} s_{2} & c_{1} s_{2} c_{3}+c_{2} s_{3} \mathrm{R}^{2 d} & c_{1} s_{2} s_{3}-c_{2} c_{3} e^{1 \delta}
\end{array}\right)\left(\begin{array}{l}
d \\
s \\
b
\end{array}\right)_{L},
$$

where $c_{1}=\cos \theta_{1}$ and $s_{1}=\sin 0_{1}$. The signs of the quark fleid. may be adjusted so that the shree cablbbo-type angles $\theta_{f}, f \in\{1,2,3\}$, al1 1he fo the first quadrant. ${ }^{16}$ Then the quadrant of 5 has phystcal significunce and cannot be thosen by convention.

In this mojed weak interactions lnvolving the charged hadronic current follow from the Lteraction tem la the Hamiliconian dengicy

$$
\mathscr{W}_{I}=\frac{8}{2 \sqrt{2}} J^{u^{+} H_{L}^{-}}+\text {h.c. }
$$

where $w_{\mu}^{-}$is the charged $w_{-b o s o n}$ tleld, $j_{\mu}^{+}$is the charged wgak curtent defined by

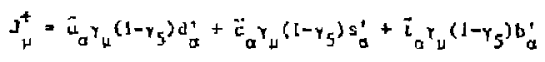

and 8 is the gauge coupling of the wink su(2) subgroup. Since the cP operator takes $a$ (nass eigenstate) quitk field into an anclquerk rield and $a W^{ \pm}$boson inca a $W^{+}$boson $C P$ wlll be rolated by this interaction Hantitontan if the phase of ts nonzero. Actually there are arbitrary phises in the deflatelon of the CP operator corresponding to th: arbitratiness of the choire of phases for the quark flelds. The correct statement ts that there will be CP violation if $1 \mathrm{f}$ is tmpossible by readjusting the phases of the quark fields to find a parametrization of the unitary matrix o (that relatos weak and masg elgengtater) whtch 1s purely real. In the 4-quark model uhere U is a $2 \times 2$ unitary matrix It is possible by readjustarg the phages of the quark fields to find the real parametrization given in cf. (1.3). However, in the six-quark model readjusting the phases of the quark flelds wi! just move che phase o eram one place in $U$ to another, but it can never be complecely removed from appearing $\mathrm{In}_{\mathrm{i}}$ (1.12).

The phenomenologicsl con asquences of the Kobayashi-Maskaw olxquark model for CP violation have been worked out by El11s, Garllard and Nanopoulos, 17 with strong interactions neglected, and were found to be consistent with experimenta. data on $\mathrm{K}$ decays. Part of this report is devoted to a study of the effects that strong interactiony have on the predictions which the stx-quark model makes for varkous tP vlolation parame, exs. In particular, che Penguin-type dlagrass, with beavy $c$ and $c$ quarks fr the loop have an imaginary CP violactig part and their CP violatiag contributions to $K+\pi \pi$ decay anplitudes arc dlscussed.

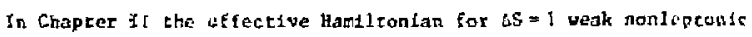
decays is computed in the o1x-quark model ustic the leading logaritinte approximation. In the follw ing chapter the effects of ged corretions un the $\mathrm{k}^{\circ}-\widetilde{\mathrm{k}}^{\mathrm{O}}$ mass matrlx are calculated. Chapter IV uses the results of thcse two calculations to make predictong for the co violation 
paramecers $n_{+}$and $n_{00}$ and in parcicular for the deviation of $n_{+-} / n_{00}$ from unley. It is shown that this devtation may be measurable if the Penguln-type dlagrams make Important contributions to the nonleptonfc decays of kaons. Chapter $v$ contains a brief discussion of ueak radiative hyperon decays. The weak radinttve decays of the negatively charged hyperons $a^{-}$and $\Xi^{-}$are particularly interesting since thev may proceed mostly through Penguin-type diagrams. Finally, Chapter vi contalns a brief sumury of results and some general canclustons are drawn.

\section{CHAPTER II}

EFFECTIVE RAMLLTONIAN FOR $: S$ - 1 WEAK NONLEPTONIC DECAYS IM THE SIX-QUARK MODEL ${ }^{19}$

In the standard $s i x$-quark model with charge $+2 / 3$ quarks $u, c$, and 5 and charge $-1 / 3$ quarks $d, s$, and $b$ the left-handed quarks are ass 1 gned to weak isospin dowblets and the right-handed quarks to weate Isospin singlets of the SU(2) $\odot \mathrm{V}(1)$ gauge kroup af weak and electrom sgnetic inceractions. The mixing berseen quarks tn doublets charncterlzed, say, by theit charge $+2 / 3$ members, is descrtbable by three cablbbo-like angles ${ }_{1}, 8_{2}$, and $s_{3}$, and by a single phase, 8 , which results in CP vlolotion. The nonluptonle weak interaction that cin resilt in a net changu in quark flavors is given to lowest order in weak tmteractions, ant zerocth order In strong Inecractions, by the producc a, a teak current of laft-handed quarks, it charked boson propagntur, ind anocher weak cursent of lefthajded ginaks. Siglecting the nomentum transfer dependence of the itbason propagator, one has the 11sthl local ( $(-A)$ D (V-A) structure of a currest-current weak nonle ponir banlltionian.

With she intruduction of strong interactions, in the fors of

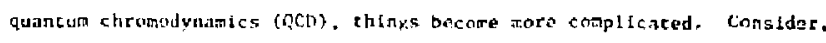
for example, that part of the andeptonle Hantlonidn responstble for decay of kanns and huperons which we weite in terms of the "likht" quarks $u, d$, and $\rightarrow$. As the strong interactions are turned on. not nnly is the

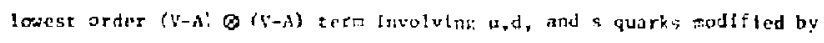
gluon exrhanqus betwer the quarks. Sut there are diagrass involvinz,

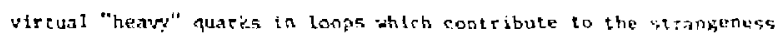
chanzing unleptosic Hanilmatan. These aleer the strenpt.. of the 
$(V-A) \otimes(V-A)$ tcrms and Incroduce rtew terms with different chiral structure, $a-\mathrm{B}_{-},(\mathrm{V}-\mathrm{A}) \otimes(\mathrm{V}+\mathrm{A})$.

It is the purpose of this chapter :o calculate the effective nonleptonlc Hantltonian for strarkencss changlng decays tn the six-quarh model. The b-bosor, t-quark, b-quark, and c-quark are successively considered as very heavy, and renotmalization group techniques $x=d$ to calculate (in the leading logarithmlc appreiztation) the resulfing effe-uve Hamliconian remalning at each stage.

the haste cechniques for carrying our such calculations have been latd out previously. 5,6, 8,19,20 They were even applied in the fourquark modal to get the effective liamilitontan for strangeness changing decays with the charm quark (and v-boson) taken as heavy. ${ }^{8}$ However, there is only one cablbbo angle in the four-quark model and in $\mathrm{CP}$ volacing phuse. It is the CP violnting pieces of the effective nonleptonic Haniltonian which are of special tnterest in this chapter.

In the next section the method by which the effective Hamiltonfan for nenleptonte strangenoss changing decays is to be calculated in the ax-quark model is deseribed. The approach is pedagoglcal and emphasizes the underly ing assugpttons and the condtions necessary for the valldity of the leadigg log approximation. In Section 2, numerical results are given. As expected, CP violating terms appear in the resulting effective Hamiltuatan, both in the old terms of $(V-A) \otimes(V-A)$ form and in the new "Penguln"-type terms. In the former they are quite smä11, but in the Latter are large. Many of the details concerning the matrices of anomalous dimenstonk and thekr elgenvectors and elgenvalues are relegated to an appendix.

\section{Dortuation of the Effectue Nonleptonte Heak Hamilitonlan}

Recall that in the standard model ${ }^{2}, 10$ where the gauge gru'up of weak and electromagnetic interactions is su(2) 0 g(1), the six quarks, $u, c$, and $t$ with charge $+2 / 3$ and $d, s$, and $b$, with charge $-1 / 3$, are assigned to left-handes doublecs and right-handed singlets:

$\left(\begin{array}{l}u \\ d^{\prime}\end{array}\right)_{\mathrm{L}} ;\left(\begin{array}{l}c \\ s^{\prime}\end{array}\right)_{\mathrm{L}} ;\left(\begin{array}{l}c \\ b^{\prime}\end{array}\right)_{L} ;(u)_{R} ;(d)_{R} ;(c)_{R} ;(s)_{R} ;(c)_{R}:(b)_{R}=$

is was mentioned in chapter I, the standard choies of quack flelds is guch that

$\left(\begin{array}{l}d^{\prime} \\ a^{\prime} \\ b^{\prime}\end{array}\right)_{L}=\left(\begin{array}{ccc}c_{1} & -s_{1} c_{3} & -s_{1} s_{3} \\ a_{1} c_{2} & c_{1} c_{2} c_{3}-s_{2} s_{3} e^{1 \delta} & c_{1} c_{2} s_{3}+s_{2} c_{3} e^{15} \\ s_{1} s_{2} & c_{1} s_{2} c_{3}+c_{2} s_{3} e^{L \delta} & c_{1} s_{2} s_{3}-c_{2} c_{3} e^{15}\end{array}\right)\left(\begin{array}{l}d \\ s \\ b\end{array}\right)_{L},(2.1)$

where $c_{1}=\cos \theta_{1}, s_{1}=3 \operatorname{tn} \theta_{1}, 1 \in\{1,2,3\}$. Equation (2,1) defines the three Cabibbo-like alxing angles $\theta_{1}$ and the GP violating phase, $\delta$.

Weak fateractions fnvolving the chacged hadrontc curtent follou from the Interaction term In the Hanltonlan densicy

$$
\mathscr{W}_{\mathrm{L}}(\mathrm{x})=\frac{\mathrm{g}}{2 \sqrt{2}} \mathrm{~J}^{+}(x) w_{\mu}^{-}(x)+\text { h.e. }
$$

where $w_{\nu}^{-}$in the charke $W$ boson field, $J_{\mu}^{+}$the charged weak current defined by

$$
\begin{aligned}
& J_{\mu}^{+}(0)=\bar{u}(0) \gamma_{\mu}\left(1-\gamma_{5}\right) d^{\prime}(0)+\bar{c}(0)_{\gamma_{u}}\left(1-\gamma_{5}\right) s^{\prime}(0) \cdot \bar{c}(0) \gamma_{\nu}\left(1-\gamma_{5}\right) b^{\prime}(0) \\
& \left.=\left(\overline{u d}^{\prime}\right)_{V-A}+\left(\bar{c} \bar{s}^{\prime}\right)^{V-A}+\left(\overline{\mathrm{E}} \mathrm{b}^{\prime}\right)\right)_{-A}
\end{aligned}
$$

and $g$ is the grage coupllag constant of the reak SU(2) subgroup. WIth 
no strong Inferactions the lowegt order weak current-current inturnction at zero momentum erangfer is doncribed by the effective Hamiltonian

densicy

$$
\mathscr{H}_{\text {ef }}(0)=\frac{g^{2}}{8 \mathrm{M}_{W}^{2}} \mathrm{~J}^{\mathrm{N}^{+}}\langle 0\rangle \mathrm{J}_{\nu}^{-}(0)+\text { h.c. }
$$

so that the Ferral coupling $C_{F} / \sqrt{2}=g^{2} /\left(g M_{\mathrm{H}}^{2}\right)$. In partlcular che strangt:nes 5 changing plece of Eq. (2.4) is

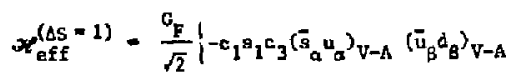

$$
\begin{aligned}
& +{ }_{1} c_{2}\left(c_{1} c_{2} c_{3}-s_{2} s_{3} e^{-1 \delta}\right)\left(\bar{a}_{a} c_{A}\right) v_{-A}\left(\bar{c}_{a} d_{B}\right)_{V-A} \\
& \left.+{ }_{1} s_{2}\left(c_{1} s_{2} c_{3}+c_{2} s_{3} e^{-i \delta}\right)\left(\bar{E}_{a} E_{a}\right) V-A\left(E_{B} d_{B}\right)_{V-A}\right\} .
\end{aligned}
$$

where the color indices $a$ and $B$ on the quarks (whych when repeated are sumed from 1 to 3) have been made expleft in preparation for the inclustor of the strong interactions. It is convenicnt to rewife Eq. (2.5) as

$$
\mathscr{X}_{\text {eff }}^{(\Delta S=1)}=-\frac{G_{E}}{2 \sqrt{2}}\left\{A_{c}\left(o_{c}^{(+)}+o_{c}^{(-)}\right)+A_{t}\left(o_{t}^{(+)}+o_{t}^{(-)}\right)\right\} .
$$

uhere

$$
\left.{ }_{q}^{( \pm)}=\left[\left(\bar{s}_{a} u_{a}\right)_{V-A}\left(\bar{u}_{B} d_{B}\right)_{V-A} \pm\left(\bar{s}_{a} d_{A}\right)_{V-A}\left(\bar{u}_{B} u_{B}\right)_{V-A}\right]-[u+q] \cdot(2 .)\right)
$$

$\operatorname{and}$

$$
\begin{aligned}
& A_{c}={ }_{1} c_{2}\left(c_{1} c_{2} c_{3}-s_{2} s_{3} e^{-1 \delta}\right) \\
& A_{t}=B_{1} \theta_{2}\left(c_{1} s_{2} c_{3}+c_{2} \theta_{3} e^{-1 \delta}\right)
\end{aligned}
$$

Nornal ordering of the four-fermion operators is understood. The spacetitoe coordinates of all opcracorg ate suppresged.

Now introfuce the strang Interactions in the form of quintur. chrowadynamics (QQCD), the gauge thuory based on the color SU(3) gauge roup involving vector gluon interacting with quarks." The strorg 1 teractions modify the lowest order weak effective HamiltonLan from the form in Eqs. (2.4) and (2.5). We now procecd to detive in leading lagarthmlc approxiascion the form of the effective weak Hamileonian in the presence of strong interactions vith heavy w-bosons and heavy $\tau, b$, and $c$ quirka.

First, the Wtoson is taken as ruch heavil. than any other pass scale in the problem and the s-racrix elements of the weak tatertetion between Iow momentum hadron states composed of 1 ight quarks and differing in scrangeness by one unlt are couraidered. Thas is jusf the calculatior performed In Refs. 5 and 6 . Using the operator prodtce expansion ${ }^{3}$ (noting that the operators $o_{c}^{(!)}$and $o_{l}^{(t)}$ are multiplicaclvely renozmalized and do not alx with other operators at thie one loop level) it follows that to leadfnp order in the heavy h-boson mass

$$
\begin{aligned}
& \left.\left.\left(-\frac{1}{2}\right) \int d^{4} x\left\langle\left|T\left(x-(x)+x_{I}(0)\right)\right|\right\rangle=-\left.\frac{G_{F}}{2 \sqrt{2}}\right|_{A_{c}^{(+)}} ^{\left(+H_{L}\right.}, g\right)<\left|0_{c}^{(+)}(0)\right|\right\rangle \\
& +A_{C}^{(-)}\left(\frac{M_{W}}{H}, g\right)<\left|0_{C}^{(-)}(0)\right|> \\
& +A_{t}^{(+)}\left(\frac{H_{H}}{\mu}, \mathrm{g}\right)\left\langle\left|0_{t}^{(+)}(0)\right|\right\rangle \\
& \left.+A_{t}^{(-)}\left(\frac{H}{H}, g\right)<\left|O_{t}^{(-)}(0)\right|\right) ! \text {. }
\end{aligned}
$$


where $\mu$ is the renormalfzatiol. polnt of the strong interactions. The mutrix elements of the right-hand stde are to be evaluated to all orders in the strong interactions (since perturbation theory is probably not valid) and to zeroech arder in the weak interactions.

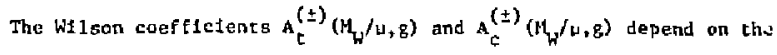
ehoice of renormalization scheme. Of course, matrix elements of the renormultzed operators $o_{t}^{(t)}$ and $o_{c}^{(\neq)}$also depend on the renomalization scheme in such a way that phystesl quantities are rundered scheme Independent. We use the mass Independent $\overline{M S}$ subtraction scheme $\overrightarrow{z t}$

where the renormalization group equatians 22 are

$$
\left(\mu \frac{\partial}{\partial L}+\beta(B) \frac{\partial}{\partial g}-\gamma^{(t]}(g)\right)_{A}^{( \pm)}\left(\frac{W_{W}}{\mu}, g\right)=0 .
$$

The $r^{(t)}$ characterize the anomalous dimension of the operacors $o_{q}^{(t)}$ with $q=c$ or $t$. The function $B(R)$ has the perturbation expansion: 23

$$
B(B)=-\left(33-2 \mathrm{~N}_{\mathrm{F}}\right) \frac{\mathrm{g}^{3}}{48 \pi^{2}}+C\left(\mathrm{~B}^{5}\right) \text {. }
$$

where $N_{E}$ (which equals 6 here) is the number of quark flavors. A Etandard one loop calculation 5.6 ahows that $\gamma^{(*)}(8)$ has the perturbation expansion:

$$
\begin{aligned}
& \gamma^{(+)}(8)=\frac{8^{2}}{4 \pi^{2}}+o\left(g^{4}\right) \\
& \gamma^{(-)}(8)=-\frac{g^{2}}{2 x^{2}}+o\left(g^{4}\right) .
\end{aligned}
$$

With the rundog coupling constant $\bar{g}(y, g)$ defined by

$$
\ln y=\int_{8}^{8} \frac{d x}{B(x)}
$$

and $\bar{F}(1,3)=\mathrm{B}, \mathrm{Eq},(2.10)$ has the solution

$$
A_{q}^{( \pm)}\left(\frac{M_{H}}{\nu}, 8\right)=\left[\exp ^{\overline{8}\left(M_{W} / L, g\right)} \int_{B}^{-\frac{Y^{( \pm)}(x)}{B(x)} d x}\right] A_{q}^{( \pm)}\left(1, \bar{B}\left(\frac{K_{W}}{W}, B\right)\right) .
$$

In a leading log enlcuiation the coefficients $A_{q}^{( \pm)}\left(1, \bar{B}\left(M_{1} / u, E\right)\right)$ can be replaced by thelt free fivld values ${ }_{q}$ glven $\ln E_{q} .(2.8)$ be-ause the ruming fine structure constant $a=\bar{g}^{2} / 4 \pi$ is small ac the mass scale of the $W$ and because the value of their [Lrse dependent varlable being unity implies no other large logarichas can be ketrerated by higinex otcler strong interactions. Using Eqs. (2.11) and (2.12)

$$
-\frac{y^{(t)}(x)}{B(x)}-\frac{2 a^{(t)}}{x}+\text { teras finite at } x=0,
$$

Hth

$$
\begin{aligned}
& a^{(+)}=\frac{b}{33-2 H_{f}} \\
& a^{(-)}=\frac{-12}{33-24 f_{f}}
\end{aligned}
$$

Choosing $u$ above the onsiet of scallng, Eq. (2.15) may be substituted back Into Eq. $(2,14)$ to obtain the result : ${ }^{4}$

$$
\begin{aligned}
& A_{q}^{(t)}\left(\frac{w_{H}}{H}, g\right)=\left[\frac{\bar{g}^{-2}\left(\frac{\mu_{u}}{\mu}, g\right)}{g^{-2}(1, g)}\right]^{(s)} A_{q} \\
& =\left[\frac{a\left(u_{w}^{2}\right)}{a\left(\mu^{2}\right)}\right]^{(s)} A_{q}
\end{aligned}
$$

At this gtage our effective vcak Hamiltonian density is 


$$
\begin{aligned}
& x_{\text {eff }}^{(\Delta 5-1)}--\frac{G_{F}}{2 \sqrt{2}}\left(\left[\frac{a\left(M_{L}^{2}\right)}{a\left(u^{2}\right)}\right]^{a^{(+)}}\left(A_{c} o_{c}^{(+)}+A_{c} o_{c}^{(+)}\right)\right. \\
& \left.+\left[\frac{\alpha\left(r_{L}^{2}\right)}{\alpha\left(\mu^{2}\right)}\right]^{a^{(-)}}\left(A_{c} o_{c}^{(-)}+A_{s} o_{E}^{(-)}\right)\right\} .
\end{aligned}
$$

The gatrix elements of the above effeclive weak llamilcontan donstty are to be evaluated to oll orders in the gtrong Interactions ant to zeroeth order in the wak Inceractions. Note thet eff daes nor expleficly Involve the $w$ boson fleld. He want co derive an afs tive Hamtitonian withouf explfcit dependence on the heavy $W$-boson, E-quark, b-quark and e-quark itelds. Equation (2.18) is the first step towards this gonl.

The nexc step in to consider the t-quark as very heavy and el iminate It from explteitly appearing in the effectue weak hambltonfan for strangeness changing processes. What happens to the operator $o_{c}^{( \pm)}$and $O_{t}^{(t)}$ is different, and the more complicated case of $0_{t}^{(\cdot)}$ is constuered flest.

We assume that mi 15 much greater than $n 11$ orher quatk masses, the pomenta of the external states, and the renomallzation point mass, 4 . The work of Appelquist and Carrazone imples that zo ocder $1 / \mathrm{m}_{\mathrm{t}}^{2}$ all the dependence of amplitudes on the heavy t-quark mass $L$ is be absorbed into renorhalization effects and hence inco a redefinition of the couphing constant, mass parameters, and seale of operators. This suggests the following factorization:

$$
\left\langle\left|0_{t}^{(t)}\right|\right\rangle=\sum_{1} B_{1}^{( \pm)}\left(\frac{\sigma_{1} t}{\omega} \cdot g\right)\left\langle\left|o_{1}\right|\right\rangle^{\prime}+\rho\left(\frac{t}{m_{t}^{2}}\right) .
$$

Where the primed ratrix elements are evaluated to all orders in an effective theory of strong Interactlong" with s-quatk flavors, coupling $g^{\prime}\left(m_{t} / u+g\right)$ and mass parameters $g_{11}^{\prime}, m_{d}^{\prime}, \ldots, m_{b}^{\prime}$. Thus,

$$
\left.\left\langle\left|\sigma_{i}\right|\right\rangle\right\rangle=\left\langle\left|a_{i}\right|\right\rangle^{\prime}\left(s^{\prime}, \nu, m_{u}^{\prime}, \ldots, m_{b}^{\prime}\right) .
$$

To carry out the expangton of Eq. $(2,19)$ in Leading, Iog approxtination six inearly independene operstors $O_{1}$ are suffielent. They are choosen as follows:

$$
\begin{aligned}
& 0_{1}=\left(\bar{g}_{a}{ }_{a}\right)_{v-A}\left(\bar{u}_{B} u_{B}\right)_{v-A} \\
& o_{2}-\left(\bar{g}_{d} d_{B}\right)_{v-A}\left(\bar{u}_{B} u_{a}\right)_{v-A} \\
& o_{3}-\left(\bar{a}_{d} d_{3}\right)_{V-A}\left[\left(\bar{u}_{B} a_{B}\right)_{V-A}+\ldots+\left(\vec{b}_{B} b_{B}\right)_{V-A}\right] \\
& o_{4}=\left(\bar{s}_{a} d_{B}\right)_{v-\lambda}\left[\left(\bar{u}_{B} u_{a}\right)_{v-\lambda}+\ldots+\left(\vec{b}_{B} b_{a}\right)_{v-\lambda}\right] \\
& o_{5}-\left(\vec{s}_{\alpha} d_{a}\right) y-A\left[\left(\vec{u}_{\beta} u_{\beta}\right) v+A+\ldots+\left(\vec{b}_{\beta} b_{\beta}\right) v+A\right] \\
& o_{6}=\left\langle\bar{B}_{a} d_{B}\right\} v-A\left[\left(\bar{u}_{B} v_{a}\right\}_{v+A}+\ldots+\left(\vec{b}_{B} b_{a}\right)_{v+A}\right]
\end{aligned}
$$

These operators are sufficlent stnce they close under renormal1zaclon at the nne loop leve?. The operators $o_{1}$ and $o_{2}$ already occur to zeroeth order In strong interactlonsi fr. follows fron Eq. (2. t) that

$$
\begin{aligned}
& B_{\}}^{( \pm)} \equiv B_{!}^{(t)}(1,0)= \pm 1 \\
& B_{2}^{( \pm)} \equiv B_{2}^{( \pm)}(1,0)= \pm 1
\end{aligned}
$$

The operators $O_{3}, O_{4}, O_{5}$, and $O_{6}$ aft nenerated by the strong interactions throvgh "Penguin"-type dtagrams, so that int free fitld theory 


$$
B_{3}^{(+)}=B_{4}^{( \pm)}=B_{9}^{(+)}=B_{4}^{(\cdot)}=0 .
$$

Hovever, the operaturs $o_{f}$ are not mulfiplicatively renormalized at the one loop level, 1.c., they $m 1 x$ among themselves. As show in the appendix, the renormalization group equation thelr cocffictent $B_{i}^{( \pm)}\left(\pi_{L} / u, g\right)$ satisfy is

$$
\begin{gathered}
\sum_{J}\left[\left(\mu \frac{\partial}{\partial \mu}+\beta(g) \frac{\partial}{\partial g}+Y_{t}(g) m_{t} \frac{\partial}{\partial m_{t}}+\gamma^{( \pm)}(g)\right) \delta_{i j}-Y_{i j}^{\prime T}\left(g^{\prime}\right)\right] \\
\cdot B_{j}^{( \pm)}\left(\frac{\mu_{t}}{H}, g\right)=0 .
\end{gathered}
$$

Here $y^{\cdot T}$ is the transpose of the anomalous dimension matrix of the operacors $O_{1}$ in the effectlve theory of scrong, interactions with 5 quarks and coupling $g^{\circ}$. It is the elgenvectors of $y^{\prime} T$ that correapend to operators which are multiplicatively renurmalized. The coefficient functions $\tilde{\mathrm{B}}_{1}^{( \pm)}\left(\mathrm{m}_{t} / w, \varepsilon\right)$ of these mulefplicatively renomal lzed operators are written as

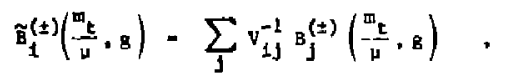

and the eigenvalues of $\gamma^{\prime}$ are denoted by $\gamma_{j}^{\prime}$. The matrix $\gamma^{\prime}$ is found In Appendix $A$ along with ftg elgenvalues and the natrix $v$. For the $\widetilde{B}_{i}^{(t)}\left(m_{t} / u, 8\right)$, the renormalization group equation corresponding to Eq. $(2.22)$ is

$$
\left(H \frac{\partial}{\partial \mu}+\theta(g) \frac{\partial}{\partial g}+r_{t}(g) a_{t} \frac{\partial}{\partial m_{t}}+\gamma^{(s)}(g)-r_{f}^{\prime}\left(g^{\prime}\right)\right) \tilde{\theta}_{i}^{(g)}\left(\frac{\Phi_{t}}{L}, g\right)=0 .
$$

The solution to this equacion may be found with the atd of the runnter coupling conscant $\bar{g}(y, g)$ def Ened by

$$
\ln y=\int_{8}^{\bar{g}(y, g)}\left[\frac{1-r_{t}(x)}{B(x)}\right] d x
$$

wtth $\overrightarrow{\mathrm{g}}(1, g)=\mathrm{g}$. Note that thls is not the usual definttion of the running coupling constant ( $\mathrm{E}_{\mathrm{q}},(2.13)$ ), but the Integrand in Eq. (2.25) for small $x$ has the same loading bchavior $g$ iven by $1 / A(x)$ as tiat integrand in Eq. (2.13). Settfng $v " m_{t} / \nu$, it is now aasily shown that the solution of Eq. $(2.24)$ is

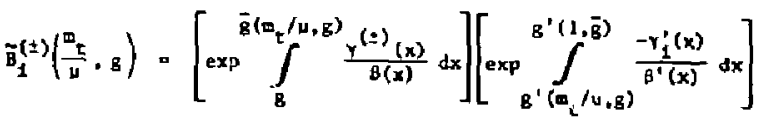

$$
\begin{aligned}
& \text { - } \tilde{\mathrm{E}}_{1}^{( \pm)}(1, \overline{8}) \text {. }
\end{aligned}
$$

$B^{\prime}$ is the beta furetion in the effective theory with 5 -quarks and coupling $g^{\prime}$. This beta function has the perturbation expanston

$$
B^{\prime}\left(g^{\prime}\right)=-\left(33-2 \mathrm{~N}_{f}\right) \frac{\mathrm{g}^{3}}{48 \mathrm{~s}^{2}}+O\left(\mathrm{~g}^{.5}\right)
$$

with $\mathrm{N}_{\mathrm{f}}=5$, and we wite

$$
-\frac{y_{i}^{\prime}(x)}{B^{\prime}(x)}-\frac{2 a_{1}^{\prime}}{x}+\text { finlte termg at } x=0 \text {. }
$$

Choosing $\mu$ as before, above the onset of scaling. Eqs. (2.15) and (2.28) may be used to get 


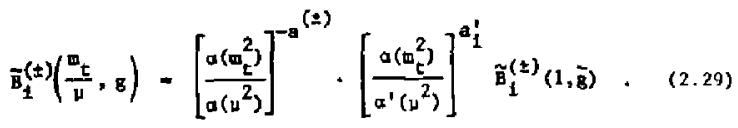

We have used $g^{\prime}(1, \bar{B}) \approx \bar{g}\left(m_{\tau} / \mu, g\right)$, which is valid in a leading log calculation ainec the running fine structure constant is small at the $\tau$-quark mass. Fins1ly, using the linear rele anghip berwcen the elgenvectors $\tilde{\mathbf{B}}_{i}$ and the $\mathrm{B}_{\mathrm{I}}$

$$
B_{k}^{( \pm)}\left(\frac{\Phi_{t}}{\mu}, 8\right)-\left[\frac{a\left(m_{e}^{2}\right)}{a\left(u^{2}\right)}\right]^{-a^{( \pm)}} \sum_{1, j} v_{k J}\left[\frac{a\left(a_{L}^{2}\right)}{a^{\prime}\left(\mu^{2}\right)}\right]^{a_{j}^{j}} v_{j 1}^{-1} B_{1}^{( \pm)}(1, \bar{g}) .
$$

Not1ce that the foctor $\left[a\left(\mathrm{~m}_{\mathrm{c}}^{2}\right) / \alpha\left(\mathrm{u}^{2}\right)\right]^{-\mathrm{a}^{(+)}}$out in front of the surmation In Eq. (2.30) combines with the earlier factor $\left[\alpha\left(M_{y}^{2}\right) / a\left(u^{2}\right)\right]^{a( \pm)}$ in Eq. $(2,16)$ to give $\left[a\left(\mathrm{~m}_{n}^{2}\right) / \mathrm{a}\left(\mathrm{m}_{\mathrm{s}}^{2}\right)\right]^{\mathrm{a}( \pm)}$. In leading log approximation the coefficlenta $B_{1}^{(\sharp)}(1, \bar{g})$ can be replaced by their free fleld values as given In Eq. (2.21), since no large logarithms can be generated from QCD loop integrals with the first argument of $B_{1}^{(t)}\left(m_{\tau} / \nu, g\right)$ set equal to unit; - because we assume the runing fine structure congtant is small at the t-quark mass.

The case of the operators $o_{c}^{( \pm)}$to auch simpler. The chara quark fleld which appears explictily in thege operators is of course aot difectly affected at thls stage of considering the t-quark as very heavy and the $o_{c}^{(t)}$ are Juat aultiplicatively renoraalized:

$$
\left\langle\left|0_{c}^{(t)}\right|\right\rangle-B^{(t)}\left(\frac{m_{t}}{\mu}, B\right)\left\langle\left|0_{c}^{(t)}\right|\right\rangle^{\prime}
$$

Note that the matrix elements on the right-hand side are agaln to be evaluated in the effective $f$ ive-quaik theory with coipling, $f^{\prime}\left(m_{t} / \mu, g\right)$. The coefficients $\mathrm{B}^{( \pm)}\left(\mathrm{m}_{\mathrm{c}} / \mathrm{w}, \mathrm{g}\right)$ satisfy

$$
\left(\mu \frac{\partial}{\partial u}+B(g) \frac{\partial}{\partial g}+\gamma_{t}(g) \nabla_{t} \frac{\partial}{\partial m_{t}}+\eta^{(t)}(g)-\gamma^{\prime( \pm)}\left(g^{\prime}\right)\right) B^{(t)}\left(\frac{m_{t}}{\mu} \cdot g\right)=0 .
$$

The anomalous dimenston $y^{( \pm)}\left(g^{\prime)}\right.$ is that of $o_{c}^{( \pm)}$and is a function of the coupling $g^{1}$ in the ef feetive five-quark theory, while $\gamma^{(:)}(\mathrm{g})$ depends on $R$, the coupling in the $s i x$-quark theory.

Solving Eq. (2.32) in the same manner as Eq. (2.24), gives

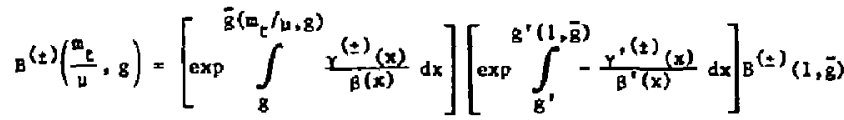

$$
\begin{aligned}
& -\left[\frac{a\left(m_{t}^{2}\right)}{a\left(p^{2}\right)}\right]^{-a^{( \pm)}}\left[\frac{a\left(m_{t}^{2}\right)}{a^{\prime}\left(u^{2}\right)}\right]^{a^{( \pm)}} B^{( \pm)}(1, \bar{B}) \text {. }
\end{aligned}
$$

In leading log appruxtinstinn $B^{(-)}\left(1, \bar{g}\left(m_{t} / \mu, g\right)\right)$ can be replaced by 1 ts free fleld value of +1 .

The effective wrak Hamlitonlan denstey is now free of explleft dopendence on the heavy t-quark field and has the form: 


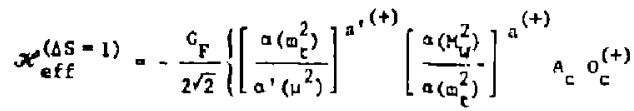

$$
\begin{aligned}
& +\left[\frac{a\left(\mathrm{~m}_{t}^{2}\right)}{a^{2}\left(\omega^{2}\right)}\right]^{a^{(-)}}\left[\frac{a\left(m_{\mathrm{t}}^{2}\right)}{a\left(\mathrm{~m}_{\mathrm{t}}^{2}\right)}\right]^{a^{(-)}} A_{c} o_{c}^{(-)} \\
& +\sum_{i}\left(\sum_{i, j} v_{k J}\left[\frac{a\left(a_{t}^{2}\right)}{a^{\prime}\left(\mu^{2}\right)}\right]^{a_{j}^{j}} v_{j i}^{-1} B_{i}^{(+)}\right)\left[\frac{a\left(m_{v}^{2}\right)}{\alpha\left(m_{t}^{2}\right)}\right]^{a(+)} A_{t} o_{k} \\
& \left.+\sum_{k}\left(\sum_{i, j} v_{k J}\left[\frac{a\left(m_{t}^{2}\right)}{a^{1}\left(\mu^{2}\right)^{2}}\right]^{a^{j}} v_{J 1}^{-1} B_{I}^{(-)}\right)\left[\frac{a\left(m_{k}^{2}\right)}{a\left(m_{t}^{2}\right)}\right]^{B^{(-)}} A_{t} O_{k}\right\}
\end{aligned}
$$

All operatore on the right-hand side are to have their patrix elements evaluated in the effective theory with five quarks, coupling $g^{\prime}\left(m_{\mathbf{r}} / \mathrm{u}, \mathrm{B}\right)$ and masses $m_{t}^{\prime}, m_{d}^{\prime}, \ldots, m_{b}^{\prime}$.

The pext step of constdering the b-quark as very heavy is simflar to wat, ras just accomplished for the t-quark, with the adjition of some indices. This time the marrix elements of the operators $0_{1}$ of $E_{q}$. (2.20) evaluated in the effective five-quark cheory are to be explessed in terms of matrix elemenes of

$$
\begin{aligned}
& P_{1}=\left(\overline{\bar{s}}_{a}{ }_{a}\right)_{v-A}\left(\bar{u}_{B} u_{B}\right)_{v-A} \\
& P_{2}-\left(\bar{B}_{a} d_{B}\right)_{V-A}\left(\bar{u}_{B}{ }_{a}\right)_{V-A} \\
& P_{3}=\left(\bar{s}_{\alpha}{ }_{\alpha}\right)_{V-A}\left[\left(\bar{u}_{B} u_{B}\right)_{v-A}+\ldots+\left(\bar{c}_{B} c_{B}\right)_{v-A}\right] \\
& p_{4}=\left(\bar{s}_{\alpha} d_{B}\right)_{V-A}\left[\left(\bar{u}_{B} u_{A}\right)_{V-A}+\ldots+\left(\bar{c}_{B} c_{Q}\right)_{V-A}\right]
\end{aligned}
$$

$$
\begin{aligned}
& P_{5}=\left(\bar{B}_{a} d_{a}\right)_{v-A}\left[\left(\bar{u}_{B} u_{B}\right)_{v+A}+\ldots+\left(\bar{c}_{B} c_{B}\right)_{v+A}\right] \\
& P_{b}=\left(\bar{B}_{a} d_{B}\right)_{v-A}\left[\left(\bar{u}_{B} u_{a}{ }{ }_{v+A}+\ldots+\left(\bar{c}_{B} c_{a}\right)_{v+A}\right]\right.
\end{aligned}
$$

evaluated in an effective theory with four quark flavors ( $u, d, s$, and $c$ ). The coupling and masses in the effective four-quark theory are denoted by $g^{\prime \prime}\left(m_{b}^{\prime} / u, g^{\prime}\right)$ and $\pi_{u}^{\prime \prime}, \ldots, \nabla_{c}^{\prime \prime}$, respectvely, io liadfng order in the b-quark mass

$$
\left\langle\left|o_{k}\right|\right\rangle^{\prime}-\sum_{a} c_{k}^{\prime}\left(\frac{w_{b}^{\prime}}{b}, g^{\prime}\right)\left\langle r_{n}\right\rangle^{\prime \prime},
$$

there the prime (double prime) denotes evaluation in the ef fective five (four) quark theory. The $C_{k}^{\mathbb{R}}\left(\mathrm{m}_{\mathrm{g}}^{\prime} / \mu^{\prime} g^{\prime}\right)$ can be shown to obey an equacion of the form

$$
\begin{aligned}
& \sum_{k, n}\left[\left(\mu \frac{\partial}{\partial \mu}+\beta^{\prime}\left(g^{\prime}\right) \frac{\partial}{\partial g^{\prime}}+\gamma_{b}^{\prime} g_{b}^{\prime} \frac{\partial}{\partial z_{b}^{\prime}}\right) \delta_{j k} \delta_{\operatorname{tm}}\right.
\end{aligned}
$$

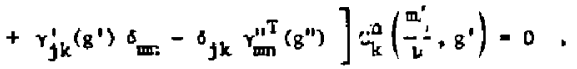

with $r^{\prime}$ and $r^{\prime \prime}$ being anomalous diengton atrices of the operators $0_{1}, \ldots, O_{6}$ and $P_{1}, \ldots, P_{6}$, respectively-

Defining the linear combinations of coefficient functions

$$
\tilde{C}_{k}^{n}\left(\frac{m_{b}^{\prime}}{\mu}, g^{*}\right)=\sum_{L} w_{n}^{-1} c_{k}^{2}\left(\frac{m_{b}^{\prime}}{\mu}, r^{\prime}\right)
$$

as corresponding to operators which are aultiplicatively renormalized, 1.e., do not wix ulth other operators, the renormalization group equationg diagoralize into the forw 


$$
\begin{gathered}
\left(v \frac{\partial}{\partial \mu}+\beta\left(g^{\prime}\right) \frac{\partial}{\partial g^{\prime}}+r_{b}^{\prime} \sigma_{b}^{\prime} \frac{\partial}{\partial m_{b}^{\prime}}+r_{k}^{\prime}\left(g^{\prime}\right)-r_{a}^{\prime \prime}\left(g^{\prime \prime}\right)\right) \\
\cdot \sum_{j} \tilde{c}_{j}^{\prime}\left(\frac{m_{b}^{\prime}}{\mu}, g^{\prime}\right) v_{j k}=0 .
\end{gathered}
$$

The martees $H$ and $\gamma^{\prime \prime}$ together with this e1genvalues of the latter are found in Appendix $\Lambda$.

With the ald of a seu runing coupling deffned by

$$
\ln y=\int_{g^{\prime}}^{\overline{8}^{\prime}\left(y, s^{\prime}\right)} \frac{1-y_{b}^{\prime}(x)}{s^{\prime}(x)} d x \text {, }
$$

these equations may be solved very anslogously to Eq. $(2,24)$, Leawing out some of the detalls, the solution in the leading logarichmic approxiration is

$$
\begin{aligned}
& c_{k}^{n}\left(\frac{m_{b}^{\prime}}{u}, g^{\prime}\right)=\sum_{1, i}\left(\sum_{j} v_{1 j}\left[\frac{a^{\prime}\left(a_{b}^{\prime 2}\right)}{a^{\prime}\left(g^{2}\right)}\right]^{-a_{j}^{\prime}} v_{j k}^{-1}\right) \\
& \text { - }\left(\sum_{m} w_{n=0}\left[\frac{a^{\prime}\left(m_{b}^{\prime 2}\right)}{\alpha^{\prime \prime}\left(w^{2}\right)}\right]^{a_{m}^{\prime \prime}} w_{m e}^{-1}\right) c_{1}^{L}\left(1, \bar{g}^{\prime}\left(\frac{m_{b}^{\prime}}{\mu}, g^{\prime}\right)\right) \text {. }
\end{aligned}
$$

For reasono scated before, In a leading lag calculation the coeffictents $c_{1}^{l}(1, \overline{8} ')$ can be replaced by their free field values:

$$
c_{i}^{\ell} \equiv c_{1}^{l}(1,0)=\delta_{f \ell} .
$$

The operators $0_{c}^{(t)}$ are multiplicatively renormalized and the expansion of thelr matrix elements gives results like those in $\mathrm{Eq}$. (2.31) with approptlate changes.
The effectue Hambltontan now takcs the followine form at the four-quark level:

$$
\begin{aligned}
& x_{e f f}^{(\Delta S-1)}=-\frac{G_{F}}{2 \sqrt{2}}\left\{\left[\frac{a^{\prime}\left(m_{b}^{2}\right)}{a^{\prime \prime}\left(u^{2}\right)}\right]^{a^{\prime \prime}(+)}\left[\frac{a\left(m_{t}^{2}\right)}{a^{\prime}\left(m_{b}^{\prime 2}\right)}\right]^{a^{\prime}(+)}\left[\frac{\alpha\left(m_{v}^{2}\right)}{a\left(m_{t}^{2}\right)}\right]^{a^{(+)}} A_{c} o_{c}^{(+)}\right.
\end{aligned}
$$

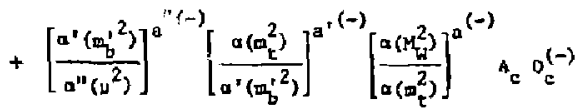

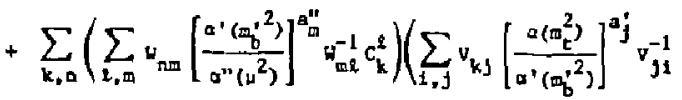

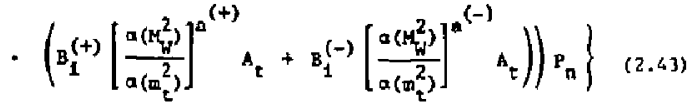

The final step of considering the chara quark as very heavy is more questionable from the phenomenological viewpoint. It also involves a teclenical point whtsh 16 easy to miss, When the natrdx elenents of che operators $P_{1}, \ldots P_{6}$ evaluated in the efferclup four-quark theory are expandec In terms of matrix elements of operatnra evaluaced in an affectlve three-quark theory, it is natural to define

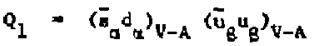

$$
\begin{aligned}
& Q_{2}=\left(\bar{a}_{a} d_{B}\right)_{V-A}\left(\bar{u}_{B} u_{a}\right)_{V-A} \\
& a_{3}=\left(\bar{s}_{a}^{d}\right)_{V-A}\left[\left(\bar{u}_{B} u_{B}\right)_{V-A}+\left(\bar{d}_{B} d_{B}\right)_{V-A}+\left(\bar{B}_{B} s_{B}\right)_{V-A}\right] \\
& Q_{4}=\left(\bar{s}_{a} d_{B}\right)_{v-A}\left[\left(\bar{u}_{B} u_{a}\right)_{v-A}+\left(\bar{d}_{B} d_{a}\right)_{v-A}+\left(\bar{s}_{z} s_{a}\right)_{v-\lambda}\right]
\end{aligned}
$$




$$
\begin{aligned}
& Q_{5}=\left(\bar{s}_{Q} d_{Q}\right)_{V-A}\left[\left(\bar{u}_{B} u_{B}\right)_{V+A}+\left(\bar{d}_{\beta} d_{B}\right)_{V+A}+\left(\bar{s}_{\beta} s_{B}\right)_{V+A}\right] \\
& q_{G}=\left(\bar{s}_{a} d_{B}\right)_{V-A}\left[\left(\bar{u}_{B} u_{\alpha}\right)_{V+A}+\left(\bar{d}_{B} d_{\alpha}\right)_{V+A}+\left(\bar{s}_{B} s_{Q}\right)_{V+A}\right]
\end{aligned}
$$

These operators close under renormalization at the une-1nop leve 1 , bue they are If nearly dependent:

$$
Q_{4}=-Q_{1}+Q_{2}+Q_{3}
$$

Hence only the 5 operators $Q_{1}, Q_{2}, Q_{3}, Q_{5}$ ard $Q_{6}$ are neceasary.

Expressing matrix elements of the operacors evaluated in the effective four-quark theory in terms of matrix elements of operators evaluated in the effective three-quark theory,

$$
\left\langle\left\langle P_{n} \mid\right\rangle^{\prime \prime}=\sum_{r=1,2,3,5,6} D_{n}^{r}\left(\frac{a_{c}^{\prime \prime}}{\mu}, B^{\prime \prime}\right)\left\langle\left|Q_{r}\right|\right\rangle^{\prime \prime}+r\left(\frac{1}{a_{c}^{2}}\right) \cdot(2.46)\right.
$$

जch $g^{\prime \prime \prime}$ and $m_{u}^{\prime \prime \prime}, m_{d}^{\prime \prime \prime}, m_{s}^{\prime \prime \prime}$ repregenting the coupling constant and quark masses in the effect1ve three quark theory. The linear combinations

$$
\tilde{D}_{L}^{I}\left(\frac{D_{c}^{\prime \prime}}{\mu}, g^{\prime \prime}\right)=\sum_{B} x_{x \in}^{-1} p_{n}^{g}\left(\frac{d_{c}^{\prime \prime}}{\mu}, g^{\prime \prime}\right)
$$

are the coefficients of multiplicatively renomaltzed operators. The d1agonalized remorwalization group equations are

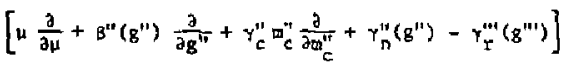

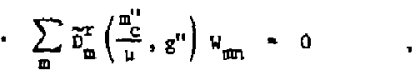

and have the solution in leading logarithaic approximation after re- expresging the D's in teras of $\mathrm{D}^{\prime} \mathrm{s}$,

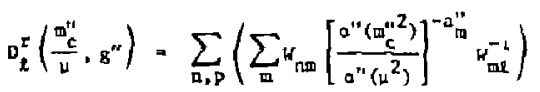

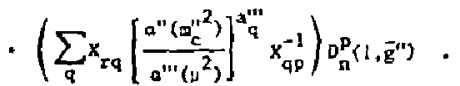

Io leading log approxiastion the $D_{a}^{P}\left(1, \vec{g}^{*}\right)$ can be replaced by thetr free fleld values, $D_{n}^{p}$. These are $d_{n p}$ except when $n-4$, in which case $D_{4}^{1}=-1$, $D_{4}^{2}=1, D_{4}^{3}-1$, and $\nabla_{4}^{5}=D_{4}^{6}=0$.

Because the charm quark is befing constdered as heavy, the operators $o_{c}^{(t)}$ are no longer just multiplicacively renormalized at the one loop level - It 15 also necessary to expand

$$
\left\langle\left|a_{c}^{( \pm)}\right|\right\rangle^{\prime \prime}-\sum_{r} D_{r}^{(t)}\left(\frac{m_{c}^{\prime \prime}}{\mu}, g^{\prime \prime}\right)\left\langle\mid Q_{r}\right\rangle^{m} .
$$

The renotwalization group equations obeydd by the $D_{r}^{( \pm)}\left(\Phi_{c}^{\prime \prime / \nu, g ")}\right.$ are

$$
\begin{aligned}
& \sum_{\mathbf{r}}\left[\left(u \frac{\partial}{\partial u}+\beta^{\prime \prime}\left(g^{\prime \prime}\right) \frac{\partial}{\partial g^{\prime \prime}}+\gamma_{c}^{\prime \prime}\left(g^{\prime \prime}\right){\varpi_{c}^{\prime \prime}}_{c} \frac{\partial}{\partial m_{c}^{\prime \prime}}+\gamma^{\prime \prime}()^{\prime \prime}\left(g^{\prime \prime}\right)\right) \delta_{p r}-\gamma_{p r}^{\prime \prime I}\left(g^{\prime \prime \prime}\right)\right] \\
& \text { - } \nabla_{I}^{( \pm)}\left(\frac{m_{C}^{n}}{\mu}, g^{\prime \prime}\right)=0 \text {. }
\end{aligned}
$$

The coefficients carresponding to wultipl featively renormalized operatarg are Just as $\ln$ E⿰氵..$(2.47)$, and the solution to Eq. (2.51) with the usual approximartions is

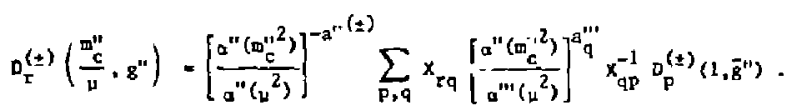


The frec fletd values, $\mathrm{v}_{\mathrm{p}}^{( \pm)} \equiv \mathrm{D}_{\mathrm{p}}^{( \pm)}(1,0)$, tre $\mathrm{D}_{1}^{( \pm)}=1, \mathrm{D}_{2}^{( \pm)}=+1$, and all others zero.

Finully, collecting all the results ti. : n-viously advertised effective Hadlitonian in the "light" three-quark sector can be uritten. It is the following sum of Wilson coefficients times locol four-fermion operators which do not explicitly Involve the heavy H-boson, top, bottom, and chatem quark ficlds:

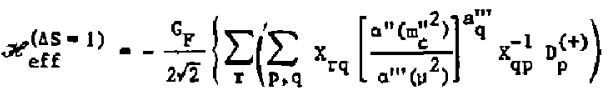

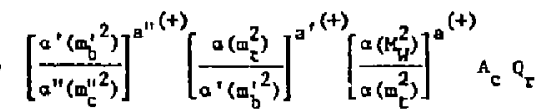

$$
\begin{aligned}
& +\sum_{x}\left(\sum_{p, q} x_{r q}\left[\frac{\alpha^{\prime \prime}\left(m_{c}^{\prime 2}\right)}{\alpha^{\prime \prime \prime}\left(\mu^{2}\right)}\right]^{a_{q}^{\prime \prime \prime}} x_{q p}^{-1} p_{p}^{(+)}\right) \\
& \text {- }\left[\frac{a^{\prime}\left(m_{b}^{\prime 2}\right)}{a^{\prime \prime}\left(m_{c}^{\prime 2}\right)}\right]^{a^{\prime \prime}(-)}\left[\frac{a\left(m_{t}^{2}\right)}{a^{\prime}\left(m_{b}^{\prime 2}\right)}\right]^{a^{(-)}}\left[\frac{a\left(m_{t}^{2}\right)}{a\left(m_{t}^{2}\right)}\right]^{a^{(-)}} A_{c} Q_{r} \\
& +\sum_{k, \pi, \tau}\left(\sum_{p, q} x_{r q}\left[\frac{a^{\prime \prime}\left(m_{c}^{\prime \prime 2}\right)}{a^{\prime \prime \prime}\left(\mu^{2}\right)}\right]^{a_{q}^{\prime \prime \prime}} x_{q P}^{-1} D_{n}^{p}\right)
\end{aligned}
$$

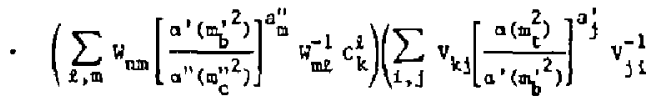

$$
\begin{aligned}
& \text { - } \left.\left.\left(B_{1}^{(+)}\left[\frac{a\left(m_{W}^{2}\right)}{a\left(a_{E}^{2}\right)}\right]^{a^{(+)}} A_{t}+B_{1}^{(-)}\left[\frac{\alpha\left(M_{W}^{2}\right)}{a\left(\pi_{t}^{2}\right)}\right]^{a^{(-)}} A_{t}\right)\right) Q_{r}\right\} \cdot(2.53
\end{aligned}
$$

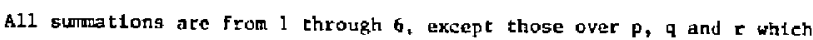
run through $1,2,3,5$ and 6

\section{Numerical Results for the Pffective Nonleptonic Hamiltonian}

It is nov possible to perform the arithmetic operncions mado explicit in Eq. (2.53) and to exambe the resulting Wilson coeffictents of the operators $\nabla_{1}, Q_{2}, Q_{3}, Q_{5}$ and $Q_{6}$ in the effective Hamilitantan for nonleptonic, strangeness changing Interactions. Since the matrlcas, $V, w$ and $X$, as glven tn the appendix, are composed of irrational numbers and since various fractional powers of $a\left(y^{2}\right)$ with $M^{2}=M_{W}^{2}, m_{t}^{2}$, cce, are rampant, quancitatively rather little is transparent about these coefficients in general. He then are forced to proceed by choosing a parametrization for $a\left(H^{2}\right)$ and values for the $w$ and quark masses, substicucing in Eq. (2.53), and rearing of $f$ the coefelctents of the $Q_{L}$ for chat particular set of choices.

Moreover, the out fook is basically qualitative. The QCo effects have been calculated in the leading log approxination. While wa have some confidence that at the $f(r s t$ step $i t, 1 s$ a large enough nags for chis to be a credible procedure, hy the last scep of considering $m_{4}$ a heavy mass this approximaclon has been used beyond the region where it can be reasonably justified.

On the positlve slate, what ts carried out here is upll defined and systematic. The degree of accura.y is obviously no worse than any of iite earlier calculatlons ${ }^{2}$ which involve only the "heavy" charm quark (and $w$ boson) in leading log approximation. Not only is the accuracy of the calculation uxpected to be betcer for the b and $t$-quarks, but 
their effect was not taken fnto account previously, With regard bo CP violation they play a dominant role.

To invegtigace the cffuctive nonleptonic Hamitconian numerically we flrst of all need to decide on the ruming QCD fine structure constant $a\left(Q^{2}\right)$, the values of thu heavy quark masses, and $\mu^{2}$ or alternately $a\left(\mu^{2}\right)$ In leading log approximation

$$
a\left(Q^{2}\right)-\frac{12 \pi}{33-2 N_{f}} \frac{1}{\ln \left(Q^{2} / \Lambda^{2}\right)},
$$

where we take $\Lambda^{2}=0.1 \mathrm{GeV}^{2}$ and $\Lambda^{2}=0.01 \mathrm{GeV}^{2}$, values consistent with recent daca when QCD is used to parametrize the breakdoun of scale invartance in deep inclastic neutino scattering. 27 When the leading log approximgtion 1s valid, the calculation is insensitive to the prectse value of $A$ and the difforence between $A^{+} s$ tn the vartous effectlve field theorles can be neglected. The number of quark flavors $1 s \mathrm{~N}_{\mathrm{F}}=6$ for the fine $5 t r$ ture const, nt we tave called $\alpha\left(Q^{2}\right)$, while $a^{\prime}\left(Q^{2}\right), a^{\prime \prime}\left(Q^{2}\right)$, and $\alpha^{\prime \prime \prime}\left(Q^{2}\right)$ have $N_{f} * 5 .^{\prime}$ and 3 respectively, as they pertaln to effective theorles with thase corresponding nutbers of quark flavors.

$m_{c}$ is taken to be $1.5 \mathrm{cry}$ and $m_{b}$ to be $4.5 \mathrm{GeV}$ on the basis of $\psi$ and $T$ spectroscopy. ${ }^{2 B}$ The $t$-quark mass is unknown at chis $r$ ine, and values of $35 \mathrm{GeV}$ and 30 Gev are used to get an idea of the sensitivity of the results to this quantity. For $w_{w}$ the value $85 \mathrm{GeV}$ is taken. In evaluating Eq. (2.53), $m_{b}^{\prime}$ Jnd $m_{b}, m_{c}^{\prime \prime}$ and $m_{c}$, are nor dist Inguished between, again consictent with the leatlng log approximation philosophy. Einally a value is requtred for $a\left(p^{2}\right)$ (or more exacrly $a$ "' $\left(u^{2}\right)$ ). We want to choose $\mu$ to be a typicol "light" hadron thass scale or inverse size, where $a\left(u^{2}\right)$ fs of order unity. He let o $\left(u^{2}\right)=0.75,1.0$ and 1.25 to check the variation of the reselting offective nonleptonic Hamilontan to this choice. In fact, the valueg of 5 -matrix elements of the weak inceraction carnot denend on the thatce of the renormalization polnt $u$, or equivalenty $\left.x f^{2}\right)$. The matrix elements of the four-ferminn opetators. $Q_{1}$, a1so have an implicic a denendence which exactly compunsates that of their coefficients (at lease wilun the coefficients are computed exactly). We are loft to make a choice of is, hopefully close to the cyptcal light hadron mass scale of the problem, so thet "hatd" gluon effects are ontalned as mueh as possible in the bilson coefficients ond not the matrix elements of $q_{1}$, bur high enough that their calcularion in leading $\log$ approxituation makes some seusu, 29

In terns of the operators. $Q_{1}, Q_{2}, Q_{3}, Q_{5}$ and $Q_{6}$ defined prevtously In Eq. (2.44). the nonleptonic Hamiltconlan lnvolving u,d and $s$ quark flelds has the form:

$$
\begin{aligned}
\boldsymbol{*}_{\mathrm{eff}}^{(\Delta S-1)} & =-\frac{G_{F}}{\sqrt{2}} s_{1} c_{1} c_{3} \mid\left(-0.87+0.036_{\tau}\right) Q_{1} \\
& +(1.51-0.036 \tau) Q_{2} \\
& +\left(-0.021-0.012_{\tau}\right) Q_{3} \\
& +(0.011+0.007 \tau) Q_{5} \\
& \left.+(-0.047-0.072 \tau) Q_{6}\right\}
\end{aligned}
$$

when $m_{t}=15$ GeV and $a\left(u^{2}\right)=1$ and where

$$
\tau-s_{2}^{2}+s_{2} c_{2} s_{3} e^{-1 t / c_{1} c_{3}} .
$$

ai ng with the other masses sperifled prevlously, Valties of the coeffictencs for all six cases correspondtng to $a\left(u^{2}\right)=0.75,1,0$ and 1.25 
and $m_{t}=15 \mathrm{GeV}$ and $30 \mathrm{GeV}$ are found in Table $I$ for $\Lambda^{2}=.1 \mathrm{GeV}$ and Table II for $\Lambda^{2}=.01 \mathrm{Gav}^{2}$.

Referring back to Eq. (2.5), it is apparent that before accountlag Eor the clfects of QCD, the coeffictents of the usual four-termiton operator $Q_{1}$, as well $n$ s the "Pengutn" Induced operators $Q_{3}, Q_{5}$ and $Q_{6}$ were all zero. In the sector Involving $u, d$ and $s$ qunrks the gtrangeness changing weak Hamflconian then just involves $Q_{2}$ with unif coefficient. Thus the prestence of atrong interaction QCD correctlons has brought in the opreatorg $Q_{1}, Q_{3}, Q_{5}$ and $Q_{6}$, changed the coefftctent of $Q_{2}$, and given all cocfficlents an limglnary (CP vlolating) pare chrough the quantity $\tau$, which enters through "Penguin"-type dtagrams Involving a henvy quark loop.

The portion of the nonjeptonfe Hamiltonian involveng only the operators $Q_{1}$ and $Q_{2}$ is the traditionally calculated $(V-A) \otimes(V-A)$ fourfermian plece with neglect of all "Penguin" effects. The sum of coefficients of $Q_{1}$ and $Q_{2}$ is proportional to the couffictont of $n$ operator transforming purely as $I=3 / 2$, whych cannot $\mathrm{mix}$ under strong interaction renormalization with "Pengutn" contributions which are pure $I=1 / 2$. As a consequence, one simple check of the calculation is to note that the quantity $\tau$, Rristng from "Penguin" contributions, alvays has the same magnitude and opposite sign in its contribution to the co s. Lcients of $Q_{1}$ and $Q_{2}$.

The combination of operacors $Q_{2}-Q_{1}$ transforns purely as $1=1 / 2$, whl le the combinaltar $Q_{2}+Q_{2}$ has an $I=3 / 2$ piece. The ratio of coefficients of $Q_{2}-\eta_{1}$ and $q_{2}+\eta_{1}$ is a measure of $d \tau=1 / 2$ or octer enhancenent by QCD, as fir.t calculated in Refs, 5 and 6 . The inclusion
TABLEE I

Coeffictents of the operators $Q_{1}, Q_{2}, Q_{3}, Q_{5}$ and $Q_{6}$ defined in $E q$. (ïj) in the effectiva Hadiltodian, $x_{\text {eff }}=\left(-G_{F} B_{1} c_{1} c_{3} / \sqrt{2}\right)\left(\sum_{1} c_{1} Q_{1}\right)$, for strangeness chsog Ing, goafeptonle weak decays. $\tau \equiv 8_{2}^{2}+\sigma_{2} c_{2} s_{3} e^{-1 \delta} / c_{1} c_{3}$. $A^{2}=.1 \mathrm{cev}^{2}$.

\begin{tabular}{|c|c|c|c|c|c|}
\hline Parametera & $c_{1}$ & $c_{2}$ & $c_{3}$ & $c_{5}$ & $c_{6}$ \\
\hline $\begin{aligned} a\left(\omega^{2}\right) & =0.75 \\
m_{c} & =15 \text { GeV }\end{aligned}$ & $\begin{array}{l}-0.72 \\
+0.035 r\end{array}$ & $\begin{array}{l}+1.40 \\
-0.035 x\end{array}$ & $\begin{array}{l}-0.013 \\
-0.015 r\end{array}$ & $\begin{array}{l}+0.001 \\
+0.008 \tau\end{array}$ & $\begin{array}{l}-0.025 \\
-0.059\end{array}$ \\
\hline $\begin{aligned} a\left(w^{2}\right) & =1.00 \\
m_{t} & =15 \mathrm{GeV}\end{aligned}$ & $\begin{array}{l}-0.87 \\
+0.036 \mathrm{r}\end{array}$ & $\begin{array}{l}+1.51 \\
-0.0367\end{array}$ & $\begin{array}{l}-0.021 \\
-0.0121\end{array}$ & $\begin{array}{l}+0.011 \\
+0.007 \mathrm{~T}\end{array}$ & $\begin{array}{l}-0.047 \\
-0.0727\end{array}$ \\
\hline $\begin{aligned} a\left(u^{2}\right) & =1.25 \\
a_{t} & =15 \mathrm{Cev}\end{aligned}$ & $\begin{array}{l}-1.00 \\
+0.036 x\end{array}$ & $\begin{array}{l}+1.61 \\
-0.0367\end{array}$ & $\begin{array}{l}-0.028 \\
-0.010\end{array}$ & $\begin{array}{l}+0.015 \\
+0.0067\end{array}$ & $\begin{array}{l}-0.069 \\
-0.085\end{array}$ \\
\hline $\begin{aligned} a\left(u^{2}\right) & =0.75 \\
w_{i} & =30 \mathrm{GeV}\end{aligned}$ & $\begin{array}{l}-0.71 \\
+0.042 \mathrm{~T}\end{array}$ & $\begin{array}{l}+1.39 \\
-0.042 t\end{array}$ & $\begin{array}{l}-0.013 \\
-0.017 \pi\end{array}$ & $\begin{array}{l}+0.007 \\
+0.009 \mathrm{r}\end{array}$ & $\begin{array}{l}-0.025 \\
-0.076\end{array}$ \\
\hline $\begin{aligned} a\left(u^{2}\right) & =1.00 \\
a_{t} & =30 \mathrm{GeV}\end{aligned}$ & $\begin{array}{l}-0.86 \\
+0.043 \mathrm{r}\end{array}$ & $\begin{array}{l}+1.50 \\
-0.043 \mathrm{t}\end{array}$ & $\begin{array}{l}-0.021 \\
-0.013 \mathrm{r}\end{array}$ & $\begin{array}{l}+0.011 \\
+0.008 \mathrm{~T}\end{array}$ & $\begin{array}{l}-0.047 \\
-0.093 x\end{array}$ \\
\hline $\begin{aligned} a\left(\mu^{2}\right) & =1.25 \\
\mathrm{~m}_{t} & =30 \mathrm{GeV}\end{aligned}$ & $\begin{array}{l}-0.99 \\
+0.043 \mathrm{r}\end{array}$ & $\begin{array}{l}+1.60 \\
-0.043 \pi\end{array}$ & $\begin{array}{l}-0.027 \\
-0.0114\end{array}$ & $\begin{array}{l}+0.014 \\
+0.007 \tau\end{array}$ & $\begin{array}{l}-0.068 \\
-0.109\end{array}$ \\
\hline
\end{tabular}


Table II

Same as Table I but with $\Lambda^{2}=0.0 t\left(6 \mathrm{eV} \mathrm{V}^{2}\right.$

\begin{tabular}{|c|c|c|c|c|c|}
\hline Parameters & $c_{1}$ & $c_{2}$ & $c_{3}$ & $c_{5}$ & $c_{6}$ \\
\hline $\begin{aligned} a\left(u^{2}\right) & =0.75 \\
\pi_{t} & =15 \mathrm{GoV}\end{aligned}$ & $\begin{array}{l}-0.77 \\
+0.021 \tau\end{array}$ & $\begin{array}{l}+1.43 \\
-0.0218\end{array}$ & $\begin{array}{l}-0.026 \\
-0.006 \pi\end{array}$ & $\begin{array}{l}+0.013 \\
+0.004 \tau\end{array}$ & $\begin{array}{l}-0.205 \\
-0.045 .\end{array}$ \\
\hline $\begin{aligned} \alpha\left(\mu^{2}\right) & =1.00 \\
\mathrm{~m}_{\mathrm{t}} & =15 \mathrm{CeV}\end{aligned}$ & $\begin{array}{l}-0.93 \\
+0.021 \mathrm{r}\end{array}$ & $\begin{array}{l}+1.55 \\
-0.021 \tau\end{array}$ & $\begin{array}{l}-0.032 \\
-0.005 t\end{array}$ & $\left\{\begin{array}{l}+0.017 \\
+0.003 t\end{array}\right.$ & $\begin{array}{l}-0.09 \mathrm{~T} \\
-0.0 .55 \mathrm{\tau}\end{array}$ \\
\hline $\begin{aligned} a\left(\mu^{2}\right) & =1.25 \\
\pi_{5} & =15 \mathrm{GeV}\end{aligned}$ & $\begin{array}{l}-1.06 \\
+0.021 \tau\end{array}$ & $\begin{array}{l}+1.65 \\
-0.021 \mathrm{~T}\end{array}$ & $\begin{array}{l}-0.037 \\
=0.003 x\end{array}$ & $\begin{array}{l}+0.020 \\
+0.002 \tau\end{array}$ & $\begin{array}{l}-0.128 \\
-0.065 \tau\end{array}$ \\
\hline $\begin{aligned} \alpha\left(\mu^{2}\right) & =0.75 \\
m_{c} & =30 \mathrm{Gov}\end{aligned}$ & $\begin{array}{l}-0.76 \\
+0.026 \tau\end{array}$ & $\begin{array}{l}+1.42 \\
-0 . .^{-u \tau}\end{array}$ & $\begin{array}{l}-0.025 \\
-0.008 \tau\end{array}$ & $\begin{array}{l}+0.013 \\
+0.0051\end{array}$ & $\begin{array}{l}=0.065 \\
=0.060 \tau\end{array}$ \\
\hline $\begin{aligned} \alpha\left(\nu^{2}\right) & =1.00 \\
m_{t} & =30 \mathrm{GeV}\end{aligned}$ & $\begin{array}{l}-0.92 \\
+0.0277\end{array}$ & $\begin{array}{l}+1.54 \\
-0.027 \tau\end{array}$ & $\begin{array}{l}-0.032 \\
-0.006 x\end{array}$ & $\left\{\begin{array}{l}+0.017 \\
+0.004 \tau\end{array}\right.$ & $\begin{array}{l}-0.097 \\
-0.075 \tau\end{array}$ \\
\hline $\begin{aligned} \alpha\left(u^{2}\right) & =1.25 \\
\mathrm{~m}_{t} & =30 \mathrm{GeV}\end{aligned}$ & $\begin{array}{l}=1.05 \\
=0.027 \mathrm{~T}\end{array}$ & $\begin{array}{l}+1.65 \\
=0.027 \tau\end{array}$ & $\begin{array}{l}-0.037 \\
-0.004 \pi\end{array}$ & $\begin{array}{l}+0.020 \\
+0.003 \tau\end{array}$ & $\begin{array}{l}-0.127 \\
-0.088 \tau\end{array}$ \\
\hline
\end{tabular}

of "Ponguid" operators and thete mixing makts lite le numerical diffecence for the coefflciensf of $Q_{1}$ ind $Q_{2}$. Slightly mate impartant in comparison with earlier work is raking into account not only the heavy H-boson, but each heavy quark successively in compucing the lenting lag QCD effects. As a result the earlier $\left[a\left(y_{W}^{2}\right) / \alpha\left(u^{2}\right)\right]^{\text {a(t) }}$ is replaced by

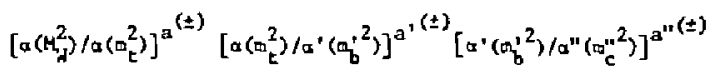

$$
\begin{aligned}
& \text { - }\left[a^{\prime \prime}\left(a_{c}^{\prime \prime 2}\right) / a^{\prime \prime}\left(\mu^{2}\right)\right]^{a^{\prime \prime \prime}( \pm)} \text {, }
\end{aligned}
$$

even LE all "Penguin" effects are neglected. Numbrically the coefficient of $q_{2}-q_{2}$ is: enhanced by a cuecor of 2 en 3 and that of $q_{2}+q_{1}$ suppressed by 0.6 to 0.7 for out choles of masses. In agreenent with all earller results this is in the correct direction, but much too small to explats the high degree of accutacy of the $\Delta I=1 / 2$ rule in nonleptante decays of strange particles.

The "Penguin" torms $Q_{3}, Q_{5}$ and $Q_{6}$ transform as purely $I=1 / 2$ on the ather hand. Tables $I$ and $I I$ indlcate that thet $T$ coeffictents are sajaller than those of $Q_{1}$ and $q_{2}$, rypically by an order of magnitude for $\eta_{6}$. However, atguments can be made chac the $(V-A) \otimes(V+A)$ structure of $Q_{b}$ may lead co enhancad matrix elements, 30 by one order of magnicude or rore, for the ronleptanic decays of haons and hypurous.

As already noted, through strong interaction effocts each operator in the effective llamlltunian has a coefficlent with an Imaginary as weIt as real part. This thaginary part. which lo each case enters through ID $x$ and is then proportional to $s_{2} c_{2}{ }^{5} 3^{\text {sind }}$, tests to $C P$ violation fit decay arolitudes. 
When $s_{2} c_{2} s_{3}$ sind $\neq 0$ and $C P$ is violated, an Inspection of the coefficients of the operators $Q_{1}$ and $Q_{2}$ timediately shows that the ratio of cheir lmaginary to real parts is $\sim 10^{-2}{ }_{5} \mathrm{C}_{2} \mathrm{~s}_{3} \mathrm{sinb}$. This is not true for the Penguin-type operators $Q_{3}, Q_{5}$ and $Q_{6}$ where the corren-

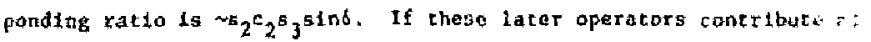
all signiftcantly to $k^{\circ}$ desay, clearly thay will y leld the Largesi $C$ P volating effects in these amp1ltudes. 16 Recal in particular that the matrix elements of $Q_{6}$ are suppogad to be espectally large and Important in decays like $k^{\circ}-\pi \pi$. This is in addition to CP violating effects which oecur in the kaon mass matrix in the $s i x-$ quark thodel, These latter CP violating effects are consldered in the follown chapter.
CHRPTER III

EFFECTIVE HAMILTONIAN FOR $\mathrm{K}^{\mathrm{D}}-\overline{\mathrm{K}}^{\circ}$ MIXING

IN THE SIX-QUARK MODEI ${ }^{31}$

The $x^{\circ}+\bar{K}^{0}$ mass matrix has played an importatit role la particle Ihysics over the past decade. The small ralue of the real part of the off diagonal elemencs fourd an explatation in the CKM mechantsal" which confeccured the extscence of a fourth quaxk flavot (charm). Later calcutacions of tife magnitude of these matrik elements Ied to a quantitatlve estimate for the charm quark tast. ${ }^{32}$ While these four-quark mode? computations tere originally done without strong interaction corrections, with the de velopment of quantur chromodynamics (QCD) the short distance wficces due to strong interation were soon compute ${ }^{33}{ }^{34}$ and tound to change the answer rather litcle.

Hith the standard phase conventinns (see Chapter IV) an fuagninary part of the of diagonal kaon mass matrix elements is an expression of $C P$ rolation and leads to the kann eigenstates $K_{L}^{0}$ and $K_{S}^{0}$ not being $C P$ elgenstates. With four quark flavors there is no lmaginary part ${ }^{9}$ but, as was mentioned in the fntroduction, the six-quark model has a phase ith the heavy quark couplings. to the weak vector bosons uhich lead to CP violation and an imaginary part to the mass matrix. In thls chapter the QCD corrections to the $\mathrm{K}^{\circ}-\mathrm{K}^{\circ}$ mass matrix are $e$ :Iculated in the alxquark Kobayashi-Maskawa 10 model.

1. Derivation of the Effect Ive Haritgonian for $k^{\circ}-\bar{x}^{\circ}$ Mixing

Using the crigonometric identities 


$$
\begin{aligned}
c_{3}^{2} c_{1}^{2} & =c_{2}^{2}\left(c_{1} c_{2} c_{3}-s_{2} s_{3} e^{-1 \delta}\right)^{2}+s_{2}^{2}\left(c_{1} s_{2} c_{3}+c_{2} s_{3} \mathrm{e}^{-1 \delta}\right)^{2} \\
& +2 s_{2} c_{2}\left(c_{1} c_{2} c_{3}-s_{2} s_{3} \mathrm{e}^{-1 \delta}\right)\left(c_{1} s_{2} c_{3}+c_{2} s_{3} \mathrm{e}^{-1 \delta}\right),
\end{aligned}
$$

$\mathrm{c}_{1} \mathrm{c}_{2} \mathrm{c}_{3}\left(\mathrm{c}_{1} \mathrm{c}_{2} \mathrm{c}_{3}-\mathrm{s}_{2} \mathrm{~s}_{3} \mathrm{e}^{-\mathrm{i} \delta}\right)=\mathrm{c}_{2}^{2}\left(\mathrm{c}_{1} \mathrm{c}_{2} \mathrm{c}_{3}-\mathrm{s}_{2} \mathrm{~s}_{3} \mathrm{e}^{-1 \delta}\right)^{2}$

$+s_{2} c_{2}\left(c_{1} c_{2} c_{3}-s_{2} s_{3} e^{-1 \delta}\right)\left(c_{1} s_{2} c_{3}+c_{2} s_{3} e^{-i \delta}\right)$

and

$$
\begin{aligned}
& c_{1} s_{2} c_{3}\left(c_{1} s_{2} c_{3}+c_{2} s_{3} e^{-1 \delta}\right)=s_{2}^{2}\left(c_{1} s_{2} c_{3}+c_{2} s_{3} e^{-1 \delta}\right)^{2} \\
& +s_{2} c_{2}\left(c_{1} c_{2} c_{3}-s_{2} s_{3} e^{-i \delta}\right)\left(c_{1} s_{2} c_{3}+c_{2} s_{3} e^{-i \delta}\right)
\end{aligned}
$$

the e.fective Hamiltonian densicy, which contributes to $k^{\circ}-\bar{K}^{\circ}$ mixing in the six-quark model, can be wricten uniquely as

$$
\begin{aligned}
\mathscr{P} P_{\text {eff }}^{\mid \Delta S t=2} & =s_{1}^{2} c_{2}^{2}\left(c_{1} c_{2} c_{3}-s_{2} s_{3} e^{-1 \delta}\right)^{2} \cdot \mathscr{F}_{1} \\
& +s_{1}^{2} s_{2}^{2}\left(c_{1} s_{2} c_{3}+c_{2} s_{3} e^{-1 \delta}\right)^{2} \mathscr{F}_{2} \\
& +2 s_{1}^{2} s_{2} c_{2}\left(c_{1} c_{2} c_{3}-s_{2} s_{3} \mathrm{e}^{-1 \delta}\right)\left(c_{1} s_{2} c_{3}+c_{2} s_{3} \mathrm{e}^{-1 \delta}\right) \mathscr{F}_{3} \div \text { h.c. }
\end{aligned}
$$

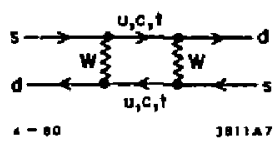

F1g. 7. Box dLagram Biving a $\mathrm{X}^{\mathrm{O}}-\overline{\mathrm{K}}^{\mathrm{O}}$ cransition.

In Eqs. (3.1) and (3.2), $s_{1}=\sin \theta_{1}, c_{1}=\cos \theta_{1}, 1 \in(1,2,3)$. The Cabibbotype angles $\theta_{1}, \theta_{2}, \theta_{3}$ and the phase $\delta$ are defined $1 n$ Eq. (1.12) of Chapter $I$ as well as in Eqs. (2.1) of Chapter II. The components, $\mathscr{H}_{1}$, $\mathscr{H}_{2}$, and $\mathscr{F}_{3}$ of the cotnplete Hamiltonian have relatively complicated expressions in terms of time ordered products of four $w$ charged currents contracted with w-boson flelds corresponding in the free-quark model to forwing the box diagram, shown 1n Ftg. 7, with virtual w-bosons and quarks in the lnnn. In the absence of strong Interactions treating the $W$-boson as very heavy and keeping only leading contribution in $1 / M_{\psi}^{2}$ 
yieldis the following expressions for $, \mathscr{P}_{1}, \mathscr{F}_{2}^{*}$, and, $\mathscr{W}_{3}$

$$
\mathscr{W}_{1}(0)=\frac{1 G_{F}^{2}}{4} \int d^{4} x\left[\mathrm { T } \left\{\left(\bar{s}_{\alpha}(x) y_{\mu}\left(1-\gamma_{5}\right) u_{\alpha}(x)\right)\left(\bar{u}_{B}(x) y^{11}\left(1-\gamma_{5}\right) d_{B}(x)\right)\right.\right.
$$

$\left.\times\left(\vec{u}_{\lambda}(0) Y^{v}\left(1-\gamma_{S}\right) u_{\lambda}(0)\right)\left(\vec{u}_{\delta}(0) \gamma_{v}\left(1-\gamma_{S}\right) d_{\delta}(0)\right)\right\}-2 T\left\{\left(\bar{s}_{\alpha}(x) \gamma_{u}\left(1-\gamma_{S}\right) u_{\alpha}(x)\right)\right.$

$\left.\times\left(\vec{u}_{B}(x) \gamma^{\mu}\left(1-\gamma_{5}\right) d_{\beta}(x)\right)\left(\vec{\varepsilon}_{\lambda}(0) \gamma_{\nu}\left(1-\gamma_{5}\right) c_{\lambda}(0)\right)\left(\bar{\varepsilon}_{\delta}(0) \gamma^{\nu}\left(1-\gamma_{5}\right) d_{\delta}(0)\right)\right\}$

$+I\left\{\left(\bar{s}_{\alpha}(x) \gamma_{\mu}\left(1-\gamma_{5}\right) c_{a}(x)\right)\left(\bar{c}_{B}(x) \gamma^{\mu}\left(1-\gamma_{5}\right) d_{B}(x)\right)\left(\bar{s}_{\lambda}(0) \gamma_{\psi}\left(l-\gamma_{5}\right) c_{\lambda}(0)\right)\right.$

$\left.\times\left(\bar{c}_{\delta}(0) \gamma^{\nu}\left(1-\gamma_{5}\right) d_{\delta}(0)\right)\right\}-2 T\left\{\left(\bar{s}_{\alpha}(x) Y_{\nu}\left(1-\gamma_{5}\right) u_{\alpha}(x)\right)\left(\bar{c}_{\beta}(x) \gamma^{\mu}\left(1-\gamma_{5}\right) d_{\beta}(x)\right)\right.$

$\left.\left.\left.\times\left(\bar{s}_{\lambda}(0) \gamma^{\nu}\left(1-\gamma_{5}\right) c_{\lambda}(0)\right)\left(\bar{u}_{s}, 0\right) \gamma_{\nu}\left(1-\gamma_{5}\right) d_{\delta}(0)\right)\right\}\right] \quad$,

$$
-x_{2}(0)=\frac{ \pm G_{F}^{2}}{4} \int d^{4} x\left[T \left\{\left(\bar{s}_{\alpha}(x) y_{\mu}\left(1-y_{S}\right) u_{\alpha}(x)\right)\left(\vec{u}_{B}(x) \gamma^{H}\left(1-y_{S}\right) d_{\beta}(x)\right)\right.\right.
$$

$\left.\times\left(\bar{s}_{\lambda}(0) \gamma^{y}\left(1-\gamma_{5}\right) u_{\lambda}(0)\right)\left(\bar{u}_{\delta}(0) \gamma_{v}\left(1-\gamma_{5}\right) d_{\delta}(0)\right)\right\}-2 T\left\{\left(\bar{g}_{a}(x) \gamma_{H}\left(1-\gamma_{5}\right) u_{\alpha}(x)\right)\right.$

$\left.\times\left(\bar{u}_{\beta}(x) \gamma^{\mu}\left(1-\gamma_{5}\right) d_{\beta}(x)\right)\left(\bar{s}_{\lambda}(0) \gamma_{\nu}\left(1-\gamma_{5}\right) t_{\lambda}(0)\right)\left(\bar{E}_{\delta}(0) \gamma^{\nu}\left(1-\gamma_{S}\right) d_{\delta}(0)\right)\right\}$

$+T\left\{\left(\bar{s}_{a}(x) y_{\mu}\left(1-\gamma_{5}\right) t_{\alpha}(x)\right)\left(\bar{t}_{B}(x) \gamma^{\mu}\left(1-\gamma_{5}\right) d_{B}(x)\right)\left(\bar{s}_{\lambda}(0) Y_{v}\left(1-\gamma_{5}\right) c_{\lambda}(0)\right)\right.$

$\left.\times\left(\bar{e}_{\delta}(0) \gamma^{v}\left(1-\gamma_{5}\right) d_{\delta}(0)\right)\right\}-2 T\left\{\left(\bar{r}_{\alpha}(x) \gamma_{u}\left(1-\gamma_{5}\right) u_{\alpha}(x)\right)\left(\tilde{t}_{\beta}(x) \gamma^{\nu}\left(1-\gamma_{5}\right) d_{\beta}(x)\right)\right.$

$\left.\left.\times\left(\bar{s}_{\lambda} i 0\right) \gamma_{v}\left(1-\gamma_{5}\right) c_{\lambda}(0)\right)\left(\vec{u}_{\delta}(0) \gamma^{v}\left(1-\gamma_{5}\right) d_{\delta}(0)\right)_{j}\right] \quad$ (3.3b)

and $\psi_{3}(0)=\frac{1 G^{2}}{4} \int d^{u} x\left[T\left\{\left(\bar{s}_{a}(x) \gamma_{u}\left(1-\gamma_{5}\right) u_{\alpha}(x)\right)\left(\bar{u}_{B}(x) \psi^{\mu}\left(1-\gamma_{5}\right) d d_{\beta}(x)\right)\right.\right.$

$\left.\times\left(\bar{s}_{\lambda}(0) \gamma^{v}\left(1-\gamma_{5}\right) u_{\lambda}(0)\right)\left(\bar{u}_{\delta}(0) \gamma^{v}\left(1-\gamma_{5}\right) d_{\delta}(0)\right)\right\}-I\left\{\left(\bar{s}_{a}(x) \gamma_{\mu}\left(1-\gamma_{5}\right) u_{a}(x)\right)\right.$

$\left.\times\left(\bar{u}_{B}(x) \gamma^{W}\left(1-\gamma_{5}\right) d_{B}(x)\right)\left(\bar{s}_{\lambda}(0) \gamma_{y}\left(1-\gamma_{5}\right) c_{\lambda}(0)\right)\left(\bar{c}_{\delta}(0) \gamma^{\nu}\left(1-\gamma_{5}\right) d_{B}(0)\right)\right\}$

- If( $\left(\bar{s}_{\bar{L}}(x) \gamma_{\mu}\left(1-\gamma_{5} ; u_{\square}(x)\right)\left(\bar{u}_{B}(x) \gamma^{\mu}\left(1-\gamma_{5}\right) d_{B}(x)\right)\left(\bar{s}_{\lambda}(0) \gamma_{\nu}\left(1-\gamma_{5}\right) t_{\lambda}(0)\right)\right.$

$\left.\times\left(\bar{c}_{\delta}(0) \gamma^{\nu}\left(1-\gamma_{S}\right) d_{\delta}(0)\right)\right\}-T\left\{\left(\bar{s}_{a}(x) \gamma_{\mu}\left(1-\gamma_{S}\right) u_{a}(x)\right)\left(\bar{c}_{B}(x) \gamma^{\mu}\left(1-\gamma_{S}\right) d_{g}(x)\right)\right.$

$\times\left(\bar{s}_{\lambda}(0) \gamma^{v}\left(1-\gamma_{5}\right) c_{\lambda}(0)\right)\left(\bar{u}_{\delta}\left(0 \gamma_{\nu}\left(1-\gamma_{5}\right) d_{d}(0)\right)\right\}-T\left\{\left(\bar{s}_{a}(x) \gamma_{y}\left(1-\gamma_{5}\right) u_{a}(x)\right)\right.$

$\left.\times\left(\bar{t}_{\beta}(x) \gamma^{\mu}\left(1-\gamma_{5}\right) d_{B}(x)\right)\left(\bar{s}_{\lambda}(0) \gamma_{v}\left(1-\gamma_{5}\right) \tau_{\lambda}(0)\right)\left(\bar{u}_{b}(0) \gamma^{v}\left(1-\gamma_{5}\right) d_{d}(0)\right)\right\}$

$+T\left\{\left(\bar{s}_{\alpha}(x) \gamma_{\nu}\left(1-\gamma_{5}\right) c_{\alpha}(x)\right)\left(\bar{t}_{B}(x) \gamma^{\nu}\left(1-\gamma_{5}\right) d_{B}(x)\right)\left(\bar{s}_{\lambda}(0) \gamma^{\nu}\left(1-\gamma_{5}\right) c_{\lambda}(0)\right\}\right.$

$\left.\times\left(\bar{c}_{\delta}(0) \gamma_{\nu}\left(1-\gamma_{5}\right) d_{\delta}(0)\right)\right\}+T\left\{\left(\bar{s}_{\alpha}(x) \gamma_{H}\left(1-\gamma_{S}\right) c_{\alpha}(x)\right)\left(\bar{c}_{B}(x) \gamma^{\mu}\left(1-\gamma_{5}\right) d_{B}(x)\right)\right.$

$\left.\left.\times\left(\bar{s}_{\lambda}(0)_{\gamma_{-}}\left(1-\gamma_{5}\right) t_{A}(0)\right)\left(\overrightarrow{\mathrm{r}}_{\delta}(0) \gamma^{0}\left(1-\gamma_{5}\right) d_{d}(D)\right)\right\}\right]$

a, $B, A$ and 8 are color indices which are sured over $\{1,2,3\}$ when repeated. Normal ordering of the loça 4-quark operators is understood. It is conventent to decompose the se operators into pleces that w1li not mix under renormallzation when the strong interactions are intruduced. We write for $j c(1,2,3)$ (1n the sbsence of strong interactfons)

$$
\mathscr{W}_{j}=\frac{\mathrm{c}_{\mathrm{F}}^{2}}{16}\left[\mathscr{m}_{\mathrm{j}}^{(++)}+\mathscr{W}_{\mathrm{j}}^{(+-)}+\mathscr{H}_{\mathrm{j}}^{(+)}+\mathscr{W}_{\mathrm{j}}^{(--)}\right] \text {. }
$$




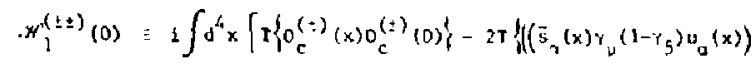

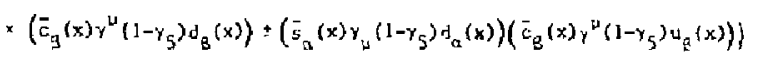

$$
\begin{aligned}
& \times\left(\left(\bar{s}_{\lambda}(0) r^{v}\left(1-r_{g}\right)_{c_{d}}(0)\right)\left(\tilde{u}_{j}(0) r_{v}\left(1-r_{5}\right) d d_{g}(0)\right)\right. \\
& \left.\left.\left. \pm\left(\bar{s}_{\lambda}(0) \cdot{ }^{\nu}\left(1-\gamma_{5}\right) d,(0)\right)\left(\tilde{u}_{\delta}(0) \gamma_{v}\left(1-\gamma_{5}\right) e_{\delta}(0)\right)\right)\right\}\right] \\
& \mathscr{F}_{2}^{(t+2)}(0) \equiv ! \int d^{4} x\left[r\left\{0_{t}^{(*)}(x) 0_{t}^{(*)}(0)\right\}-2 T\left\{\left(\left(\bar{s}_{x}(x) \gamma_{\mu}\left\{1-\gamma_{5}\right) u_{\alpha}(x)\right)\right.\right.\right. \\
& \left.\times\left(\bar{t}_{B}(x) \gamma^{\mu}\left(1-\gamma_{g}\right) d d_{B}(x)\right)+\left(\bar{s}_{a}(x) \gamma_{u}\left(1-\gamma_{g}\right) d_{g}(x)\right)\left(\bar{e}_{B}(x) \gamma^{H}\left(1-\gamma_{g}\right) u_{B}(x)\right)\right) \\
& \times\left[\left(\bar{s}_{\lambda}(0) r_{u}\left(1-r_{5}\right) r_{\lambda}(0)\right)\left(\bar{u}_{\delta}(0) Y^{u}\left(1-r_{5}\right) d_{\delta}(0)\right)\right.
\end{aligned}
$$

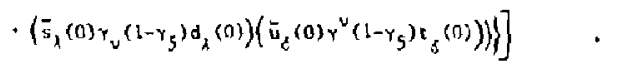

$$
\begin{aligned}
& \text { and }
\end{aligned}
$$

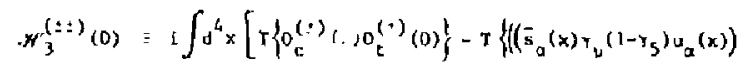

$$
\begin{aligned}
& \left.*\left(\bar{c}_{\mu}(x) \gamma^{\mu}\left(1-\gamma_{5}\right) d_{B}(x)\right):\left(\bar{s}_{a}(x) \gamma_{k}\left(1-\gamma_{5}\right) d_{a}(x)\right)\left(\bar{c}_{B}(x){ }^{p}\left(1-\gamma_{5}\right) u_{B}(x)\right)\right)
\end{aligned}
$$

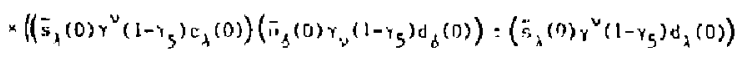

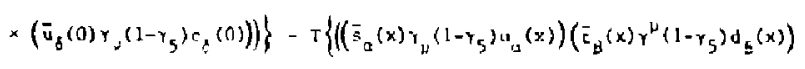

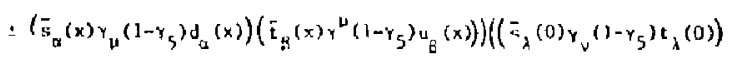

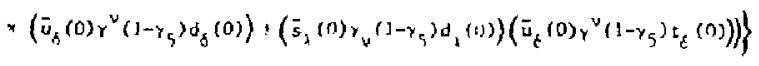

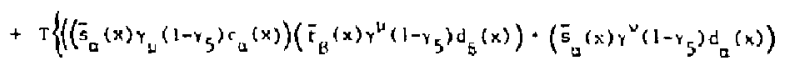

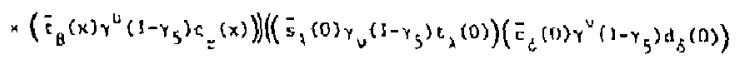

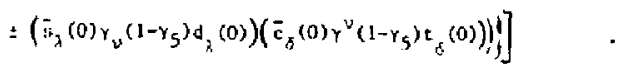

$o_{c}^{(t)}$ and $o_{t}^{(+)}$are deffined in Eq- (2.) of Chapter II.

Now Introduce strong Inceractlons in the form of quantur chromodynamics (gca). The pleces of the effective Homittenian , $x_{f}$ defined by Eq. (3.2) are modfied from this, free fletd expresstons given in Eqs. (3.3) and (3.4). Treating the 4 -boson as heavy in the presence of strong inceractions yleids the following expregsion

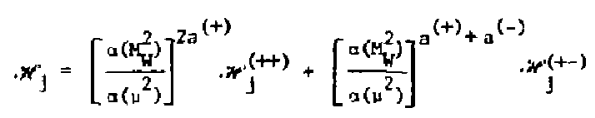

$$
\begin{aligned}
& +\left[\frac{a\left(M_{w}^{2}\right)}{a\left(u^{2}\right)}\right]^{a^{(-)}+a^{(+)}} w_{j}^{(-)}+\left[\frac{a\left(m_{w}^{2}\right)}{g\left(u^{2}\right)}\right]^{2 x} \cdot w_{j}^{(-)} \cdot
\end{aligned}
$$

In the leading logartchaic approximation. A Is che strong inceraction ine strueture constant, and the renomaltzation point. The matrix elements of the $\mathscr{W}_{j}$ are to be evaluated to all orders (alnce perturbaction theory is probably not valtd) in the six-quark theory of strong interactions using the $\overline{\text { MS }}$ subtraction gsheme. Finally if ${ }^{(+)}$ax $6 / 21$ and $a^{(-)}=$ $-12 / 21$. The derivation of $E q$. (3.6) is very stailax to the rewoval of the H-boson fleld from the effert ve Hamiltonian for $\Delta S=1$ weak nonleptonle decays discussed in Chapter II.

The next step Is co successively treat the r-quark, b-quark and e-quirk as heavy and remove thef flelds from explicitly appearing in the theory. Fof $W_{i}$ this is parcleularly stmple since the $t$ and b-quark 
flatus do not pppear explectely in it. "'e effect of removing the and bequark flelds from the theory of strong interactions is to change the strong coupling $B$ and thasses $m_{11}, \ldots, m_{t}$ in the six-quark theury to $a$ corupling $\mathrm{g}^{\prime}$. and maseses $\mathrm{m}_{\mathrm{b}}^{\prime}, \ldots, \mathrm{m}_{\mathrm{b}}^{\prime}$ in an effecsive 5-quark ehicary and stren to a coupling $g "$ and rasses $m_{u}^{\prime \prime}, \ldots, m_{c}^{\prime \prime}$ in an effective 4 -quark theory of the strong tnteractlons. A1so the exponents $a^{(+)}\left(a^{(-)}\right)$change from 6/21 $(-12 / 21)$ to $6 / 23(-1, / 23)$ and then to $6 / 25(-12 / 25)$ as one goes from the Ex-quark thenry to the effective 5-quark theory and then to the effective i-quark theory of strong interactions, Thus the effectlve Hamiltanian denatty $\mathscr{N}_{1}$ becomss

$$
\begin{aligned}
& \mathscr{H}_{1}=\left[\frac{a\left(m_{W}^{2}\right)}{a\left(m_{t}^{2}\right)}\right]^{12 / 21}\left[\frac{a\left(m_{t}^{2}\right)}{a^{\prime}\left(m_{b}^{2}\right)}\right]^{12 / 73}\left[\frac{a^{1}\left(m_{b}^{2}\right)}{a^{2}\left(\omega^{2}\right)}\right]^{12 / 25} x_{1}^{(++)} \\
& +\left[\frac{a\left(\mu_{w}^{2}\right)}{u\left(m_{c}^{2}\right)}\right]^{-6 / 21}\left[\frac{a\left(m_{c}^{2}\right)}{2 \cdot\left(m_{b}^{\prime 2}\right)}\right]^{-6 / 23}\left[\frac{a^{\prime}\left(m_{b}^{2}\right)}{u^{\prime \prime}\left(\omega^{2}\right)}\right]^{-6 / 25} x_{2}^{(+-)} \\
& +\left[\frac{n\left(m_{b}^{2}\right)}{n\left(m_{t}^{2}\right)}\right]^{-6 / 21}\left[\frac{a\left(m_{t}^{2}\right)}{x^{\prime}\left(m_{b}^{\prime 2}\right)}\right]^{-6 / 23}\left[\frac{a^{\prime}\left(m_{b}^{\prime 2}\right)}{a^{\prime \prime}\left(v^{2}\right)}\right]^{-6 / 25} \cdot x_{1}^{(-4)}
\end{aligned}
$$

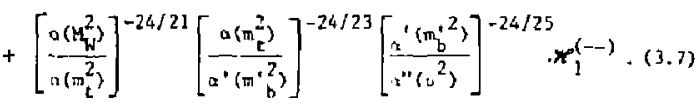

The matrix $z^{*}$ rents of the effective Hamiltongan density $x_{1}$ are row to be evaluated in an affective 4-quars theory of strong interactions. It onlv remains to treat the charm quark as heavy and renove 1 t froro explicitly appearing in $\mathscr{H}_{1}$. To leadlng order in the s-quark aass the natelx elements of $x_{j}^{( \pm t)}$ can be expanded in the following fashion

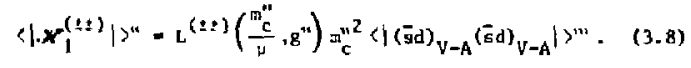

The double primed matefx elonts are evaluated in an cffective fourquark thenry of strong interactions while the triple primed matrix elements are to be evaluated in an effect lve three-quark theory of strong Interaccions with coupling $g^{\prime \prime \prime}$ and misses $m_{u}^{\prime \prime \prime}, m_{d}^{\prime \prime \prime}$ and $m_{s}^{\prime \prime \prime}$. The operator $(\bar{s} d)_{V-A}(\bar{s} d) V_{-\Lambda}$ is a color symetric four femion operator with the usual anomalous dimension

$$
r^{\prime \prime \prime(+)}\left(\varepsilon^{\prime \prime \prime}\right)=\frac{g^{m 2}}{4 \pi^{2}}+r \cdot\left(g^{\prime \prime \prime}\right)
$$

The mass parsineter fi"c depends on the renormalization polnt $u$ and 1 ts anomalous dimension is

$$
r_{r}^{\prime \prime}\left(g^{\prime \prime}\right)=\frac{g^{\prime \prime 2}}{2 \pi^{2}}+r\left(R^{* n^{4}}\right)
$$

The compontents $\boldsymbol{X}_{1}^{(+)}, \boldsymbol{x}_{1}^{(+-)}, \boldsymbol{X}_{1}^{(+)}$and, $\boldsymbol{x}_{1}^{(--)}$are composed of a sum of thm nxdered products of two local four-quark aperaenes with color indices: respectively swmetrlzed in both operators, symetrized in the first operutor and antisymetrlzed in the second operator, antisymectized in the first operator and symatrlzed in the second operator and finally antisytuectzed in both operators. They have the fantllar anomalous dimensions, $5.6 \mathrm{~g}^{\prime 2} / 2 \pi^{2}+r \cdot\left(\mathrm{g}^{114}\right),-\mathrm{g}^{\prime 2} / 6 \pi^{2}+r\left(\mathrm{~g}^{+4}\right),-\mathrm{g}^{\prime \prime 2} / 4 \pi^{2}+f\left(\mathrm{~g}^{\prime \prime 4}\right)$. and $-g^{\prime 2} / \pi^{2}+r\left(g^{m 4}\right)$ respect Ively. It follows that the H1lson coefflclerrs $L^{( \pm)}\left(m_{c}^{\prime \prime} / \mu, g^{\prime \prime}\right)$ obey the renorallation group equatlons 
$\left(\mu \frac{\partial}{\partial \mu}+g^{\prime \prime \prime}\left(g^{\prime 1}\right) \frac{\partial}{\partial g^{\prime \prime}}+\gamma_{c}^{\prime \prime}\left(g^{\prime \prime}\right) m_{c}^{\prime \prime} \frac{\partial}{\partial m_{c}^{\prime \prime}}-\frac{g^{\prime \prime 2}}{2 \pi^{2}}-\frac{g^{\prime \prime 2}}{6 \pi^{2}}\right) L(+)\left(\frac{\pi_{c}^{\prime \prime}}{\nu}, g^{\prime \prime}\right)=0 \quad$.

(3.11a)

$\left(\mu \frac{\partial}{\partial \mu}+g^{\prime \prime}\left(g^{\prime \prime}\right) \frac{\partial}{\partial g^{\prime \prime}}+\gamma_{c}^{\prime \prime}\left(g^{\prime \prime}\right) m_{c}^{\prime \prime} \frac{\partial}{\partial m_{c}^{\prime \prime}}-\frac{5 g^{\prime \prime 2}}{4 \pi^{2}}-\frac{g^{\prime \prime 2}}{4 \pi^{2}}\right) L^{(+-)}\left(\frac{m_{c}^{\prime \prime}}{\mu}, g^{\prime \prime}\right)=0$,

(3.11b)

$\left(\nu \frac{\partial}{\partial \mu}+B^{\prime \prime}\left(B^{\prime \prime}\right) \frac{\partial}{\partial g^{\prime \prime}}+Y_{c}^{\prime \prime}\left(g^{\prime \prime}\right) m_{c}^{\prime \prime} \frac{\partial}{\partial m_{c}^{\prime \prime}}-\frac{5 g^{\prime \prime 2}}{4 \pi^{2}}-\frac{g^{\prime \prime \prime 2}}{4 \pi^{2}}\right) L^{(-)}\left(\frac{m_{c}^{\prime \prime}}{\mu}, g^{\prime \prime}\right)=0$,

(3.1le)

$\left(\mu \frac{\partial}{\partial \mu}+\beta^{\prime \prime}\left(g^{\prime \prime}\right) \frac{\partial}{\partial g^{\prime \prime}}+\gamma_{c}^{\prime \prime}\left(g^{\prime \prime}\right) m_{c}^{t \prime} \frac{\partial}{\partial m_{c}^{\pi}}-\frac{2 g^{\prime \prime 2}}{\pi^{2}}-\frac{{g^{\prime \prime 2}}^{2}}{4 \pi^{2}}\right) L^{(--)}\left(\frac{m_{c}^{\prime \prime}}{\mu}, g^{\prime \prime}\right)=0$.

These may be solved in the standard fashion, fntroductig a running coupling constant $\overline{g^{\prime \prime}}\left(y, g^{\prime \prime}\right)$ defined by

$$
\text { en } y=\int_{g^{\prime \prime}}^{\overline{g^{\prime \prime}}\left(y, g^{\prime \prime}\right)} d x \frac{1-\gamma_{c}^{\prime \prime}(x)}{\beta^{\prime \prime}(x)}, \overline{g^{\prime \prime}}\left(1, g^{\prime \prime}\right)=g^{\prime \prime} \text {. }
$$

and noting that the coefficients $L^{( \pm \pm)}\left(l, \mathrm{~g}^{\text {"I }}\left(\mathrm{a}_{c} / \mu, \mathrm{g}^{\prime \prime}\right)\right)$ may be replaced by thetr free field values $L^{( \pm \pm)}(1,0)$ since the running fine structure constant is caken as small at the scale of the charm quark mass and because no large logarichess can be generated from higher order Q QCr loop incegrals when $m_{c}^{\prime \prime \prime} / \mu=1$. A straightforward conputat lon y1tids

and

$$
\begin{aligned}
& L^{(t+)}(1,0)=-\frac{1}{T^{2}}\left[\frac{3}{2}\right] \\
& L^{(t-)}(1,0)=L^{(-+)}(1,0)=-\frac{1}{\pi^{2}}\left[-\frac{1}{2}\right] .
\end{aligned}
$$

$$
L^{(\rightarrow)}(1,0)=-\frac{1}{\pi^{2}}\left[\frac{1}{2}\right]
$$

The factors in the squate brackets stem from color surmations. Solving the reromalizacton group Eqs. (3.11) using the leading logariehmic approximation then gives

$$
\text { J. }{ }^{(++)}\left(\frac{m_{c}^{\prime \prime}}{\mu} \cdot g\right)=-\frac{1}{\pi^{2}}\left[\frac{a^{\prime \prime \prime}\left(\mu^{2}\right)}{a^{\prime \prime}\left(m_{c}^{\prime 2}\right)}\right]^{12 / 25}\left[\frac{\alpha^{\prime \prime}\left(m^{\prime \prime 2}\right)}{a^{\prime \prime \prime}\left(\mu^{2}\right)}\right]^{6 / 27}\left[\frac{a^{\prime \prime}\left(m^{\prime \prime 2}\right)}{a^{\prime \prime}\left(\mu^{2}\right)}\right]^{24 / 25}\left[\frac{3}{2}\right] \text {. }
$$

$$
\begin{aligned}
& L^{(+)}\left(\frac{m_{c}^{n+1}}{L}, g\right)=L^{(+)}\left(\frac{m_{c}^{n \prime}}{\mu}, g\right) \\
& =-\frac{1}{\pi^{2}}\left[\frac{a^{\prime \prime}\left(u^{2}\right)}{a^{\prime \prime}\left(a_{c}^{\prime 2}\right)}\right]^{-6 / 25}\left[\frac{a^{\prime \prime}\left(m^{\prime 2}{ }^{2}\right)}{r^{\prime \prime \prime}\left(\mu^{2}\right)}\right]^{5 / 2 T}\left[\frac{a^{\prime \prime}\left(a_{c}^{\prime 2}\right)}{a^{\prime \prime}\left(p^{2}\right)}\right]^{24 / 25}\left[-\frac{1}{2}\right] \text {. }
\end{aligned}
$$

and

$$
L^{(--)}\left(\frac{m^{\prime \prime}}{\mu}+8\right)=-\frac{1}{2}\left[\frac{a^{\prime \prime}\left(\mu^{2}\right)}{\alpha^{\prime \prime}\left(m_{c}^{\prime \prime 2}\right)}\right]^{-2 L^{2} / 25}\left[\frac{a^{\prime \prime}\left(m^{\prime \prime}{ }^{2}\right)}{\alpha^{\prime \prime 1}\left(u^{2}\right)}\right]^{6 / 27}\left[\frac{a^{\prime \prime}\left(m_{c}^{\prime \prime 2}\right)}{a^{\prime \prime}\left(\mu^{2}\right)}\right]^{24 / 25}\left[\frac{1}{2}\right] .
$$

Using these cesults the effective Hamiltonlan densicy, $\neq_{1}$ becomes

$$
\begin{aligned}
& x_{1}=-\frac{C_{F}^{2}}{16 r^{2}} m_{c}^{2}\left(\bar{s}_{a} r^{\mu}\left(1-r_{5}\right) d_{Q}\right)\left(\bar{s}_{B} r_{u}\left(1-\gamma_{5}\right) d_{g}\right)
\end{aligned}
$$

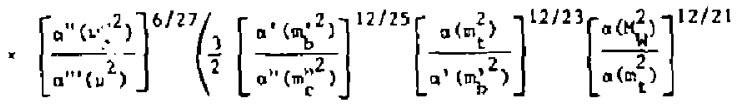

$$
\begin{aligned}
& -\left[\frac{a^{\prime}\left(m_{b}^{2}\right)^{2}}{a^{\prime \prime}\left(m_{c}^{\prime 2}\right)}\right]^{-6 / 25}\left[\frac{a\left(m_{L}^{2}\right)}{a^{\prime}\left(m_{b}^{2}\right)}\right]^{-6 / 23}\left[\frac{a\left(m_{b}^{2}\right)}{a\left(a_{t}^{2}\right)}\right]^{-6 / 21} \\
& \left.+\frac{1}{2}\left[\frac{a^{\prime}\left(m_{b}^{\prime 2}\right)}{a^{11}\left(m_{c}^{\prime 2}\right)}\right]^{-24 / 25}\left[\frac{a\left(m_{t}^{2}\right)}{a^{\prime}\left(m_{b}^{\prime 2}\right)}\right]^{-24 / 23}\left[\frac{a\left(m_{w}^{2}\right)}{a\left(m_{t}^{2}\right)}\right]^{-24 / 21}\right)
\end{aligned}
$$


where $m_{c}^{\star}$ to the running charro quark mass evaluated at $m_{c}^{\prime \prime 2}$, 1.e.,

$$
m_{c}^{*}=m_{c}^{\prime \prime}\left[\alpha_{s}^{\prime \prime}\left(m_{c}^{\prime \prime 2}\right) / a_{s}^{\prime \prime}\left(w^{2}\right)\right]^{12 / 25} \text {. }
$$

The Hamiltonian $\boldsymbol{x}_{1}$ already accurs it. the four-quark model and our results agree with some of the prowlous results ${ }^{33}$ for the QCD corrected $\mathscr{H}_{1}$, when the appropriate simplifications are tade.

The deviation of the effective Humilionian denstty $\mathscr{X}_{2}$ proceeds along gimflar 1fnes except thet al tealdy at the step of $r 6$ joving the $t$-quark fleld from explicitly appearing each of the $\cdot x_{2}^{(+)}, x_{2}^{(+)}, x_{2}^{(-)}$and $-x_{2}^{(--)}$collapges to a wilson coeffletent times $m_{5}^{2}\left(\bar{s}_{a} \gamma_{L}\left(1-r_{5}\right) d_{a}\right) x$ $\left(\bar{s}_{B} Y^{\mu}\left(1-\gamma_{5}\right) d_{B}\right)$ to leading order tn the t-quatk mass. From that point on the successive sceps are garked by renormalfzation of this latter color findex symatric four-fermion operator. The final resule is

$$
\begin{aligned}
& x_{2}=-\frac{G_{E}^{2} m_{t}^{k 2}}{16 \pi^{2}}\left(\bar{s}_{\alpha} Y_{\mu}\left(1-\gamma_{5}\right) d_{a}\right)\left(\bar{s}_{B} \gamma^{\mu}\left(1-\gamma_{5}\right) d_{B} .\right. \\
& \times\left[\frac{a^{\prime \prime}\left(m_{c}^{\prime \prime 2}\right)}{a^{\prime \prime \prime}\left(u^{2}\right)}\right]^{6 / 27}\left[\frac{a^{+}\left(m_{b}^{2}\right)}{a^{\prime \prime}\left(m_{c}^{\prime 2}\right)}\right]^{6 / 25}\left[\frac{a\left(m_{c}^{2}\right)}{a^{\prime}\left(m_{b}^{\prime 2}\right)}\right]^{6 / 23} \\
& \times\left(\frac{3}{2}\left[\frac{\alpha\left(m_{W}^{2}\right)}{\alpha\left(n_{t}^{2}\right)}\right]^{12 / 21}-\left[\frac{\alpha\left(m_{L}^{2}\right)}{\alpha\left(m_{L}^{2}\right)}\right]^{-6 / 2 I}+\frac{1}{2}\left[\frac{\alpha\left(m_{W}^{2}\right)}{\left.a^{2} \cdot m_{\tau}^{2}\right)}\right]^{-24 / 21}\right) \text {, }
\end{aligned}
$$

where $m_{t}^{*}$ is the running $t$-quark mass evaluated at $m_{t}^{2}$, f.e., $m_{c}^{*}=m_{t}\left[a\left(m_{t}^{2}\right) / \alpha\left(u^{2}\right)\right]^{12 / 21}$.

The computation of the effect: $v_{4}$ Hamlitonian densley $\boldsymbol{x}_{3}$ in the presence of strong interactions is somewhat morc complex. At cii. step of removing the t-quark from $x_{3}^{( \pm \pm)}$eight operacots are generated even with the condition of keeping only those whose matrix elements can yield a contribution of order $m_{c}^{\prime \prime 2}$ or $m l x$ under renormalization with operators whoge matrix elements can. Expanding the matrix elements of $x_{3}^{( \pm \pm)}$in zerms of matrix elements of these operators gives

$$
\left\langle\left|, x_{3}^{( \pm \pm)}\right|\right\rangle=\sum_{j=1}^{7} L_{j}^{( \pm \pm)}\left\langle\left|0_{j}^{( \pm \pm)}\right|\right\rangle^{\prime}+L_{B}^{( \pm \pm\rangle}\left\langle\left|O_{g}\right|^{\prime}\right.
$$

to 1eading order in the t-quark mass. The primed matrix elements are evaluated in an effective 5-quatk theory with strong coupling $g^{\prime}$. Six of the operators

$$
\begin{aligned}
& \left.\left.O_{1}^{( \pm \pm)}=t \int d^{4} x T\right\}_{c}^{( \pm)}(x)\left(\bar{s}_{\alpha}{ }_{d}\right)_{V-A}\left(\bar{u}_{G}{ }_{B}{ }_{B}\right)_{V-A}\right\}^{\prime} . \\
& \left.\left.O_{2}^{( \pm \pm)}=1 \int d^{4} x T\right\}_{C}^{( \pm)}(x)\left(\bar{s}_{a} d_{B}\right) V_{V-A}\left(\bar{u}_{B} u_{a}\right)_{V-A}\right\} \text {, } \\
& \left.0_{3}^{( \pm \pm)}=1 \int d^{4} \times T ; 0_{c}^{( \pm)}(x)\left(\tilde{s}_{a} d\right) v_{-A}\left[\left(\bar{u}_{B} v_{B}\right)_{V-\Lambda}+\ldots+\left(\bar{b}_{B} b_{B}\right)_{V-A}\right]\right\} \text {. }
\end{aligned}
$$

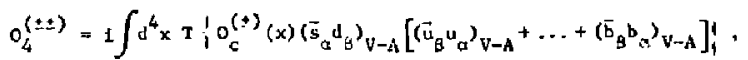

$$
\begin{aligned}
& 0_{5}^{( \pm \pm)}=i \int d^{4} x T 0_{c}^{(t)}(x)\left(\bar{s}_{\alpha} d a v_{-A}\left[\left(\bar{u}_{B} u_{B}\right)_{v+A}+\ldots+\left(\bar{b}_{B} b_{B}\right)_{v+A}\right]\right\} \text {, } \\
& o_{G}^{( \pm t)}=\left[\int d^{2} x T, o_{c}^{(s)}(x)\left(\bar{s}_{a} d_{\beta}\right)_{v-A}\left[\left(\bar{u}_{B} u_{d}\right)_{v+A}+\ldots+\left(\bar{E}_{B} b_{a}\right)_{v+A}\right]_{1}^{1},\right.
\end{aligned}
$$

orfiglate from the portion of $x_{3}^{( \pm t)}$.

$$
1 \int d^{4} \times T 0_{c}^{( \pm)}(x) 0_{c}^{(x) !}
$$


which is an integral of a time ordered product of two pieces of the effective $\Delta S=l$ weak nenleptonic HamLltonian, one containing a t-quark and the other $\Delta c^{-}$-quark. Notu that $o_{j}^{(1 \pm)}=o_{j}^{( \pm f)}$ for $j \in\{1, \ldots, 6\}$. The two additional operators needed are

$$
\begin{aligned}
& o_{z}^{( \pm x)}=1 \int d^{4} x+\left\{\left[\left(\bar{s}_{a}(x) \gamma_{\nu}\left(1-\gamma_{S}\right) \nu_{\alpha}(x)\right)\left(\bar{c}_{B}(x)_{Y}{ }^{\mu}\left(1-\gamma_{5}\right) d_{B}(x)\right)\right.\right. \\
& \left. \pm\left(\bar{s}_{a}(x) Y_{u}\left(1-y_{5}\right) d_{a}(x)\right)\left(\bar{c}_{B}(x) Y^{H}\left(1-y_{5}\right) u_{p}(x)\right)\right]
\end{aligned}
$$

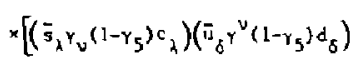

$$
\begin{aligned}
& \left.\left. \pm\left(\bar{s}_{A} \gamma_{v}\left(1-\gamma_{5}\right) A_{\lambda}\right)\left(\bar{u}_{\delta} r^{v}\left(1-\gamma_{5}\right) c_{\delta}\right)\right]\right\} \\
& p_{8}=\frac{m_{c}^{\prime 2}}{g^{\prime 2}}\left(\bar{s}_{a} \gamma^{b}\left(1-\gamma_{5}\right) d_{a}\right)\left(\bar{s}_{B} \gamma_{p}\left(1-\gamma_{5}\right) d_{B}\right) .
\end{aligned}
$$$$
\text { and }
$$

The factor of $1 / \mathrm{g}^{\prime 2}$ is inserted fnto the definition of $o_{8}$ so that to lowest order the anomalous dimension matrix $\gamma_{i j}^{( \pm t)}$ (g') has all its entries proportional to $\mathrm{g}^{2}$. If $\mathrm{o}_{\mathrm{B}}$ did not contain the Eactor of $1 / \mathrm{g}^{2}$ then the elements $\gamma_{i g}^{( \pm \pm)}\left(B^{\prime}\right)$ wadd be (eo lowest order) constants independent of $\mathrm{g}^{\prime}$ for $1 \in(1, \ldots, 7\}$. Then in solving the renormalization group equations $L_{k}^{( \pm \pm)}$vould have to be treated in a different fastion from the $L_{j}^{( \pm t)}$. J $E\{1, \ldots, 7\}$. On the other hand, with our defintion ${ }^{35}$ of $O_{8}$ it can be reosed on the same footing as all the other operators. of course in calculating its renormalfzation we must now be careful to Include the coupligg constant renomalization. The matrix elements of the operators $\mathrm{o}_{1}^{( \pm+)}$and $\mathrm{o}_{2}^{(t+)}$ cannor produce a factor of $\mathrm{m}_{\mathrm{c}}{ }^{2}$, however, they musc in principle be included since under renardalization they mix w th the operators $o_{3}^{(2 \pm)}, o_{6}^{( \pm \pm)}$, ete. whose matrik elements can produce a factor of $m_{c}^{\prime \prime 2}$. The anomalous dlmenseun matrices $y_{i j}^{( \pm+)}\left(g^{\prime}\right)$ far these eighe operators are $\mathrm{k}$ iven in Appendix $B$. The coefffetents $L_{j}^{( \pm \pm)}\left(m_{t} / \mu, g\right)$ satisfy runomalieacion proup equarions which can be solved in the stondard way. In this nolution values ate needed for the coefficienta $L_{-j}^{(!:)}\left(1, \bar{g}\left(m_{t} / 4, g\right)\right)$, where $\bar{g}$ is the running coupling in the six-quark thenry defined in Ea. (2.13) of Chapter 11 . These are found by noring that in the leading logarteheste approximation the $I_{j}^{( \pm \pm)}\left(1, \bar{g}\left(m_{t} / \mu, g\right)\right)$ can be repluced by thetr eree field values $L_{j}^{( \pm)}(1,0)$ for $\mathrm{j}$ c $\{l, \ldots, 7\}$.

$$
\begin{aligned}
& L_{1}^{( \pm \pm)}(1,0)= \pm 1 . \\
& L_{2}^{( \pm \pm)}(1,0)=1 . \\
& L_{3}^{( \pm \pm)}(1,0)=L_{4}^{( \pm \pm)}(1,0)=L_{5}^{( \pm \pm)}(1,0)=L_{6}^{( \pm \pm)}(1,0)=0,
\end{aligned}
$$

and

$$
L_{7}^{( \pm \pm)}(1,0)=-1
$$

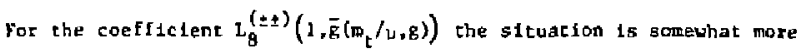
subcle since the operator $O_{8}$ contains a factor of $1 / g^{2}$. Explicit calculation gives that in the $\overline{M S}$ regularization schede

$$
\left.L_{7}^{( \pm \pm)}\left(m_{t} / \mu=1, \bar{B}\right) \propto \bar{g}^{2} \mathrm{Rn}\left(m_{t}^{2} / \mu^{2}\right)\right|_{u=m_{t}}=0 .
$$

The Iast step follows, not because the factor of $\bar{\varepsilon}^{2}$ is smat1, but rather because the logarithm vanistes at $\mu=w_{t}$. The final aim is to derive an effective Hamiltonian Independent of the heavy $W$-boson, t-quark. b-hiuark and e-quark fields. To do this the b-quark and c-quark wust still be constdered as heavy and removed from explicitly appearing in the theory. Remova" af the b-quatk is sirilar to the previous step. There are still 
etght operators whose renoznalization is charactertzed by the anowalous dtmension matrices $y^{\prime \prime \pm t}\left(g^{\prime \prime}\right)$ given in Appendix $B$. Finally at the step of removing the charm quark only one operator $m_{c}^{\prime \prime 2}\left(\bar{s}_{\alpha} y_{\nu}\left(1-r_{5}\right) d \alpha\right)$ * $\left(\bar{s}_{\beta} \gamma^{\mu}\left(1-\gamma_{5}\right) d_{\beta}\right)$ appears and tes anomalous dimenston follows from mass renomialization and the renormalization of the color symetric local four-fernfor operator $(\bar{s} d) v_{-A}(\bar{s} d) v_{-A}$. ThIs program for deriving the effective Hamiltonian $x_{3}$ in the presence of streng interactions is a straightforwatd generalization of that used fn Chapter II ca derive the effective Hamiltonian for weok nomleptonic decays, Its complexity Is such that, unlike the case of $\mathscr{X}_{1}$ and $\mathscr{X}_{3}$, we cannot write a simple analytic expreseion for $\boldsymbol{\pi}_{3}$. However thete are some further appraximattons, beyond the leading logartitmic approx1mation, which make the Cinivaction of a simple analytic expression for $\boldsymbol{x}_{3}$ passtbla. As can be seen from Eqs. (3.22) the pperators $0_{3}^{( \pm)}, \ldots, 0_{6}^{( \pm)}$are induced through strong Interactions and thus their contribution Is less importanc than $0_{7}^{( \pm \pm)}$which has a non-zera coeffictent even in the abitence of the strong interactions. It follows since $o_{1}$ and $a_{2}$ do not mix directly with $o_{7}$ and $r_{8}$ that to a goad approximation, at the stage of removing the $t-$ quark, the set of eight operators can be truncated to the tuo operators $o_{7}^{( \pm \pm)}$and $o_{g}$. These two operators then have the $2 \times 2$ anomalous dfmension matrices:

$$
\begin{aligned}
& \gamma^{\prime(+)}\left(g^{\prime}\right)=\frac{g^{\prime 2}}{8 \pi^{2}}\left(\begin{array}{cc}
4 & -24 \\
0 & 7 / 3
\end{array}\right)+\rho\left(g^{\prime 4}\right) \\
& \gamma^{\prime(+)}\left(g^{\prime}\right)=\gamma^{\prime(+)}\left(g^{\prime}\right)=\frac{g^{\prime 2}}{8 \pi^{2}}\left(\begin{array}{cc}
-2 & 8 \\
0 & 7 / 3
\end{array}\right)+0\left(g^{\prime 4}\right)
\end{aligned}
$$

and

$$
\gamma^{\prime(-)}\left(g^{\prime \prime}\right)=\frac{g^{12}}{8 \pi^{2}}\left(\begin{array}{cc}
-8 & -B \\
0 & 7 / 3
\end{array}\right)+C\left(g^{\prime 4}\right)
$$

On removing the b-quark there are again two operators which enter. They have the same forn as $o_{7}^{( \pm \pm)}$and $o_{8}$ defined in Eqs. (3.20) and (3.21) except that $\mathrm{in} 0_{8}$ the factor of $\mathrm{m}_{\mathrm{c}}^{\prime 2} / \mathrm{g}^{\prime 2}$ is replaced by $\mathrm{m}_{\mathrm{c}}^{\prime \prime 2} / \mathrm{g}^{\prime \prime 2}$. The corresponding snomalous dimension matrices for these operators are

$$
\begin{aligned}
& \gamma^{\prime \prime(+)}\left(g^{\prime \prime}\right)=\frac{8^{\prime \prime 2}}{8 \pi^{2}}\left(\begin{array}{cc}
4 & -24 \\
0 & 5 / 3
\end{array}\right)+i\left(g^{\prime \prime 4}\right) \\
& \gamma^{\prime \prime}{ }^{(+-)}\left(g^{\prime \prime}\right)=\gamma^{\prime \prime}(+)\left(g^{\prime \prime}\right)=\frac{g^{\prime \prime 2}}{8 \pi^{2}}\left(\begin{array}{cc}
-2 & 8 \\
0 & 5 / 3
\end{array}\right)+c\left(g^{\prime \prime 4}\right) .
\end{aligned}
$$

and

$$
Y^{\prime \prime}(-)\left(g^{\prime \prime}\right)=\frac{g^{\prime \prime 2}}{8 \pi^{2}}\left(\begin{array}{cc}
-8 & -8 \\
0 & 5 / 3
\end{array}\right)+O\left(g^{\prime 4}\right)
$$

These are the same matrices as In Egs. (3.24) except that the 8-8 entry has changed froth $7 / 3$ to $5 / 3$ corresponding to the change of the number of flavors from 5 to $4 \mathrm{In}$ the coupling congtant renormalitation (1.e., a-function). Finaliy on reneving the chan quark only on operator proportional to $\mathrm{O}_{8}$ appears. Carrying through the steps of succergively traating the $t$. $b$ and $a$ quarks as heavy and removing them from explfcitly appraring in the theory using the $2 \times 2$ anumblous dimension matrices abcye vields the following analytic approximation for $\boldsymbol{*}_{3}$ : 


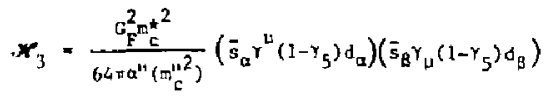

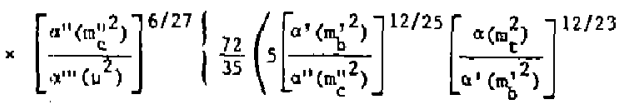

$$
\begin{aligned}
& \left.+2\left[\frac{a^{\prime}\left(m_{b}^{\prime 2}\right)}{a^{\prime \prime}\left(m_{c}^{\prime \prime 2}\right)}\right]^{5 / 25}\left[\frac{\alpha\left(m_{b}^{2}\right)}{a^{\prime}\left(m_{b}^{\prime 2}\right)}\right]^{12 / 23}-7\left[\frac{a^{\prime}\left(m_{b}^{\prime 2}\right)}{a^{\prime \prime}\left(m_{c}^{\prime 2}\right)}\right]^{5 / 25}\left[\frac{\alpha\left(m_{c}^{2}\right)}{\alpha^{\prime}\left(m_{b}^{\prime 2}\right)}\right]^{7 / 23}\right) \\
& \times\left[\frac{a\left(m_{t}^{2}\right)}{a\left(m_{t}^{2}\right)}\right]^{12 / 21}+\frac{4 a}{143}\left(13\left[\frac{\alpha^{\prime}\left(m_{b}^{2}\right)}{a^{\prime \prime}\left(m_{c}^{\prime n^{2}}\right)}\right]^{-6 / 25}\left[\frac{a\left(\sigma_{b}^{2}\right)}{a^{\prime}\left(m_{b}^{\prime 2}\right)}\right]^{-6 / 23}-2\left[\frac{a^{\prime}\left(m_{b}^{2}\right)}{a^{\prime \prime}\left(m_{c}^{\prime 2}\right)}\right]^{5 / 25}\right.
\end{aligned}
$$

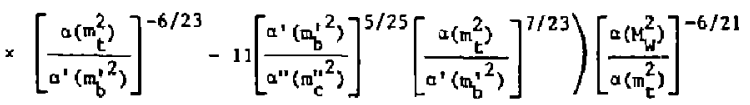

$$
\begin{aligned}
& +\frac{24}{899}\left(-31\left[\frac{a^{\prime}\left(m_{b}^{2}\right)}{a^{\prime \prime}\left(m_{c}^{\prime 2}\right)}\right]^{-24 / 25}\left[\frac{\alpha\left(m_{c}^{2}\right)}{a^{\prime}\left(m_{b}^{\prime 2}\right)}\right]^{-24 / 23}+2\left[\frac{a^{\prime}\left(m_{b}^{\prime 2}\right)}{a^{\prime \prime}\left(m_{c}^{\prime \prime 2}\right)}\right]^{5 / 25}\right. \\
& \left.\left.\times\left[\frac{a\left(m_{t}^{2}\right)}{a^{\prime}\left(m_{b}^{\prime 2}\right)}\right]^{-24 / 23}+29\left[\frac{a^{\prime}\left(m_{b}^{2}\right)}{a^{\prime \prime}\left(m_{c}^{\prime 2}\right)}\right]^{5 / 25}\left[\frac{a\left(m_{t}^{2}\right)}{a^{\prime}\left(\frac{\left.m_{b}^{2}\right)}{b^{2}}\right.}\right]^{7 / 23}\right)\left[\frac{a\left(m_{L}^{2}\right)}{a\left(m_{t}^{2}\right)}\right]^{-24 / 21}\right\} \text {. }
\end{aligned}
$$

The matrix elements of the three parts of the effective Hariltonfan for $k^{0}-\bar{k}^{\mathrm{U}}$ tolxing $1 .$. Eqs. $(3.15),(3.16)$ and $(3.26)$ are to be evaluated using the tuass independent $\overline{\text { MS }}$ subtraction scheme in an effective theary of strong interactions with three light quark flavors $u$, $d$ and $s$. The effects of QCD can be ascertained by comparing $\boldsymbol{x}_{1}, \mathscr{X}_{2}$ and, $\boldsymbol{x}_{3}$ given by Eqs. (3.15), (3.16) and (3.26) with theit f ree quatk values

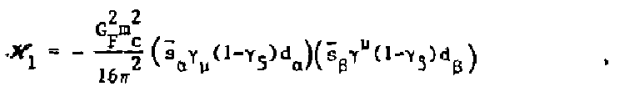

$$
\begin{aligned}
& x_{2}=-\frac{C_{F}^{2} m^{2}}{16 \pi} \pi^{2}\left(\bar{s}_{Q} \gamma_{\mu}\left(1-\gamma_{5}\right) d_{Q}\right)\left(\bar{s}_{B} \gamma^{H}\left(1-\gamma_{5}\right) d_{B}\right) \text {, }
\end{aligned}
$$

and

$$
\boldsymbol{x}_{3}=-\frac{G_{E}^{2} m_{c}^{2}}{16 \pi^{2}} \&\left(\frac{a_{t}^{2}}{a_{c}^{2}}\right)\left(\bar{s}_{a} \gamma_{\mu}\left(1-r_{g}\right) d_{\alpha}\right)\left(\bar{s}_{B} \gamma^{\mu}\left(1-\gamma_{5}\right) d_{\beta}\right) .
$$

These are derived by integrating the heavy $t$ and c-quark flelds out of the expressions given In Eq5. (3.3) and keeping only the leading contribution in the large quark masses $m_{c}$ and $m_{c}$.

\section{Numertcal ResuIts}

It is now possible to calculate (for given values of the patametera) the coefficient of $(\bar{s} d) \psi_{-A}(\bar{s} d) y-A$ in the pleces $\mathscr{X}_{1}, X_{2}$ a*d,$X_{3}$ of the effective $\Delta S=2$ Handitontan for $K^{\circ}-\bar{K}^{\circ}$ mixing and decemine the magntcude of the QCD ef fects by comparing these results with the1r free quark values. Unllke the case of the effective Hamiltonlan for $\Delta S=1$ veak nonleptonic decays, ve have simple analytic expressions for the pleces $\boldsymbol{x}_{1}, \boldsymbol{X}_{2}$ and $\boldsymbol{H}_{3}$ of the effective Hamiliontan. In order to derive an analytic expression for $\boldsymbol{x}_{3}$ nev approxidations beyond the lording log:arithmic approxtation were introduced. However these are not expected to significantly alter the numerical regults. (The skeptical reader can verify this by using the resules given in Appendix B to perform the calculation keepting all elght operatorf.) 
Again the outlook ts basically qualitative. The QCD effects have been computed in the leading logarithmic approximation and the c-quark mass was treated as a large quantity. For example, dispersfve pleces which ardec when the two u quarks in the loop of the box diagram show In FIg. 7 bind to form a low mase hadronic stace, have been beglected in comparison with picces that contain explicit factorg of the heavy c-quark mass. This is certainiy a crude approximation, but it has the advantage of being a systematic expansion and other contributions are not expected to be larger than the ones computed.

The effective Hamiltonlan for $k^{\circ}-\bar{K}^{\circ}$ mixing differs from that for the $\Delta S=1$ wesk nonleptonic decays in that at the final stage only one operator $(\bar{G} \mathrm{~d}){ }_{V-A}(\overline{B d}) V_{V-A}$ appears. Since any renormalization point Jependence in the wilson coefficients is cancelled by zenonalization point dependence of the matrix elements of this operator (at least if the H1lson caeffictents are computed exactly) the w1lson coeffictencs of this operator in $x_{1}, x_{2}$ and $x_{3}$ all have the same $\mu$ dependence. Unfortunately, the matrix clements cannot be calculated exactly so that some final prodictions may not appear renomalization point independent. However the quantity Im $\left\langle\mathbf{K}^{\circ}\left|\mathrm{H}_{\text {eff }}\right| \Delta S|=2| \overline{\mathrm{K}}^{\circ}\right\rangle / \mathrm{Re}\left\langle\mathrm{K}^{\circ}\left|\mathrm{H}_{\mathrm{eff}}^{|\Delta S|=2}\right| \overline{\mathrm{K}}^{\circ}\right\rangle$, which will be of interest, is Independent of the matrix elenents of (ds) $v-A$ (ds) $v-A$ and so predictions for it will also be free of renormblization point dependence.

To 1nvegtigate the effective Ham1ltontan for $\mathrm{K}^{\circ}-\overline{\mathrm{K}}^{\mathrm{O}}$ mixing values for the QCD running fine structure constant $a\left(Q^{2}\right)$, the values of the heavy massug and $\mu^{2}$, or alternatively $a^{\prime \prime \prime}\left(u^{2}\right)$, are required. For a $\left(u^{2}\right)$ ve again use

$$
a\left(Q^{2}\right)=\frac{12 \pi}{33-2 N_{f}} \frac{1}{\log \left(Q^{2} / A^{2}\right)}
$$

and take $h^{2}=0.1 \mathrm{GeV}^{2}$ and $\Lambda^{2}=0.61 \mathrm{GeV}^{2}$. The number of quark flavors 1.5 $\mathrm{H}_{\mathrm{f}}=6$ for the fine structure constant in the s1x-quark theory a(Q $\left.\mathrm{Q}^{2}\right)$, whlle $a^{\prime}\left(Q^{2}\right), a^{\prime \prime}\left(Q^{2}\right)$ and $a^{\prime \prime \prime}\left(Q^{2}\right)$ have $N_{F}=5,4$ and 3 respectively, as they perta1n to effective cheories of strong inceractions with those corresponding number of quark flavorg.

The quant1ty $m_{c}^{*}$, un $11 \mathrm{ke} \mathrm{m}_{\mathrm{c}}^{\prime \prime}$, $1 \mathrm{~s}$ free of renomalization point dupendence and hence more appropriately assoctated with the mass scale character 1zed by charmonium spectroscopy than til 1s. Thus for $\mathrm{t}_{c}^{*}$ the value $1.5 \mathrm{GeV}$ is taken on the basis of $\psi$ upectrogcopy. ${ }^{28}$ The difference between $m_{c}^{\prime \prime}$ and $\pi_{c}^{*} c$ can be neglected in the argument of the running fine ftructure constant in the leading logarithmic approximation but this is not the case for the explicit factor of the heavy f-quark mass squared which appears multiplying the operacor $(\overline{\mathrm{g} d}) \mathrm{v}_{\mathrm{A}}\left(\overline{\mathrm{s} d)} \mathrm{V-A}\right.$ in $\boldsymbol{x}_{1}$ and $\boldsymbol{x}_{3}$. similar remarks hold for the bottom and top quark masses. For mi the value $4.5 \mathrm{GeV}$ is taken on the basis of $T$ apectroscopy. ${ }^{28}$ Again for $\mathrm{m}_{t}^{*}$ values of $15 \mathrm{GeV}$ and $30 \mathrm{GeV}$ are used since the t-quark mass $1 \mathrm{~s}$ unknown at the present clase. $M_{W}=85 \mathrm{CeV}$. Stnce we shall be primarily concemed with the quanticy $I n\left\langle K^{\circ}\left|H_{\text {eff }}\right| \Delta S|=2| \bar{K}^{\circ}\right\rangle / \operatorname{Re}\left\langle K^{\circ}\left|H_{\text {eff }}\right| \Delta S|-2| \bar{K}^{\circ}\right\rangle$ which is Independent of $u$, only the value $a^{\prime \prime \prime}\left(u^{2}\right)=1$ is used. Values for the quantities $n_{1}$. $\eta_{2}$ and $\eta_{3}$, whlch are defined respectively as the ratios of the coefficlents of $(\overline{s d})_{v-A}(\overline{s d}) v-A$ in $\mathscr{X}_{1}, x_{2}$ and $x_{3}$ with strong interactions Included, to those in the free quark modol, are prestnted for the above 
choices of parametcrs in Table III. The free quark yalues of the coeff 1 cients ware deternined from Eqs. (3.27), (3.28) and (3.29) with $m_{c}=1.5 \mathrm{GeV}$, and $m_{t}-15 \mathrm{GaV}$ and $30 \mathrm{GeV}$. Note that the $Q \mathrm{CD}$ corrections tend to teduce the mangitude of $\mathscr{X}_{1}, \mathscr{X}_{2}$ and $, \mathscr{X}_{3}, \mathscr{X}_{1}$ being effected the least and $\mathscr{H}_{3}$ the most. The QCD correcticns to $\mathscr{X}_{2}$ and $\mathscr{H}_{3}$ are quite stable against variations of the parameters $m_{t}$ and $\Lambda$. However $\boldsymbol{x}_{1}$ changes by roughly a factor of 0.6 on golng from $\lambda^{2}=0.1 \mathrm{GeV}^{2}$ to $\Lambda^{2}=$ $0.01 \mathrm{GeV}^{2}$.

The results of this chapter can be camblned with chose of the previous one to make predictions for CP vlolation parameters in the katon system. This is done in the following chapter.
Table III

QCD correction factors $\eta_{1}, \eta_{2}$, and $\eta_{3}$ to the pieces, $x_{1}, x_{2}$, and $x_{3}$ of the effective Hamiltonian for $k^{\circ}-\bar{k}^{\circ}$ mixing.

\begin{tabular}{|c|c|c|c|}
\hline Parameters & $n_{1}$ & $n_{2}$ & $n_{3}$ \\
\hline $\begin{array}{l}\Lambda^{2}=0.1 \mathrm{GeV}^{2} \\
\mathrm{~m}_{\mathrm{t}}=15 \mathrm{GeV}\end{array}$ & 0.93 & 0.61 & 0.37 \\
\hline $\begin{array}{l}\Lambda^{2}=0.1 \mathrm{Gev}^{2} \\
m_{t}=30 \mathrm{GeV}\end{array}$ & 0.92 & 0.62 & 0.34 \\
\hline $\begin{array}{l}\Lambda^{2}=0.01 \mathrm{jeV} \mathrm{V}^{2} \\
m_{t}=15 \mathrm{GeV}\end{array}$ & 0.67 & 0.59 & 0.33 \\
\hline $\begin{array}{l}\Lambda^{2}=0.01 \mathrm{Gev}^{2} \\
\mathrm{~m}_{\mathrm{t}}=30 \mathrm{GeV}\end{array}$ & 0.67 & 0.60 & 0.33 \\
\hline
\end{tabular}




\section{CHAPTER IV}

CP VIOLATION PARATETERS OF THE $\mathbf{K}^{\circ}-\overrightarrow{\mathrm{K}}^{\circ}$ SYSTEM

The $K^{0}-\bar{K}^{\circ}$ system may be treated as a closed twa-gtate systea. Since the kaons decay, probabilfty is not conserved for this system and the time development 1 described by a $2 \times 2$ Hamiltontan matrix which is the sur of mass and widh matrices,

$$
H=M-\frac{\underline{L}}{2} \text {. }
$$

In the $\mathrm{K}^{\circ}-\overline{\mathrm{K}}^{\mathrm{O}}$ bastis $\mathrm{H}$ 1s given by

$$
H=\left[\begin{array}{ll}
H-1 \Gamma / 2 & H_{12}-1 \Gamma_{12} / 2 \\
M_{12}^{\star}-1 \Gamma_{12}^{\star} / 2 & M-1 \Gamma / 2
\end{array}\right]
$$

where

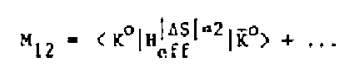

and

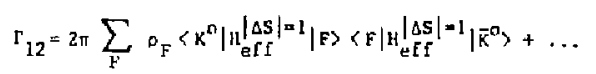

with $P_{F}$ the density of final states $F$. The effective Hanflconians

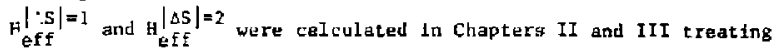
the strong interactions in the leading logarithmic approximation. The physical efgenstates for the system are

$$
\mathrm{K}_{\mathrm{S}}^{0}=\frac{1}{\left[2\left(1+|\varepsilon|^{2}\right)\right]^{1 / 2}}\left[(1+\varepsilon) \mathrm{K}^{0}+(1-\varepsilon) \overline{\mathrm{K}}^{0}\right]
$$

and

$$
K_{L}^{o}=\frac{1}{\left[2\left(1+|\varepsilon|^{2}\right)\right]^{1 / 2}}\left[(1+c) k^{o}-(1-\varepsilon) \bar{K}^{o}\right]
$$

wth mass and widch e1genvalues

$$
M_{S}-\frac{1 \Gamma_{S}}{2}=M-\frac{1 T}{2}+\left[\left(M_{12}^{*}-\frac{1 \Gamma_{12}^{*}}{2}\right)\left(M_{12}-\frac{1 \Gamma_{12}}{2}\right)\right]^{1 / 2}
$$

and

$$
M_{L}-\frac{1 \Gamma_{L}}{2}=H-\frac{1 \Gamma}{2}-\left[\left(M_{12}^{*}-\frac{\mathrm{IF}_{I 2}^{*}}{2}\right)\left(M_{12}-\frac{f \Gamma_{12}}{2}\right)\right]^{1 / 2} \text {. }
$$

The quanticy $E$ is given by

$$
\begin{aligned}
& \varepsilon=\frac{1 \mathrm{mH}_{22}+1 \mathrm{~m}_{12} / 2}{\left[\left(\mathrm{M}_{12}^{*}-\mathrm{i \Gamma _{12 } ^ { * }} / 2\right)\left(\mathrm{M}_{12}-1 \Gamma_{12} / 2\right)\right]^{1 / 2}+\mathrm{HeH}_{12}-1 \operatorname{Rer}_{12} / 2} \\
& =\frac{\left[\left(\mathrm{M}_{12}^{*}-1 \Gamma_{12}^{*} / 2\right)\left(M_{12}-1 \Gamma_{12} / 2\right)\right]^{1 / 2}-\operatorname{ReM}_{12}+\mathrm{iRe} \Gamma_{12} / 2}{-1]_{12}-\operatorname{Lm} \Gamma_{12}}
\end{aligned}
$$

Since $C P K^{0}=\bar{K}^{\circ}$ and $C P \bar{K}^{\circ}=K^{\circ}, I_{m T}$ and $I_{12 M}$ wre zeto when $C P$ is conserved and hence $\varepsilon$ is alsu zern. Note that the states $k_{S}^{0}$ and $k_{L}^{0}$ are noc in general orthogonal since probabllicy is not conserued in the kaon sygrem. In fact

$$
\left\langle K_{L} \mid K_{S}\right\rangle=\frac{2 R e \varepsilon}{\left(1+|c|^{2}\right)}
$$


so that $E$ is pure imaginary when the width matrix vantshes. I is not a physical quantity and its value depends on the phase convention one adopts far the kaon states or equivalently for the quark flelds. The standard phase convention is to have the $k^{\circ}+\pi(I=0)$ amplicude, $A_{0}$. teal. $A_{0}$ is defined by

$$
\left\langle\pi \pi(I=0)\left|H_{\text {eff }}^{|\Delta S|=1}\right| \mathrm{K}^{\circ}\right\rangle=\mathrm{A}_{0} \mathrm{e}^{1 \delta_{0}}
$$

where $\delta_{0}$ ts the $\pi \pi$ trakpin zero phase shift. If CP, or equivalently $T$, is conserved then ${ }_{C} C$ is automatically real since then

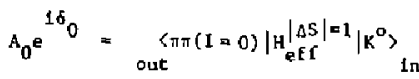

$$
\begin{aligned}
& =\operatorname{sut}_{\text {out }}^{\left\langle\pi(I=0)\left|\mathrm{I}^{-1} \mathrm{H}_{\mathrm{H}}\right| \Delta s\right\}=1} \mathrm{~T}\left|\mathrm{~K}^{0}\right\rangle \\
& =\left\langle\mathrm{K}_{\text {out }}\left|\mathrm{H}_{\text {eff }}\right| \Delta S|=1| \operatorname{tin}(1-0)\right\rangle
\end{aligned}
$$

The kion in state equals its out stace 35 (here in and out refer to strong inceractions) while for the two pion state below lnelastle threshoid 1sospln cunservation of the strong interactions implies that

$$
\begin{aligned}
|\pi \pi(I=0)\rangle & =|\pi n(1=0)\rangle \text { out out }\langle\pi \pi(I=0) \mid \pi \pi(1=0)\rangle \\
\text { in } & e^{21 \delta_{0}}|\pi \pi(I=0)\rangle \text { out. }
\end{aligned}
$$

Which when put into Eq. $(4.30)$ hives

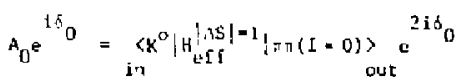

$$
\begin{aligned}
& =\left(A_{0} e^{1 \delta_{0}}\right)^{* 2 i \delta_{0}} \cdot A_{0} e^{i \xi_{0}} .
\end{aligned}
$$

In the six-quark Kobayashi-Waskawa model, where $C P$ is violated, unt can olways make A real by judlciously choosing how the phase \& enters the mitrix $y$ (which relotes mass and weak pigenstates). The chaice of $U$ we have made puts the phase only in the couplings of the heavy quarki (see Eq. (1.12) of Chapter I and Eq. (2.1) of Chapter II). Thus the CP violating couplings enter the ef fective Hatiltonian $\mathbb{H}_{\text {eff }}^{\Delta S=I}$ only ehrough Penguin-cype dhagrams whleh are pure $I=1 / 2$. Therefore the phose convention defined by the cnolet of quark fields in Eq. (1.12) of Chapter $I$ and Eq. (2.1) of Chaptar 11 corresponds to making the isospin two amplitude $A_{2}$, dertned by

$$
\left\langle\pi \pi(I=2)\left|H_{e f f}^{|\Delta S|-1}\right| K^{0}\right\rangle=A_{2} e^{10_{2}},
$$

real. $\delta_{2}$ is the $\pi \pi(I=2)$ phase shift. It will therefore be necessary to transform results calculated on the basis of this form of the weak couplings to that which corresponds to making ${ }_{0}$ real.

As was mentioned in the fincroduction, non-zero values for the physical quantities $n_{+}$and $n_{00}$ defined by 


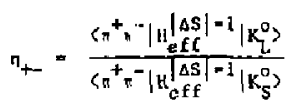

and

$$
\eta_{00}=\frac{\left\langle\pi^{0} \pi^{0}\left|H_{e f E}^{|\Delta S|=1}\right| K_{L}^{O}\right\rangle}{\left\langle\pi^{0} \pi^{0}\left|H_{e f f}^{\mid l S}\right|-1|| K_{S}^{O}\right\rangle}
$$

is a measure of $C P$ violation. The quantities $n_{+}$and $n_{00}$ can be expressed in terms of the 1sospin amplifudes $A_{0}$ and $A_{2}$ using the

followisg decompositions for s-wave twe-pion states:

$$
\begin{aligned}
& \left|\pi^{+} \pi^{-}\right\rangle=\frac{1}{\sqrt{3}}|\pi(I=2)\rangle+\sqrt{\frac{2}{3}}|\pi \pi(I=0)\rangle, \\
& \left|\pi^{0} \pi^{0}\right\rangle=\sqrt{\frac{2}{3}}|\pi \pi(I=2)\rangle-\frac{1}{\sqrt{3}}|\pi \pi(I=0)\rangle .
\end{aligned}
$$

Since the experimenral values of $n_{+-}$and $n_{00}$ are small (see

Eq. (1.10) of Chapter I) we dill drop terms 11 ke eImA and ELmA which are doubly CP violating. To lending order in CP violating quantities

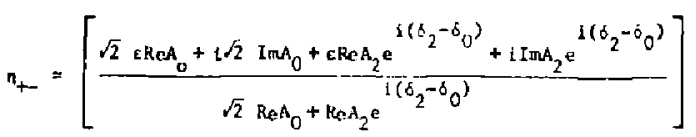

and

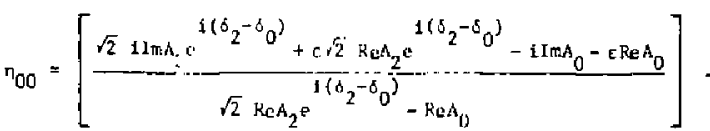

$$
\begin{aligned}
& \eta_{+}=\varepsilon+\varepsilon^{\prime} \\
& \eta_{00}=\varepsilon-2 \varepsilon^{\prime}
\end{aligned}
$$

when cerms of order $r$ : $\left(\right.$ ReA $\left.2 / A_{0}\right)$ are dropped (experimenrally $\left.\operatorname{ReA}_{2} / A_{0}=+1 / 20\right)$. Che ruantity $\varepsilon^{\prime \prime}$ is defined by

$$
c^{\prime}=\frac{i}{\sqrt{2}} e^{i\left(\delta_{2}-\delta_{0}\right)} \frac{I m A_{2}}{A_{0}},
$$

and the experimental values of $7_{+-}$and $\pi_{00}$ imply that

$$
\varepsilon^{\prime} / \varepsilon=-0.003 \pm 0.014
$$

To leading non-trivial order in CP violating quantities Eqs. (4,6) and $(4,7)$ become

$$
\begin{aligned}
& 4_{s}-\frac{1 \Gamma_{S}}{2}=4-\frac{1 \Gamma}{2}+\operatorname{Re} L_{12}-\frac{1 \operatorname{Re} \Gamma 17}{2} \\
& y_{2}-\frac{I \Gamma L}{2}=y-\frac{I T}{2}-\operatorname{Re} M_{12}+\frac{I R e \Gamma_{12}}{2}
\end{aligned}
$$

and

$$
c=\frac{1\left(\operatorname{Im}_{12}+i \operatorname{ImL}_{12}\right)}{\frac{1}{2}\left(\Gamma_{S}-\Gamma_{L}\right)+i\left(M_{S}-M_{L}\right)} \text {. }
$$

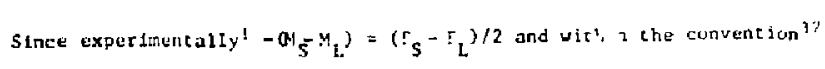
$A_{0}$ real $\left(\operatorname{Im} \Gamma_{12} / \operatorname{lm} \mathrm{m}_{12}\right) \leqslant 1 / 10$, it follows that when $A_{0}$ is chosen $\mathrm{rtal}$

$$
=\frac{1}{2 \sqrt{2}} e^{1-/ 4} \frac{1 \pi 412}{\mathrm{Re}^{4} \frac{12}{12}} .
$$




\section{Predictions for $\varepsilon^{\prime} /$}

In Chapter II it was noted that fif the motrix elements of the operator $Q_{6}$, in the effective Hamiltonian $6 . .2 \Delta S=1$ weak nonleptonic dacays, contribute significancly in $K+\pi$ decays then they wlll give the largest 1maginary $C$ violating parts to the $K \rightarrow \pi n$ amplitude. 16 Recall also that $Q_{6}$ arose from Penguin-type diagrams and has a $(V-A) O(V+A)$ chiral structure which may lead to enhanced matrix elements. Let $f$ be the fraction of che $K \rightarrow \pi n(1=0)$ amplitude due to matrix elements of $Q_{6}$ when th. $C P$ violating phase $\delta$ is set to zero. It is important to reallze that the value of $\mathrm{f}$ is strongly renomalizaL ton point dependent. In fact, In the leading logarithmic approxination, $f$ would be almost zero if the renotmalization point was equal to the charm quakk mass. This may seem gomewhat paradoxical since the Penguin-type diagrams are supposed to be the nource of the $\Delta I=1 / 2$ rule which ts a physical ef feet independente of $\mu$. However, one should keep in mind that a given dlagram in perturbation theory contributes, In ganeral, to the Hilson coefficlents and matrix clements of many of the operators in the effective Hamilconian. Consider for cxample the lowest order Penguin-type diagram with $u$ and $c$ quarks in the loop. This diagram not only gives a contribution to the Wilgon coefficient

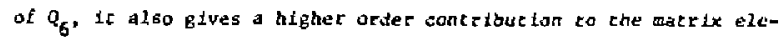
ments of $Q_{1}$ and $q_{2}$. How much goes into matrix elements of $q_{1}$ and $q_{2}$ depends (within the $\overline{M S}$ regularizat lon scheme) on the value of the renormalization polnt mass. In order to make predictions one chooses I to be at the typlcal Iight hadronic maso scale for the problam. It Ls then hoped thac enough of the features of the strong inceractions have been included in the wilson coefficients so that a simple estimate (for example using the naive quark model or bag model) of the matrix elenerts will lead to 3 gualitative underscanding of the problent. He shall agsume that there exiyts some " (near the typlcal 1 ight hadronlc mass scale) where the fraction $f$ is large. Then ar chis rcnormalization point the total amplitude for $K+\pi \pi(I=0), A_{0}$, is glven by

$$
A_{0}=A_{0}^{(\delta=0)}+1\left[A_{0}^{(\delta=0)} I m C_{6} / \operatorname{Re} C_{6}\right.
$$

where $A_{0}^{(\delta=0)}$ is the $K^{\circ} \rightarrow \pi j(I=0)$ amplifcude when the GP violation parameter $f$ is sec co zero. That is

$$
\left\langle 2 \pi(\mathrm{I}=0)\left|\mathrm{H}_{\mathrm{eff}}^{|\Delta S|=1}(\delta=0)\right| \mathrm{K}^{\circ}\right\rangle=A_{0}^{(\delta=0)} \mathrm{e}^{1 \delta_{0}} .
$$

As uas remarked previously, $A_{0}^{(\delta=0)}$ is reat. $C_{6}$ denotes the wilson coefficient of the operator $Q_{6}$ in the effective Hamiltonfan for $\Delta S=1$ wesk nonleptonic decays. $C_{6}$ was computed in Chapter III and values are presented in tables $I$ and II for various chotees of QCD parameters.

In addition there is CP violation in the kaon mass matrix. Frod Chapter III it follows that 


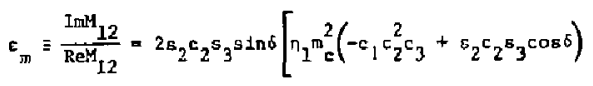

$$
\begin{aligned}
& +n_{2} m_{t}^{2}\left(c_{1} s_{2}^{2} c_{3}+s_{2} c_{2} s_{3} \cos \delta\right)+\pi_{3} m_{c}^{2} 2 n\left(m_{t}^{2} / m_{c}^{2}\right) \\
& \left.\times\left(c_{1} c_{2}^{2} c_{3}-c_{1} s_{2}^{2} c_{3}-2 s_{2} c_{2} s_{3} \cos \delta\right)\right]\left[n _ { 1 } m _ { c } ^ { 2 } c _ { 2 } ^ { 2 } \left\{\left(c_{1} c_{2} c_{3}-s_{2} b_{3} \cos \delta\right)^{2}\right.\right. \\
& \left.-\mathrm{s}_{2}^{2} \mathrm{~s}_{3}^{2} \sin ^{2} \delta\right\}+\pi_{2} \mathrm{~m}_{\mathrm{t}}^{2} \mathrm{~s}_{2}^{2}\left\{\left(\mathrm{c}_{1} \mathrm{~s}_{2} \mathrm{c}_{3}+\mathrm{c}_{2} \mathrm{~s}_{3} \cos \delta\right)^{2}-c_{2}^{2} \mathrm{~s}_{3}^{2} \sin ^{2} \delta\right\} \\
& +2 \pi_{3} m_{c}^{2} \ln \left(u_{z}^{2} / m_{c}^{2}\right) c_{2} s_{2}\left\{\left(c_{1} c_{2} c_{3}-s_{2} s_{3} \cos \delta\right)\left(c_{1} s_{2} c_{3}+c_{2} a_{3} \cos \delta\right)\right. \\
& \left.+\varepsilon_{2} s_{2} s_{3}^{2} \sin ^{2} \delta \phi\right]^{-1}
\end{aligned}
$$

Th1s expression is quice compltcacad; hewever, in the limit where $a_{1}$ and $s_{3}$ are treated as small quantictes it simpliffes to

$c_{m}=2 s_{2} c_{2} s_{3} s$ ind $\left\{\frac{-c_{2}^{2} m^{2} n_{1}+s_{2}^{2} m_{t}^{2} n_{2}+\left(c_{2}^{2}-s_{2}^{2}\right) m_{c}^{2} \ln \left(\frac{m^{2}}{\frac{t}{2}} m_{c} n_{3}\right.}{c_{2}^{4} m_{c}^{2} n_{1}+s_{2}^{4} m_{t}^{2} n_{2}+2 s_{2}^{2} c_{2}^{2} m_{c}^{2} 2 n\left(\frac{m^{2}}{m^{2}}\right) n_{3}}\right\} \cdot(4.26)$

$\eta_{1}$, $\eta_{2}$, and $n_{3}$ are the QCD corrections to the three portions of thr effective $\Delta S=2$ Hamlltonian $, \mathscr{H}_{1}, \mathscr{W}_{2}$, and $\mathscr{H}_{3}$ respectively. The quantitles $\eta_{1}, j \in\{1,2,3\}$, were computed in chapter 111 and values of $n_{1}, n_{2}$, and $n_{3}$ for typical QCD paraneters are presenced In Table III, Noto that all renomaltzat ton polnt dependence drops out of the expression for $\varepsilon_{\pi}$. If $f$ were zero then the ariplitude $s_{0}$ glven in Eq. $(4.23)$ would be ceal and It follows from Eq. (4.22) that $\varepsilon_{\mathrm{m}}$ would then be proportional to the CP violation parameter $\varepsilon$. However, we are faterested in the case where $f$ is a large fraction. Then

$$
A_{0}=A_{0}^{(\delta=0)} e^{1 E}
$$

where $\varepsilon=\mathrm{EIaC}_{6} / \mathrm{ReC}_{6}$. The standard phase convent $\mathrm{I}_{\text {, }} \mathrm{A}_{0}$ real, may be accomplished by readjust $\mathrm{Ing}$ the phase of the strange quark field

$$
s+e^{15} s
$$

so chat

$$
\begin{aligned}
& \left|\mathrm{k}^{0}\right\rangle+\mathrm{e}^{-1 \xi}\left|\mathrm{k}^{0}\right\rangle, \\
& \left|\overline{\mathrm{k}}^{0}\right\rangle+\mathrm{e}^{+1 \xi}\left|\overline{\mathrm{k}}^{0}\right\rangle .
\end{aligned}
$$

At the sane time

$$
\frac{\mathrm{ImM}_{12}}{\mathrm{ReM}_{12}}+\left(\varepsilon_{\mathrm{m}}+25\right),
$$

where $c_{n}$ is gatuen by Eq. (4.25). It Eollows from Eq. (4.22) chat the CP violation parameter : is given by

$$
E=\frac{1}{2 \sqrt{2}} e^{1 \pi / 4}\left(E_{\mathrm{q}}+2 \xi\right)
$$

Im and 25 give, in general, comparable contributions to $t$. The phase angle of $\pi / 4$ originates from the $K_{L}^{O}$ and $k_{S}^{o}$ mass and width values and has the precise value of $43.8^{2}=0.2^{*}$ just as in the superweak model. Ia general no prediction can be made for $c$ since the angles ${ }_{2}, 9_{3}$. and $\delta$ can be adjusced to fit the experimental value of $c$. The ochar 
CP violation parameter is, ' defined in Eq. (4,:b), (P vivlation from the Penguin-type operator $Q_{6}($ with $1=1 / 2)$ carnot enter the $A_{2}$ amplitude which involves a $\Delta I=3 / 2$ transition. Houever through the readjustment of the kaon phases to make $A_{0}$ real $A_{y}$ pifeks up on imakiagry part proportional to $t$ and

$$
\therefore=\frac{1}{20 \sqrt{2}} e^{1 \pi / 4}(-6)
$$

where the experimental value of the $n \pi$ phase shifts $\delta_{0}$ and $\delta_{2}$ together with ReA $A_{2} / A_{0}=+1 / 20$ have been used. The experdmentul value of the phase angle which we have approxfmated by $\pi / 4$ is $37^{\circ}: 6^{\circ}$. Conbining Eqs. $(4,31)$ and $(4,32)$ giver

$$
c^{\prime} / c=\frac{1}{20}\left(\frac{-25}{\varepsilon_{\pi}+2 \tau}\right) .
$$

In general $\varepsilon / c$ like $t_{\text {tin }}$ Lis a compllcated function of the cabibbo type angles $0_{1}, 0_{2}, \bar{g}_{3}$ and the phase $\varepsilon$. Examination of this function using results presented in Tables 1 . 11 , and III revials that values of $1 / 6$ ac the fraction of a percunt level are eypical. ${ }^{38}$ for example, when $s_{1}$ and $s_{3}$ are treated ns small quantitics boch 'n and $:$ are proporLlonal to $s_{2} \mathrm{c}_{2} s_{3}$ sins and all depondence on ${ }_{3}$, and 3 drops out of

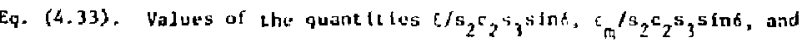
c $/$ e for this care are 1 lated in Tables IV and $v, t_{2}=15^{\circ}$ and $f=0.75$ were used for the tables. Inspection of the resules indicate chat values [roos $3 \times 10^{-3}$ to $3 \times 10^{-2}$ are typtaal cor $1 / 6$ when $s_{3}$ is a small quantity. Smallar values of $h$ or $f$ ran give smaller values for $c^{\prime} / c$. The quadrant if the phase is can be adjusted to fit the
Tatle IV

Values of the quant $15 y$, which leads to $\mathrm{CP}$ violation in decay amplitudes; $c_{m}$, the contribution

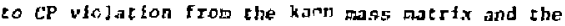
resuleting ratio of $\mathrm{CP}$ y lolation paramecers $\varepsilon^{1 / \varepsilon}$. These are ealculated with $s_{3}, s_{1}$ treated as small

\begin{tabular}{|c|c|c|c|}
\hline Parameters & $\therefore / \mathrm{fs}_{2} \mathrm{c}_{2} \mathrm{~s}_{3} \mathrm{sin}$ & $\varepsilon_{\mathrm{rt}} / s_{2} c_{2}{ }^{s} 3^{\sin \delta}$ & $c / \varepsilon$ \\
\hline$\alpha\left(\mu^{2}\right)=0.75, \pi_{t}+15 \operatorname{cec}$ & $-\left(0.42+s_{2}^{2}\right)^{-1}$ & 3.2 & $1 / 27$ \\
\hline$x\left(e^{2}\right)=1.00, \pi_{e}=15 \mathrm{GeV}$ & $-\left(0.65+s_{2}^{2}\right)^{-1}$ & 7.2 & $1 / 49$ \\
\hline$a\left(u^{2}\right)=1.25 . m_{t}=25 \mathrm{cov}$ & $-\left(0.81+s_{2}^{2}\right)^{-1}$ & 1.2 & $1 / 64$ \\
\hline$s\left(u^{2}\right)=0.75, m_{t}=30 \mathrm{cov}$ & $-\left(0.33+s_{2}^{2}\right)^{-1}$ & 16 & $1 / 65$ \\
\hline$a\left(\mu^{2}\right)=1.00, m_{t}=30 \mathrm{ceV}$ & $-\left(0.31+s_{2}^{2}\right)^{-1}$ & 16 & $1 / 103$ \\
\hline$a\left(v^{2}\right)=1.25, \mathrm{~m}_{t} \cdot 30 \mathrm{GeV}$ & $-\left(0.62+s_{?}^{2}\right)^{-1}$ & 16 & $1 /\{2\}$ \\
\hline
\end{tabular}
quantitics, $\theta_{2}=15^{\circ}, f=.75$, and $n^{2}=0.1 \mathrm{Gev}^{2}$. 
Table $V$

Same as Table IV but uith $A^{2}=0.01 \mathrm{GcV}^{2}$

\begin{tabular}{|c|c|c|c|}
\hline Parameters & $\zeta / \mathrm{Fs}_{2} \mathrm{c}_{2} \mathrm{~s}_{3} \mathrm{sin} \phi$ & $c_{m^{2} / t_{2} c_{2} s_{3} \ln \theta}$ & $c^{1 / c}$ \\
\hline$a\left(u^{2}\right)=0.75, m_{t}=15 \mathrm{GeV}$ & $-(1.46+$ & 8.9 & $1 / 161$ \\
\hline$a\left(\mu^{2}\right)=1.00, \pi_{t}=15 \mathrm{GeV}$ & .76 & 8.9 & $1 / 197$ \\
\hline $\mathrm{a}\left(\mathrm{p}^{2}\right)-1.25, \mathrm{~m}_{\mathrm{t}}-15 \mathrm{GeV}$ & $-\left(1.96+s_{2}^{2}\right)^{-1}$ & 8.9 & $1 / 220$ \\
\hline$a\left(\mu^{2}\right)=0.75, m_{t}=30 \mathrm{GeV}$ & $-(1.08+$ & 18 & $1 / 255$ \\
\hline$\alpha\left(u^{2}\right)=1.00, m_{t}=30 \mathrm{GeV}$ & $-\left(1.30+s_{2}^{2}\right)^{-1}$ & 18 & $1 / 308$ \\
\hline$a\left(\mu^{2}\right)=1.25, m_{t}=30 \mathrm{GeV}$ & $-\left(1.44+\mathrm{s}_{2}^{2}\right)^{-1}$ & 18 & $1 / 342$ \\
\hline
\end{tabular}

measured phase of $c_{\text {, then }} s_{1}$ and $s_{2}$ are treated as sasil quar ities, ve find that 6 should be in the upper half plane. ${ }^{16}$ Then - is nimust reat and posittive. For sone larger values of $s_{3}$ it la rossible to ith the measured phose of $E$ vith \& in the lower half $p l a n{ }^{39}$ and in this case $c / c$ hs almost real and negarive. The predictlons for $c^{\prime} / c$ presunted in Table [W are renormallzation point dependent. As was mentlaned before, our approach is to assume that a value of If exiats for whleh $f$ is large. Since we do not know exactly what w thls 15 , $c^{\prime} / c$ is calculated for several different choices of $a^{\prime \prime \prime}\left(\mu^{2}\right)$. Several authors have adopted a different approach. ${ }^{40}$ Since the real part of the Hilson coefficient $C_{6}$ depends on Integrat lons over virtual momenta primarily in the range $\nu^{2} \leqslant p^{2} \leqslant m_{e}^{2}$ whereas the imaginary part of $c_{b}$ depunds on integrations over virtual momenta primarily in the range $m_{c}^{2} \leq p^{2} \leq m_{t}^{2}$ a leading log calculal ton of the real part of the wilson cocficient for $Q_{b}$ is more uncertain than that of the imaginary part. Thus to calculate $\zeta$ they take the real part of the $K \rightarrow$ ता $(I=0)$ amplitude from the experimental uldth and rely on elther a vacuus insertion or bag model estlmate for the matrix elements of $Q_{6}$ to calculnte the imaginary part of $A_{0}$. This approach also involves an implicit cholce of $b$, namely that which makes the matrix element computation correct, and tends to give somewhat smaller values for the rat io $E \% / \mathrm{c}$

The presenc expertimental value is $e^{\prime} / \epsilon=-0.003: 0.014$ buc experlmenes are now planied ${ }^{4}$ which should be capable of measuring $r \%$ to the Eractina of a percent level. As such they mighe be capable of disxingulshing the six-qwark nodel, with importane contribuetons 
to $K+2 \pi$ decay from Penguin-type diegrame, f rom the superweak modelth where all CP violation origlnates from the kacn mass matrix and $\varepsilon^{\prime}=0$.
CHAPTER $v$

WEAK RADIATIVE HYPERON DECAYS ${ }^{4}$

The strangeness changing radiacive decays of hyperens have recelved congiderable attention by theorists.44,45 Many of the recent theoretical analyes have attempted to view these decays as arising f rom a local $s \rightarrow$ dr magneclc toment type crangition. ${ }^{45}$ Then the effect Ive Hagileonian for weak radtative decaya is

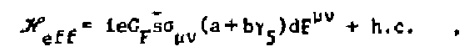

where $G_{F}$ is the Fermi constanc, e the electromagnetic charge of the elcction, $s$ and $d$ are strange and down quark fleids, and $F^{\text {tw }}$ is the electromagnatic fleld strength tensor.

The macrix elements of the effective Hamiltonian in Eq. (5.1) can be calculated reliably in the $\mathrm{SU}(6)$ quark model. ${ }^{16}$ The decays $\mathrm{B}_{1}+\mathrm{B}_{2} Y$ where $A_{1}$ and $B_{2}$ are baryons differing in strangeness by one unit are convenlently described in teros of helfity amplitudes ${ }^{47} g_{\lambda_{2}}, \lambda_{\gamma}$ labeled by the helicitles of the ourgoing baryon and photon. $g_{\lambda_{2}, \lambda_{Y}}$ 18 fust the Feynman plitude in the slluation where the initial baryon has spin component $\lambda_{1}=\lambda_{2}-\lambda_{y}$ alons the direction of the flasl baryon three-morrencun. When $B_{1}$ has spin compunent $\lambda_{1}$ along a giv $v_{t} n$ axis the resulting decay angular distribution is

$$
\frac{d \Gamma}{d \cos \theta}=\left.\frac{M_{2}|\vec{q}|}{4 \pi M_{1}} \sum_{\lambda_{2}, \lambda_{\gamma}}\left|g_{\lambda_{2}, \lambda_{Y}}\right|^{2} i_{\lambda_{1}, \lambda_{2}-\lambda_{\gamma}}^{J_{1}}(\theta)\right|^{2}
$$


so that

$$
\Gamma\left(g_{1}+B_{2} \gamma\right)=\frac{|\vec{a}| H_{2}}{2 \pi\left(2 J_{1}+1\right) H_{1}} \sum_{\lambda_{2}, \lambda_{\gamma}}\left|B_{\lambda_{2}, \lambda_{Y}}\right|^{2},
$$

where $\theta$ 1s the angle becween the given axis and the direction of the out-going baryon. The helicity amplitudes $8_{A_{2}}, \lambda_{Y}$ are easily calculated from the effectlve Hamilconian in Eq. (5.1) using SU(6) wave furctiong for the Infelal and final baryons. The helicity applitude contains several factors: first, a function which depends on the ovcrlap of the initial and final wave functions (when they are "separated" In momencum space by the photons momentum $\vec{q}) \mathbf{F}(\vec{q})$; second, a spif dependent factor $c_{\lambda_{2}, \lambda}$ whteh 15 essentially a Clebsch-Cordon coefficient aristag fram the spin part of the baryon wave fusctions; and third, a factor linear in the constants $a$ and $b$ of Eq. (5.1) This last factor is proportional co $G_{F} e(a-b) / a \mid$ when $\lambda_{Y}=+1(1 n$ whlch case the intetal s quark spin is parallel to the photon chrce momenrum) and proportional to $c_{F} e(a+b)|\vec{a}|$ when $x_{\gamma}=-1$ (1n whteh case the 1nitial quark spta is anciparallel to the phoran three momentu凶). Therefore

$$
\begin{aligned}
& 8_{\lambda_{2},+1}=2 \sqrt{2} G_{F} e|\vec{q}|(a-b) F(\vec{q}) C_{A_{2},+1} \\
& B_{\lambda_{2},-1}=2 \sqrt{2} G_{F} e|\vec{q}|(a+b) F(\vec{q}) C_{\lambda_{2},-1}
\end{aligned}
$$

The apin dependent factor from the quark model wave functions of the baryons is the same when all helicitles are reversed in stga, i.e.
$c_{\lambda_{2},+1}=C_{-\lambda_{2},-1}$. The overlap function $F(\vec{q})$ is normalized so in the nonrelativistic quark model $F(0)=1$. Inserting Eqs. (5.4) into

Eq. (5.3) gives

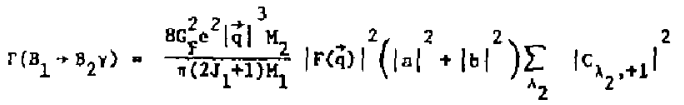

The only observed cadlative hyperon decay is $\Sigma^{+}+p \gamma$ with a branching rar10 of $(1.24 \pm 0.18) \times 10^{-3}$. For the orher weak radiative hyperon decays only upper llaits exist at the presenc time. NormalizIng the observed $\Sigma^{+} \rightarrow$ pr width, predictions for the other baryon decays can be made provided $\vec{f}(\vec{q})$ is slowly varying with $\vec{q}$. In thls cage the factor $\left(|a|^{2}+|b|^{2}\right)|F(\vec{a})|^{2}$ is determined from the observed $x^{+}+\mathrm{PY}$ width and the branching ratios for the other hyperon decays follow from this and the values of $\left.|\vec{q}|^{3} M_{2} /\left[(2]_{1}+1\right) M_{1}\right]$ and $C_{\lambda_{2}, \lambda_{y}}$. Predlcthons for the wak radrative hyperon decays are presented in Trable VI. There is a large disagreement betueen the predtcted rates for $\bar{\Xi}^{-} \cdot \bar{\Sigma}^{-} \gamma$ and $\Omega^{-}+\Xi^{-} \gamma$ and the experimental upper limits on these decay modes. 48 Thus it appears that the weak radiative decays of all the hyperons cannot arise fram a hocal menetic modent type transition.

So fat no dynamical assumptions an the origin of thi: effective Hatolitonlan in En. (5.1) have been made. In the 4-quark HelnbergSnlear model the coefficlents $a$ and $b$ can be calculated since diagrams like Fig. 8, with gluon corrections, are short distance doringled to the extent that the $y$-boson and the charm quark are very massive. 
Table vi

Predictions for weak radiasive decays of hyperons

based on the local magnetic moment transition.

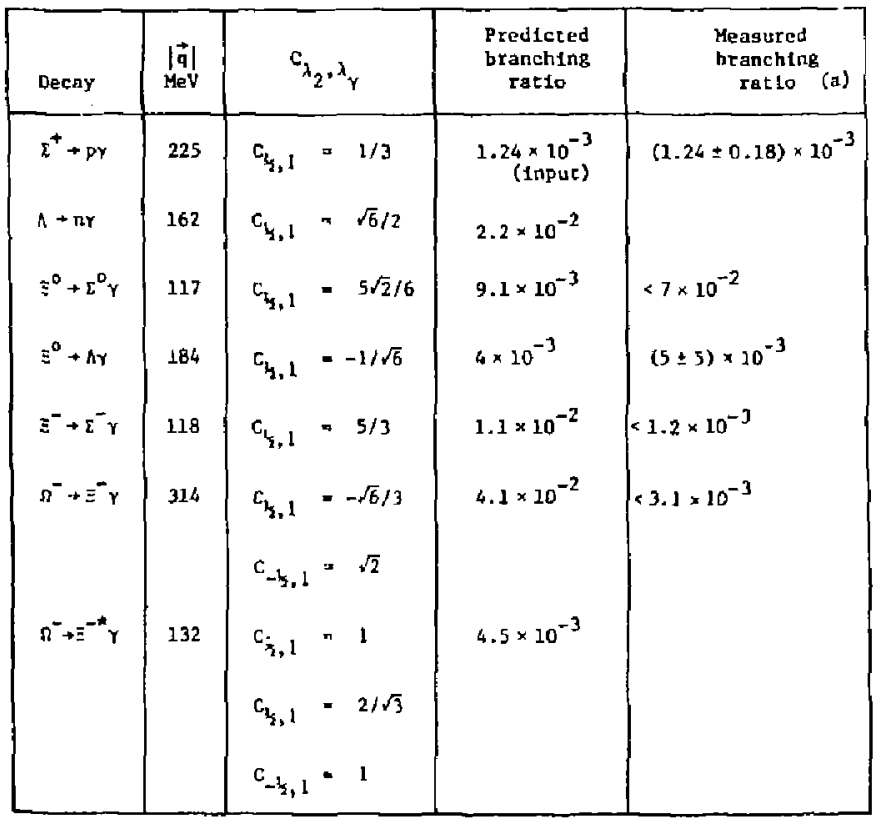
(a) The branching ratio for $\Xi^{0}+\Lambda^{0} \gamma$ to given as $(2.3 \pm 0.7) \times 10^{-3}$ in

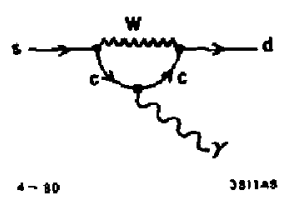

Fig. 8. Diagram which gives tige to a locis magnetic mosent transition in the absence of scrong interactions. 
Cocffictents a and butch are not suppressed by a factor of $\left(\frac{m}{b i}\right)^{2}$ first arlse from diagrams at the two loop level and hence the values of $a$ and $b$ are quite grall. These coefficients have been calculated In the standard 4-quark model by Shlfman, Vainshtein and Zakhavov. 49 They found

$$
\begin{aligned}
& a=\frac{\sin \theta c \cos \theta c}{\sqrt{2} 16 \pi^{2}} a\left(m_{s}+m_{d}\right) \\
& b=\frac{\sin r c_{c o s}^{\cos } c}{\sqrt{2} 16 \pi^{2}} \times\left(m_{d}-m_{s}\right)
\end{aligned}
$$

where

$$
\begin{aligned}
e & =-\left[\frac{a\left(m^{2}\right)}{a^{\prime}\left(u^{2}\right)}\right]^{16 / 27}\left\{2 / 7\left(\left[\frac{a\left(m_{c}^{2}\right)}{\alpha^{\prime}\left(u^{2}\right)}\right]^{-28 / 27}-1\right)\left[\frac{a\left(m_{w}^{2}\right)}{a\left(m_{c}^{2}\right)}\right]^{-12 / 25}\right. \\
& \left.+\frac{4}{5}\left(\left[\frac{a\left(m_{c}^{2}\right)}{a^{2}\left(\mu^{2}\right)}\right]^{-10 / 27}-1\right)\left[\frac{a\left(m_{w}^{2}\right)}{\alpha\left(m_{c}^{2}\right)}\right]^{6 / 25}\right\} .
\end{aligned}
$$

Pueting this into Eq. $(5,5)$ yields predictions for branching ratios of weak radtative hypezon decays of order $10^{-5}$ (or less).

If the locul megnetic moment transition given by the effective Hardltonian in 8 q. (5.1) Is not the mechanism for weak radiatlve decnys then what is? Oeher possible contributions come from the matrix elements of the effectve Handitontan for dS = I weak nonlegtonic decays evaluaced to order $*$ in the clectromagnelie interactions. Withyn the context of the pole modst, where the reak radiative decays are viewed as a weak nonleptonic transition followed bv the radiation of a photon or vice verss, the rates for weak radiative decays are related to those of the nonleptonlc decays. It is noceworthy that when the lockl magnetic moment transition is neglected the weak radiative decays of negatively charged baryons can only proceed through Pengutn-type diagrame (w1th a photon radiated off one of the quark legs in the Pengutn diagram or off a "spectator" quark leg). A meagurenent of branching ratlos for the decays $\bar{\Sigma}^{-}+E Y$ and $\Omega^{-}+\Sigma^{-} Y$ of order $10^{-3}$ sould be strong eutdence that the Penguin-type diagrams are Imporcant in the weak radiaclve dccays of hyperons and gualitative evidence that Penguin-type diagrans, which have been proposed as an explanation of the. $\Delta I=1 / 2$ rule, are important in the weak nonleptonic decays of hyperons.

The spectal role of Penguin-type diagrams (with a photon radiaced off one of the quark legs in the Penguin diagras or off a "spercator" quark leg) in the weak radiative decays of negatively charged hyperons leads one to belleve that a simflar effect should exist for the nonleptonic hyperon decays. Thls is examined in the next section.

1. The f/d Ratio in Nonlegtonke deak Hyperon Dersys Let $\mathrm{H}_{\mathrm{eff}}^{\mathrm{AC}} \mathrm{l,8}$ be the portion of the effective Haraltionian for weak nonleptonic decays that transforms like the sixth component of an octec under su(j) flavor. Let $B_{1}, 1 \in[1, \ldots 8\}$ denote the $\operatorname{spin} 1 / 2$ positive paryty baryon states in the octet. The s-wave nonleptanic hyperon decay amplitudes are related through PCAC in to the macrix eluments of the parity conserving parc of the effective nonleptonic Hamiltontan between baryon states differing in strangeness by one unit. 
The p-wave nonleptonic batyon decay amplitudes are not directly related through PCAC to matrix elements of the parity violating part of the effective nonleptonic Hamilconian due to the presence of pale terms. Hence we shall focus our attention on the s-wave anplitudes.

Define

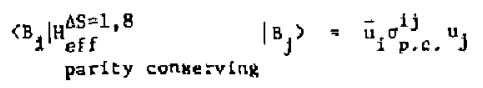

where $u_{f}$ and $u_{j}$ are Dirac spinors for the spin $1 / 2$ baryon states $B_{i}$ and $B_{f}$ (see Appendix $C$ ). Assuming that su(3) is a good symenetry the above matrix elements can be characterized by cwo reduced matrix cllmentas $f$ and $d$ and clebsch-Gordon coeffictents $E_{1 j k}$ and $d_{1 j k}$ in the following manner

$$
a_{p \cdot c .}^{i d}=-1 f_{b 1 j^{f}}+d_{f 1 j} d \text {. }
$$

where $r_{1 j k}$ and $d_{1 j k}$ are defined by the commutators and anticomatators of the SU(3) Gell Mann thatrices $\lambda_{1}$ (normallzed by $\operatorname{Tr}\left(\lambda_{i} \lambda_{f}\right)-2 \delta_{i f}$ ):

$$
\left[\lambda_{i}, \lambda_{j}\right]=21 \mathrm{f}_{1 j k} \lambda_{k}
$$

and

$$
\left\{A_{f}, \lambda_{j}\right\}=2 d_{i j k} A_{k}+4 / 3 s_{i j}
$$

Since the negatively charged hyperon transition matrix element ean only proceed through Penguin-type dlagrams (W exchange camnot occur between a11 $Q=-1 / 3$ quarks) and the contribution of the part of the
Hamileonian that is not octet (i.e, the 27) is known from expertment

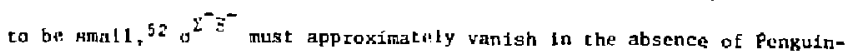
type dfagrams. Using the results in appendix $\mathrm{C}$ and the values of the coeffictents $f_{i j k}$ and $d_{1 j k}$. Biven in Table vil,

$$
a^{[-T-=}=\frac{1}{2}[f+d] .
$$

Thus ve conclude chat, in the absence of Penguin-type contributions to the baryon-baryon cransition matrix element $f / d=-1$. Th1s is not to say that if the coefficients of the opcrators $q_{7}, \ldots, Q_{b}$ in the effective Haniltonian for $\Delta S=1$ weak nonleptonic decays were set to zero then $f / d$ is necessarily equal to winus one. As was mentioned prev1ougly the Pengulnotype diagrams also give higher order contributions to the matrix elcments of $Q_{1}$ and $Q_{2}$. However, it is usually assuned that these are strall when $\mu$ is chosen to be at the eypical light hadronic mass scale which characterize the decay. Experimentelly a good fic to s-wave hyperon decay amplitudes occurs for $f=-2 d$. This is evidence that Penguln-type diagrams do play a significant role in weak nonleptonic hyperon decays. 
Table VII

Non zero $f_{i j k}$ and $d_{i j k}$. Non zero elements not Iisted in the table below can be derived by noting that $f_{i j k}$ is completely ant fsymetric and $d_{1 j k}$ ts completely symetric

\begin{tabular}{|c|c|c|c|}
\hline 1$\} \mathrm{k}$ & $E_{1\} k}$ & $1 \jmath k$ & $d_{i j k}$ \\
\hline 123 & 1 & 118 & $1 / \sqrt{3}$ \\
\hline 147 & $1 / 2$ & 146 & $1 / 2$ \\
\hline 156 & $-1 / 2$ & 157 & $1 / 2$ \\
\hline 246 & $1 / 2$ & 228 & $1 / \sqrt{3}$ \\
\hline 257 & $1 / 2$ & 247 & $-1 / 2$ \\
\hline 345 & $1 / 2$ & 256 & $1 / 2$ \\
\hline 367 & $-1 / 2$ & 338 & $1 / \sqrt{3}$ \\
\hline 458 & $\sqrt{3} / 2$ & 344 & $1 / 2$ \\
\hline \multirow[t]{7}{*}{678} & $\sqrt{3} / 2$ & 366 & $-1 / 2$ \\
\hline & & 377 & $-1 / 2$ \\
\hline & & 448 & $-1 / 2 \sqrt{3}$ \\
\hline & & 558 & $-1 / 2 \sqrt{3}$ \\
\hline & & 668 & $-1 / 2 \sqrt{3}$ \\
\hline & & 778 & $-1 / 2 \sqrt{3}$ \\
\hline & & 888 & $-1 / \sqrt{3}$ \\
\hline
\end{tabular}

CHAPTER VI

CONCLUDING REMARKS

In this report some of the 1 mplications of a posstble mechanism for the $A I=1 / 2$ rule, based on a prominent role for Penguin-type diagrams, were discussed. It was found that within the six-quark model for $C P$ violation an important role for Penguin-type dagrans in $K+\pi$ decays leads to values of $\varepsilon^{\prime} / \varepsilon$ of order a fraction of a percent. Thus the Kobayash1-Maskawa six-quark model for CP violation may be distinguishable from the superueak model where $\varepsilon^{\prime}=0$. In addition to gaining Insight into the relationshlp between $\mathrm{CP}$ violation and the $\Delta \mathrm{I}=1 / 2$ rule 1t was found that weak radiative hyperon decays may play a role in ascertaining the significance of Pengui,-type diagrams in the weak nonleptonic decays of hyperons.

In order to calculate $E^{1 / 6}$ the effective Hamilcontans for is $=1$ weak nonleptonic decays and $\Delta S=2 k^{\circ}-\overline{\mathbf{k}}^{0}$ mixlng were derlved by successfvely treating the $W$-boson, $t$-quark, b-quark, and $c$-quark as heavy and removing their flelds from explicitly appearing the theory.

Strong interaction effects were taken into account in the leading logarithraic approximation. It is hoped that the mechod for performing such calculations may prove usetul to workers in other areas (e.g., deep Inelastic scattering 3,53 and grand unified theorfes ${ }^{54}$ ) where the effective field theory formalism can be applied.

Most of the results derived in this report, while quantitative in principle, have been qualitative in thetr applctation to strange particle decays. This is partly because the trectment of the charm quark 
mass as large and using te as an expangion parameter ls suspect. 55 However, the greatest linftation an aur abllity to make quantitative predictions comes from diffleulties 10 ralculacing the matrix elements of the operators which enter the effectlve HamLltorian for $\Delta S=1$ veak nonleptonlc decays. These are renornalized local four-quark operators and any gerlous attempt to calculate their matrix elenents must deal with the dependence of these macrix elements on the renormalization point mass. In the vacuum insertion approximation, which is commonly used to evaluate these matrix elements, a renotmaltzed local faurquark operator is split into a product of renomalized quark bilinears. This completely destroys the renomelization point dependence of the macrix elements (note that $\left\langle\left|\left(\psi_{1} \psi_{2} \psi_{3} \psi_{4}\right)^{R}\right|\right\rangle \neq \sum_{n}\left\langle\left|\left(\psi_{1} \psi_{2}\right)^{R}\right| n\right\rangle x$ [n] $\left.\left(\psi_{3} \psi_{4}\right)^{\mathrm{R}} \mid \gg\right)$, and therefore if this appraximation is ever valid it can only be at one particular value of the renormalization polnt mass. This value is usually taker to be the cypical light hadronic mass scale which charicterizes the decay. Simflar remarks hold for bagmodel estimates of the matrix telements. ${ }^{56}$ Much further work is needed on this problem before it can be claimed that we have a quantitative understanding of the weak nonleptonle decays of kaons and hyperons.
APPENDIX A

In this appendix we outline the derivation of the equations and Rive numerical results for the quantities which appear in section 1 of Chapter II. In Suction I of Chapter II a rather fundamental role was played by the renormalization group Ecs. $(2.22),(2.32),(2.37)$ and $(2.48)$. To get Eq. $(2.22)$, for example, one merely applies $\mu \frac{d}{d \mu}$ to both aldes of Eq- (2.18) using

$$
\begin{aligned}
& \left.\left.\mu \frac{d}{d \mu}\left\langle\gamma 0_{t}^{( \pm)} \mid\right\rangle=\left(w \frac{\partial}{\partial \mu}+g(g) \frac{\partial}{\partial g}+\sum_{q} \gamma_{q}(g) m_{q} \frac{\partial}{\partial m_{q}}\right)\langle| o_{t}^{( \pm)}\right\}\right\rangle \\
& -\gamma^{( \pm)}(B)\left\langle\left|0_{t}^{( \pm)}\right|\right\rangle \text {, } \\
& \mu \frac{d}{d \mu}\left\langle\left|0_{1}\right|\right\rangle^{\prime}=\left(\mu \frac{\partial}{\partial \mu}+\beta^{\prime}\left(g^{\prime}\right) \frac{\partial}{\partial g^{\prime}}+\sum_{q} Y_{q}^{\prime}\left(g^{\prime}\right) m_{q}^{\prime} \frac{\partial}{\partial m_{q}^{\prime}}\right)\left\langle\left|o_{1}\right|\right\rangle+ \\
& --\sum_{j} r_{1 j}^{\prime}\left(8^{\circ}\right)\left\langle\left|0_{j}\right|\right\rangle^{\prime},
\end{aligned}
$$

and

$\mu \frac{d}{d \mu} B_{j}^{( \pm)}\left(\frac{m_{t}}{\mu}, g\right)=\left(\mu \frac{\partial}{\partial \mu}+B(g) \frac{\partial}{\partial g}+\gamma_{L}(g) m_{t} \frac{\partial}{\partial \Phi_{t}}\right) B_{j}^{( \pm)}\left(\frac{\omega_{t}}{\mu}, g\right)$.

In Eqs. ( $A, 1)$ and (A.3) the partial derivative with respect to $\omega$ is at constant $g$ and $m_{q}$, where $q \in\{u, d, \ldots, t\}$, while in Eq. (A.2) it is at constant $g^{\prime}$ and $m_{q}^{\prime}$, where $q \in[0, d, \ldots, b\}$.

The $\gamma^{( \pm)}(g)$ and the matrix $\gamma_{k j}^{\prime}\left(g^{\prime}\right)$ arise because the operators $0_{t}^{( \pm)}$ and $o_{1}$ are local four-fermion operators and require renormalization. The renormulization of the operators $o_{q}^{( \pm)}$at the one-loop level was 
considered in Refs. 5 and 6 where it was ghown that the $\gamma^{(t)}(\mathrm{g})$ are given by Eq. (2.12). From Eq. $(2,16)$, with $N_{f}=6$, it follows that

$$
a^{(+)}=\frac{6}{21} \quad, \quad a^{(-)}=-\frac{12}{21} \quad \text {. }
$$

At the one-loop devel the operators of undergo renomaltzmtion

$$
o_{j}^{a}=\sum_{k} z_{j k} o_{k}
$$

where a superscript " $a$ " denotes a bsre unrenormallzed quanticy. $z_{j k}$ is the matrix renormalization which axioes because of the composice nature of the local four-fertion operators $o_{j}$ - The renormalized operators are defined so that their matrix elements arc finite. The matrix $y_{1 j}^{\prime}\left(g^{\prime}\right)$ is defined by

$$
Y_{i j}^{\prime}\left(B^{\prime}\right)=\sum_{k} z_{1 k}^{-1} \mu \frac{d}{d \mu} z_{k j}
$$

Note that the $z_{j k}$ are a function of the coupling $g^{*}$ since the renoramlization of the operators of ts calculated in the effectiva 5 quark theory with that coupling. A atraightforward calculation of the "Infinite part" of the one-particle-irreduclble diagramg in Flg. 9, using Landau gauge, gives

$$
r_{1 j}^{\prime}\left(\mathrm{B}^{\prime}\right)=\frac{\mathrm{B}^{12}}{8 \pi^{2}}\left(\begin{array}{cccccc}
-1 & 3 & 0 & 0 & 0 & 0 \\
3 & -1 & -1 / 9 & 1 / 3 & -1 / 9 & 1 / 3 \\
0 & 0 & -11 / 9 & 11 / 3 & -2 / 9 & 2 / 3 \\
0 & 0 & 22 / 9 & 2 / 3 & -5 / 9 & 5 / 3 \\
0 & 0 & 0 & 0 & 1 & -3 \\
0 & 0 & -5 / 9 & 5 / 3 & -5 / 9 & -19 / 3
\end{array}\right)+O\left(\mathbf{g}^{14}\right) .
$$
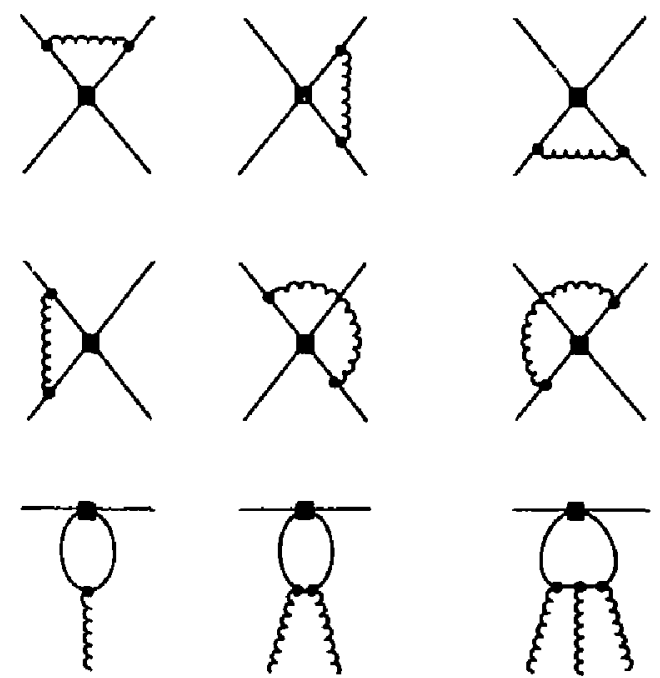

s.

387943

F1g- 9. Diagrams entering the calculation of the renotmalization of the local four-fermion operators (tepresented by the black box) through QCD effects. 
$-99-$

In the calculation of the renormalization of the local four-fermion operators, $a_{j}$, the masses of the light up, down, and strange quarks was set to zero. If this was not done the operators of would close under renomalization at the one-loop level but at the two loop level a transitian color magnetic moment term wust be added. However, the presence of such an operator docs not alter the wilson coeffictents of the lacal four-fermion operators. $o_{j}$, from their value calculated whth the light quark masses set to zero. The transition color magnet1c moment operator itself is explicttly proportional to a light quark mass yleiding Emall matrix elements. Also the Wilson coeffictent of -re mas.atic moment operator 18 expected to be small. These facts jusilfy our approximation of serting the $u, d$, and $s$ quark masse $\$$ to zero.

The matrix $\gamma_{1 j}^{\prime T}\left(8^{\prime}\right)$ can be diagonalized by the trangformation

$$
\sum_{k=2} v_{1 \ell}^{-1} \gamma_{2 k}^{\prime T}\left(g^{\prime}\right) v_{k J}=\delta_{1 j} r_{j}^{\prime}\left(B^{\prime}\right)
$$

where

$$
v_{h J}=\left(\begin{array}{lccccc}
0 & -.69483 & 0 & 0 & .70576 & 0 \\
0 & .69483 & 0 & 0 & .70576 & 0 \\
.15042 & .23161 & -1.253 & .16684 & -.10082 & .42681 \\
-.2089 & -.23161 & 1.0843 & .081196 & -.10082 & .02414 \\
.032942 & 0 & .10426 & .93924 & 0 & -.3322 \\
.61688 & 0 & .21323 & -.34513 & 0 & .28045
\end{array}\right)
$$

and
$-100-$

$$
r_{1}^{\prime}\left(8^{\prime}\right)=\frac{g^{\prime 2}}{B^{2}}\left(\begin{array}{l}
-6.8956 \\
-4 \\
-3.2429 \\
1.1166 \\
2 \\
3.1327
\end{array}\right)+6\left(g^{4}\right)
$$

Combiafng (A.10) vich the perturbative expanstion of $\mathrm{g}^{\prime}\left(\mathrm{g}^{\prime}\right)$ in Eq. (27) ylelds the $a_{j}$ of Eq. (28):

$$
. j=\left(\begin{array}{c}
-.8994 \\
-12 / 23 \\
-.42299 \\
.14564 \\
6 / 23 \\
.40861
\end{array}\right)
$$

Note that $a_{2}^{\prime}=a^{\prime(-)}$ and $a_{4}^{\prime}=a^{(+)}$where

$$
-\frac{y^{( \pm)}(x)}{B^{\prime}(x)}=\frac{2 a^{\prime}( \pm)}{x}+\operatorname{ceros} \text { findte at } x=0
$$

and

$$
\begin{aligned}
& r^{\prime(+)}\left(g^{\prime}\right)=\frac{g^{2}}{4 \pi^{2}}+\sigma\left(g^{\prime 4}\right) \\
& r^{\prime(-)}\left(g^{\prime}\right)=-\frac{g^{2}}{2 g^{2}}+O\left(g^{\prime 4}\right)
\end{aligned}
$$

The case where the botcom quark is treaced as very heavy is binilar to the above and ve simply state results: 


$$
r_{\operatorname{m}}^{\prime \prime}\left(g^{\prime \prime}\right)=\frac{8^{\prime \prime 2}}{8_{\pi}^{2}}\left(\begin{array}{cccccc}
-1 & 3 & 0 & 0 & 0 & 0 \\
3 & -1 & -1 / 9 & 1 / 3 & -1 / 9 & 1 / 3 \\
0 & 0 & -11 / 9 & 11 / 3 & -2 / 9 & 2 / 3 \\
0 & 0 & 23 / 9 & 1 / 3 & -4 / 9 & 4 / 3 \\
0 & 0 & 0 & 0 & 1 & -3 \\
0 & 0 & -4 / 9 & 4 / 3 & -4 / 9 & -20 / 3
\end{array}\right)+O\left(g^{\prime 1^{4}}\right)
$$

$Y_{\text {min }}^{\prime T}\left(g^{\prime \prime)}\right.$ is diagonalized by the transformation

$$
\sum_{2, h} w_{n Q}^{-1} \gamma_{2 k}^{\prime \prime T}\left(g^{\prime \prime}\right) w_{k m}=\delta_{n m} \gamma_{n}^{\prime \prime}\left(g^{\prime \prime}\right)
$$

where

$$
\mathrm{W}_{\mathrm{kO}}=\left(\begin{array}{llllll}
0 & .67552 & 0 & 0 & .70598 & 0 \\
0 & -.67552 & 0 & 0 & .70598 & 0 \\
-.13011 & -.33776 & -1.2092 & .14075 & -.11766 & .47246 \\
.18274 & .33776 & 1.1043 & .067129 & -.11766 & .80199 \\
-.02959 & 0 & .064119 & .96326 & 0 & -.30023 \\
-.65316 & 0 & .14969 & -.34859 & 0 & .23908
\end{array}\right) \text {, }
$$

and

$$
Y_{\mathrm{n}}^{\prime \prime}\left(\mathrm{g}^{\prime \prime)}\right)=\frac{\mathrm{g}^{\mathrm{n}}}{8 \pi^{2}}\left(\begin{array}{l}
-7.0428 \\
-4 \\
-3.501 \\
1.0974 \\
2 \\
2.8909
\end{array}\right)+C\left(\mathrm{~g}^{\prime \prime 4}\right)
$$

It rullows from (, 17$)$ and the perturbacive expansion of $B "(g ")$ that

$$
a_{\text {a }}=\left(\begin{array}{c}
-.84514 \\
-12 / 25 \\
-.42012 \\
.13169 \\
6 / 25 \\
.34691
\end{array}\right)
$$

Again $a_{2}^{\prime \prime}=a^{\prime \prime(-)}$ and $a_{4}^{\prime \prime}=a^{\prime \prime(+)}$.

When the heavy chars quark expansion is performed only the Elve operatora $Q_{1}, Q_{2}, Q_{3}, Q_{5}$, and $Q_{6}$ def ined in $E q .(-4)$ are required. He find that

$$
r_{p r}^{\prime \prime \prime}\left(g^{\prime \prime \prime}\right)=\frac{g^{\prime \prime \prime} 2}{8 \pi^{2}}\left(\begin{array}{ccccc}
-1 & 3 & 0 & 0 & 0 \\
8 / 3 & -2 / 3 & 2 / 9 & -1 / 9 & 1 / 3 \\
-11 / 3 & 11 / 3 & 22 / 9 & -2 / 9 & 2 / 3 \\
0 & 0 & 0 & 1 & -3 \\
-1 & 1 & 2 / 3 & -1 / 3 & -7
\end{array}\right)+0\left(g^{\prime \prime \prime} 4\right)
$$

The matrix $\mathrm{r}_{\mathrm{pr}}^{\prime \mathrm{IT}^{\mathrm{T}}}\left(\mathrm{g}^{\prime \prime \prime}\right)$ is dlagonaldzed by the transfarmation

$$
\sum_{p, r} x_{n p}^{-1} Y_{p r}^{\prime+1 r^{T}}\left(g^{\prime \prime \prime}\right) x_{r q}=\delta_{n q} Y_{q}^{\prime \prime \prime}\left(g^{\prime \prime \prime}\right),
$$

where

$$
x_{\mathrm{DP}}=\left(\begin{array}{ccccc}
.16866 & -.71636 & .052633 & .84853 & .69088 \\
-.16866 & .71436 & -.052633 & .56569 & -.69088 \\
-.050165 & -.030949 & -.16552 & -.28284 & -1.1681 \\
.028133 & .018728 & -1.0044 & 0 & .23229 \\
.73361 & .049722 & .35726 & 0 & -.17486
\end{array}\right), \text { (A.21) }
$$




$$
Y_{q}^{\prime \prime \prime \prime}\left(g^{\prime \prime \prime}\right)=\frac{g^{\prime \prime \prime 2}}{8 \pi^{2}}\left(\begin{array}{l}
-7.2221 \\
-3.7559 \\
1.0761 \\
2 \\
2.6797
\end{array}\right)+C\left(g^{\prime \prime^{4}}\right)
$$

Note that these eigenvaluas chack with those of Kef. B wherc the effective Hamiltonian for ocrangeness changing nonleptonic dccays was calculated in the four-quark model using a different nperator basis. The fourtn efgenvalun carregponds to the multiplicntively ronormalized su (3) 27 operator $3 Q_{1}+2 Q_{2}-Q_{3}$. This operator has both $I=1 / 2$ and $I=3 / 2$ pteces

$$
A_{q}^{\prime \prime \prime}=\left(\begin{array}{c}
-.80246 \\
-.41732 \\
.11957 \\
6 / 27 \\
.29774
\end{array}\right)
$$

The octet operators uoed in Ref, 8 wcre

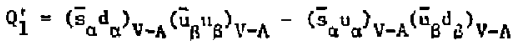

$$
\begin{aligned}
& Q_{2}^{\prime}=\left(\bar{s}_{\alpha} d_{\alpha}\right)_{V-A}\left(\bar{u}_{\beta} u_{\beta}\right)_{V-A}+\left(\bar{s}_{\alpha} u_{\alpha}\right)_{V-A}\left(\bar{u}_{B} d_{B}\right)_{v-A} \\
& +2\left(\bar{s}_{\alpha} d_{\alpha}\right) v_{-A}\left(\bar{d}_{\beta} d_{B}\right)_{v-A}+2\left(\bar{s}_{\alpha}{ }_{a}{ }^{a} v_{-A}\left(\bar{B}_{B}{ }^{B}{ }_{B}\right) v-A\right. \\
& Q_{5}^{\prime}=4\left(\bar{s}_{\alpha} T_{a B}^{a} d_{B}\right)_{v-A}\left[\left(\bar{u}_{a} I_{a B}^{a} u_{B}\right)_{v+A}\right. \\
& \left.+\left\{\bar{d}_{\alpha} T_{\alpha B}^{a} d_{B}\right\}_{V+A}+\left(\vec{s}_{\alpha} T_{a B}^{3} s_{B}\right)_{y+A}\right] \\
& Q_{b}^{f}=\left(\bar{s}_{a}^{d}\right)_{d} v_{-A}\left[\left(\bar{u}_{B} u_{B}\right)_{v+A}+\left(d_{B} d_{B}\right)_{v+A}+\left\langle\bar{s}_{B}^{A} B^{3} v_{V}\right]\right.
\end{aligned}
$$

where $T^{\text {a }}$ a $E(1, \ldots, 8\}$ are the $S U(J)$ color matrices normaltzed to $\operatorname{Tr}\left(T^{a} T^{b}\right)=\delta^{a b} / 2$. These operators can be written ln terms of the operators $Q_{1}, \ldots, Q_{6}$ of Chapter $I 1$ in the Eollowing manner

$$
\begin{aligned}
& Q_{1}^{\prime}=-Q_{2}+Q_{1} \\
& Q_{2}^{\prime}=-Q_{1}+Q_{2}+2 Q_{3} \\
& q_{5}^{\prime}=-\frac{2}{3} Q_{5}+2 Q_{6} \\
& q_{6}^{\prime}=Q_{5}
\end{aligned}
$$

(A. 25d)

Using these relations it can be show that the Anomalous dimension matrix in Eq. (A.19) agrees with that used In ReF, a. 


\section{APPEHDIX B}

In thlo appendix values are given for the various quantit 1 es which enter the computation of $\boldsymbol{x}_{3}$ fa portion of the effective Hamiltonian for

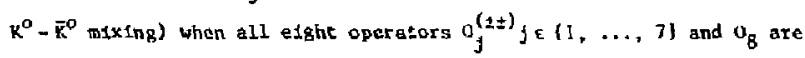
kept. These operators (def ined th Eqs. 18, 20 and 21 of chapter III) close under renormalization of the one loop level and thetr renormalization is characterized by the anonalous dimension matrices

$\left.Y_{1 j}^{\prime(+)}\left(g^{\prime}\right)=\frac{B^{2}}{B \pi^{2}}\left[\begin{array}{cccccccc}1 & 3 & 0 & 0 & 0 & 0 & 0 & 0 \\ 3 & 1 & -\frac{1}{9} & \frac{1}{3} & -\frac{1}{9} & \frac{1}{3} & 0 & 0 \\ 0 & 0 & \frac{7}{9} & \frac{11}{3} & -\frac{2}{9} & \frac{2}{3} & 0 & 32 \\ 0 & 0 & \frac{22}{9} & \frac{8}{3} & -\frac{5}{9} & \frac{5}{3} & 0 & 16 \\ 0 & 0 & 0 & 0 & 3 & -3 & 0 & -32 \\ 0 & 0 & -\frac{5}{9} & \frac{5}{3} & -\frac{5}{9} & -\frac{13}{3} & 0 & -16 \\ 0 & 0 & 0 & 0 & 0 & 0 & 4 & -24 \\ 0 & 0 & 0 & 0 & 0 & 0 & 0 & \frac{7}{3}\end{array}\right]+\cos ^{4}\right)$

(B.1)

$$
\left.r_{1 j}^{\prime(-)}\left(g^{\prime}\right)=\frac{8^{2}}{8 \pi^{2}}\left[\begin{array}{cccccccc}
-5 & 3 & 0 & 0 & 0 & 0 & 0 & 0 \\
3 & -5 & -\frac{1}{9} & \frac{1}{3} & -\frac{1}{9} & \frac{1}{3} & 0 & 0 \\
0 & 0 & -\frac{47}{9} & \frac{11}{3} & -\frac{2}{9} & \frac{2}{3} & 0 & -16 \\
0 & 0 & \frac{22}{9} & -\frac{10}{3} & -\frac{5}{9} & \frac{5}{3} & 0 & 0 \\
0 & 0 & 0 & 0 & -3 & -3 & 0 & 16 \\
0 & 0 & -\frac{5}{9} & \frac{5}{3} & -\frac{5}{9} & -\frac{31}{3} & 0 & 0 \\
0 & 0 & 0 & 0 & 0 & 0 & -8 & -8 \\
0 & 0 & 0 & 0 & 0 & 0 & 0 & \frac{7}{3}
\end{array}\right]+r^{\prime 4}\right)
$$

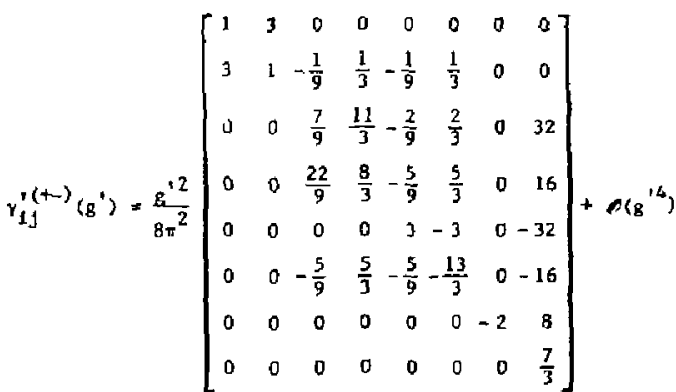

(B.3)

$$
r_{1 j}^{\prime(-)}\left(g^{\prime}\right)=\frac{g^{\prime 2}}{8 \pi^{2}}\left[\begin{array}{rrrrrrrr}
-5 & 3 & 0 & 0 & 0 & 0 & 0 & 0 \\
3 & -5 & -\frac{1}{9} & \frac{1}{3} & -\frac{1}{9} & \frac{1}{3} & 0 & 0 \\
0 & 0 & \frac{47}{9} & \frac{11}{3} & -\frac{2}{9} & \frac{2}{3} & 0 & -16 \\
0 & 0 & \frac{22}{9} & -\frac{10}{3} & -\frac{5}{9} & \frac{5}{3} & 0 & 0 \\
0 & 0 & 0 & 0 & -1 & -3 & 0 & 16 \\
0 & 0 & -\frac{5}{9} & \frac{5}{3} & -\frac{5}{9} & -\frac{31}{3} & 0 & 0 \\
0 & 0 & 0 & 0 & 0 & 0 & -2 & 0 \\
0 & 0 & 0 & 0 & 0 & 0 & 0 & \frac{7}{3}
\end{array}\right]+\rho\left(g^{\prime 4}\right)
$$

The tatrices $r_{1 j}^{\prime}( \pm t)^{T}\left(g^{\prime}\right)$ can be diagonalized by the transfomations

$$
\sum_{k, R} v_{i x}^{( \pm \pm)^{-1}} r_{2 k}^{( \pm \pm)^{T}}\left(g^{\prime}\right) v_{x\}}^{( \pm \pm)}=\delta_{t j} r_{j}^{( \pm \pm)}\left(g^{\prime}\right)
$$


where

$$
V_{k j}^{(++)}=\left[\begin{array}{cccccccc}
0 & .69589 & 0 & 0 & 0 & -.70658 & 0 & 0 \\
0 & -.69589 & 0 & 0 & 0 & -.70658 & 0 & 0 \\
-.20236 & -.23196 & .95985 & 0 & .17132 & .10094 & 0 & -.40226 \\
.28103 & .23196 & -.83058 & 0 & .083375 & .10094 & 0 & -.77672 \\
-.044316 & 0 & -.079869 & 0 & .96445 & 0 & 0 & .31309 \\
-.82989 & 0 & -.16334 & 0 & -.35439 & 0 & 0 & -.26431 \\
0 & 0 & 0 & 0 & 0 & 0 & 1 & 0 \\
-1.7593 & .85647 & -6.3181 & 1 & -23.46 & 2.9071 & -14.4 & -11.106
\end{array}\right]
$$

$V_{\mathrm{kg}}^{(--)}=\left[\begin{array}{cccccccc}0 & 0 & .69589 & 0 & 0 & -.70658 & 0 & 0 \\ 0 & 0 & -.69589 & 0 & 0 & -.70658 & 0 & 0 \\ -.20236 & 0 & -.23196 & .95985 & .17132 & .10094 & -.20226 & 0 \\ .28103 & 0 & .23196 & -.83058 & .083375 & .10094 & -.77672 & 0 \\ -.044316 & 0 & 0 & -.079869 & .96445 & 0 & .31309 & 0 \\ -.82989 & 0 & 0 & -.16334 & -.35439 & 0 & -.26431 & 0 \\ 0 & 1 & 0 & 0 & 0 & 0 & 0 & 0 \\ -.19115 & .77419 & -.35917 & 1.7372 & -2.4326 & .3727 & -3.5761 & 1\end{array}\right]$

$$
v_{k .}^{(+-)}\left[\begin{array}{cccccccc}
0 & 0 & .69589 & 0 & 0 & 0 & -.70658 & 0 \\
0 & 0 & -.69589 & 0 & 0 & 0 & -.70658 & 0 \\
-.201236 & 0 & -.23196 & .95985 & 0 & .17132 & .10094 & -.40226 \\
.28103 & 0 & .23196 & -.83058 & 0 & .083375 & .10094 & -.77672 \\
-.064316 & 0 & 0 & -.079869 & 0 & .96444 & 0 & .31309 \\
-.82989 & 0 & 0 & -.16314 & 0 & -.35439 & 0 & -.26431 \\
0 & 1 & 0 & 0 & 0 & 0 & 0 & 0 \\
-1.7949 & -1.0462 & .85646 & -6.3189 & 1 & -23.46 & 2.9071 & -11.106
\end{array}\right]
$$

(B.8)

$$
v_{k j}^{(-+)}=\left[\begin{array}{cccccccc}
0 & .69589 & 0 & 0 & 0 & -.70558 & 0 & 0 \\
0 & -.69589 & 0 & 0 & 0 & -.70538 & 0 & 0 \\
-.20236 & -.23196 & .95985 & .17132 & 0 & .10094 & -.40226 & 0 \\
.28103 & .23106 & -.83058 & .083375 & 0 & .10094 & -.77672 & 0 \\
-.044316 & 0 & -.079869 & .96665 & 0 & 0 & .31309 & 0 \\
-.82949 & 0 & -.16334 & -.05439 & 0 & 0 & -.26431 & 0 \\
0 & 0 & 0 & 0 & 1 & 0 & 0 & 0 \\
-.19119 & -.35917 & 1.3772 & -2.4326 & -1.8462 & .3727 & -3.5761 & 1
\end{array}\right]
$$


and

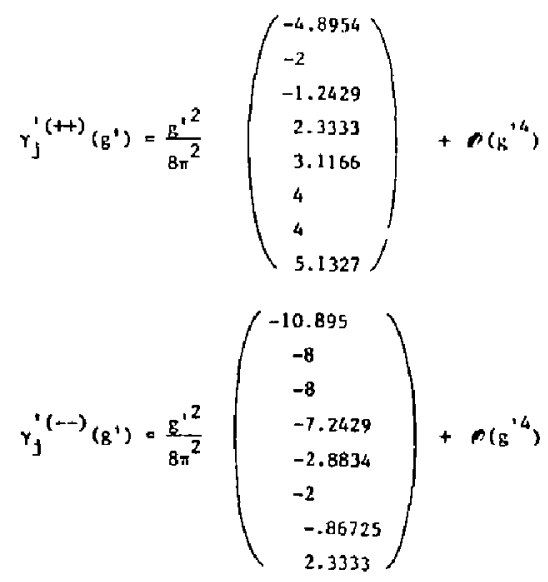

$Y_{f}^{\prime(+-)}\left(E^{\prime}\right)=\frac{g^{2}}{8 \pi^{2}}\left(\begin{array}{l}-4.8954 \\ -2 \\ -2 \\ -1.2429 \\ 2.3333 \\ 3.1266 \\ 4 \\ 5.1327\end{array}\right)+O\left(\mathrm{~s}^{14}\right)$

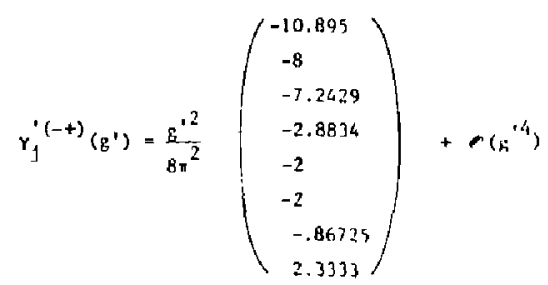

(B. 10)

(B.12)
At the stage of remoulfer the b-quark the same operators enter except that the factor $\mathrm{m}_{c}^{2} / \mathrm{g}^{2}$ in the deflndtion of $\mathrm{o}_{\mathrm{g}} 1$ s raplaced by $m_{c}^{\prime \prime 2} / \mathrm{g}^{\prime 2}$ and the b-quark field terms $1 \mathrm{~h} \mathrm{O}_{3}-\mathrm{U}_{6}$ are absent. Again these aperators close under strong interaction rennmalization. The calculacion of the anomalous dimension atrix is the rame as when the trquark was removed except it is calculated in an effuctive 4-quark thisory (instead of an effective 5-quark theory) of strong interactions with caupling g". The renuleing anomalous dimension matrices are:

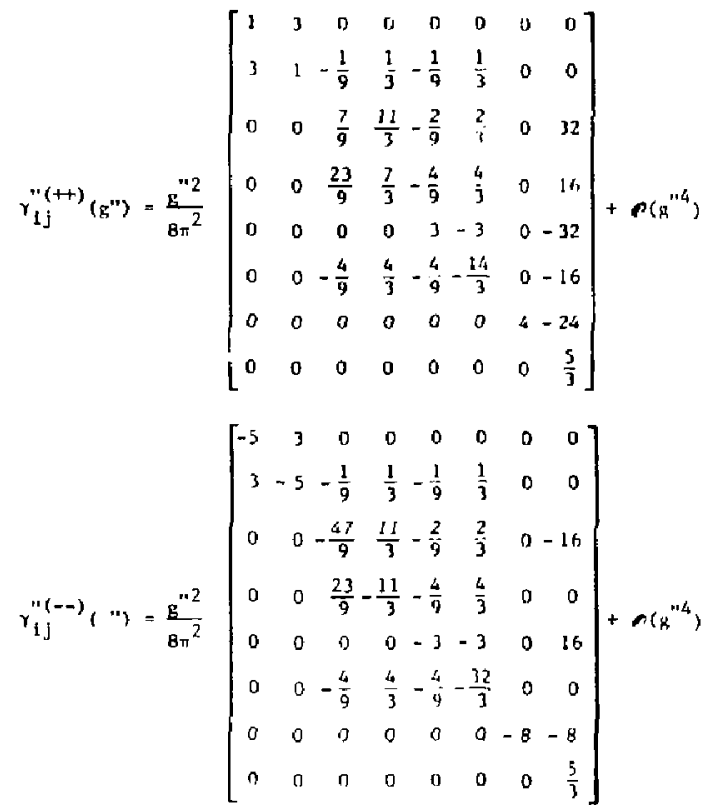




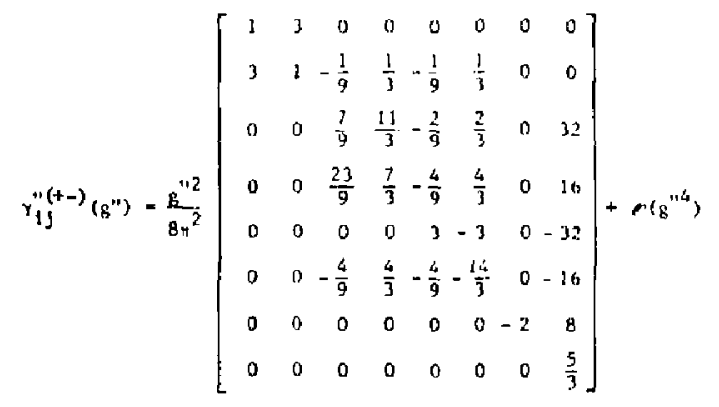

(B. 16)

$\left.x_{1 j}(-1)\left(g^{\prime \prime}\right)=\frac{8^{\prime \prime 2}}{\theta_{\pi}^{2}}\left[\begin{array}{cccccccc}-5 & 3 & 0 & 0 & 0 & 0 & 0 & 0 \\ 3 & -5 & -\frac{1}{9} & \frac{1}{3} & -\frac{1}{9} & \frac{1}{3} & 0 & 0 \\ 0 & 0 & -\frac{47}{9} & \frac{11}{3} & -\frac{2}{9} & \frac{2}{3} & 0 & -16 \\ 0 & 0 & \frac{23}{9} & -\frac{11}{3} & -\frac{4}{9} & \frac{4}{3} & 0 & 0 \\ 0 & 0 & 0 & 0 & -3 & -3 & 0 & 16 \\ 0 & 0 & -\frac{4}{9} & \frac{4}{3} & -\frac{4}{9} & -\frac{32}{3} & 0 & 0 \\ 0 & 0 & 0 & 0 & 0 & 0 & -2 & 8 \\ 0 & 0 & 0 & 0 & 0 & 0 & 0 & \frac{5}{3}\end{array}\right]+m^{(14}\right)$

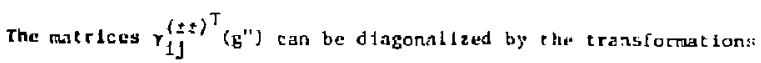

where

$W_{k J}^{(++)}=\left[\begin{array}{cccccccc}0 & .6578 & 0 & 0 & 0 & .70643 & 0 & 0 \\ 0 & -.6598 & 0 & 0 & 0 & .70643 & 0 & 0 \\ .14452 & -.3279 & -.74005 & 0 & .1414 & -.11774 & 0 & -.67961 \\ -.20298 & .3279 & .71236 & 0 & .067442 & -.11774 & 0 & -1.1468 \\ .032867 & 0 & .041364 & 0 & .96775 & 0 & 0 & .62931 \\ .72549 & 0 & .096564 & 0 & -.35021 & 0 & 0 & -.34187 \\ 0 & 0 & 0 & 0 & 0 & 0 & 1 & 0 \\ 1.6816 & 1.4308 & 5.1876 & 1 & -13.812 & -2.4221 & -10.286 & -14.961\end{array}\right]$

$G_{\mathrm{kJ}}^{(-)}=\left[\begin{array}{cccccccc}0 & 0 & .6558 & 0 & 0 & .70643 & 0 & 0 \\ 0 & 0 & -.6558 & 0 & 0 & .70643 & 0 & 0 \\ .14452 & 0 & -.3279 & -.78005 & .1414 & -.11774 & -.67561 & 0 \\ -.20298 & 0 & . .279 & .71236 & .067442 & -.11774 & -1.1468 & 0 \\ .032867 & 0 & 0 & .041364 & .96775 & 0 & .42931 & 0 \\ .72549 & 0 & 0 & .096564 & -.35021 & 0 & -.34187 & 0 \\ 0 & 1 & 0 & 0 & 0 & 0 & 0 & 0 \\ .14055 & .82759 & -.54273 & -1.4336 & -2.8936 & -.51377 & -6.3689 & 1\end{array}\right]$

(B. 20)

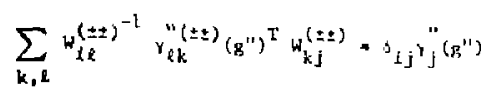

(B.18) 
$H_{\mathrm{kJ}}^{(+-)}=\left[\begin{array}{cccccccc}0 & 0 & .6558 & . & 0 & 0 & .70643 & 0 \\ 0 & 0 & -.6558 & 0 & 0 & 0 & .70643 & 0 \\ .14452 & 0 & -.3279 & -.78005 & 0 & .1414 & -.11774 & -.67561 \\ -.20298 & 0 & .3279 & .71236 & 0 & .067442 & -.13774 & -1,1468 \\ .032867 & 0 & 0 & .041364 & 0 & .96775 & 0 & .62931 \\ .72549 & 0 & 0 & .096564 & 0 & -.35021 & 0 & -.34187 \\ 0 & 1 & 0 & 0 & 0 & 0 & 0 & 0 \\ 1.6816 & -2.1818 & 1.4308 & 5.1876 & 1 & -13.912 & -2.4221 & -16.961\end{array}\right]$

(0.2)

$w_{k j}^{(-+)}=\left[\begin{array}{cccccccc}0 & .6558 & 0 & 0 & 0 & .70643 & 0 & 0 \\ 0 & -.6558 & 0 & 0 & 0 & .70643 & 0 & 0 \\ .14452 & -.3279 & -.78005 & .1618 & 0 & -.11774 & -.67561 & 0 \\ -.20298 & .3279 & .71236 & .067442 & 0 & -.11774 & -1.1668 & 0 \\ .032867 & 0 & .041364 & .96775 & 0 & 0 & .42931 & 0 \\ .72549 & 0 & .096564 & -.35021 & 0 & 0 & -.34187 & 0 \\ 0 & 0 & 0 & 0 & 1 & 0 & 0 & 0 \\ .14055 & -.54273 & -1.4336 & -2.8936 & -2.1818 & -.51377 & -6.3689 & 1\end{array}\right]$

(B. 22) and
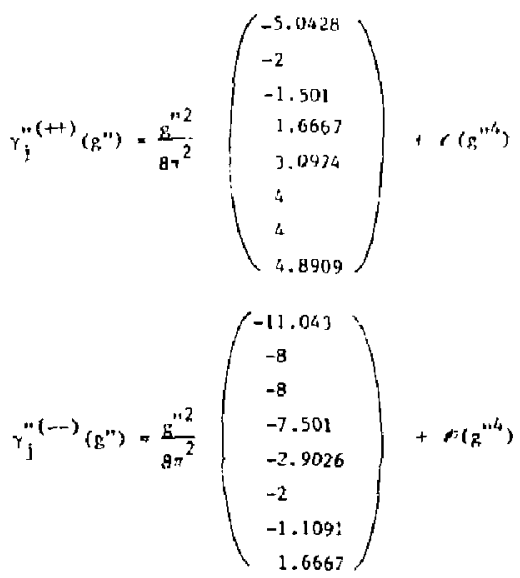

(B. 24)

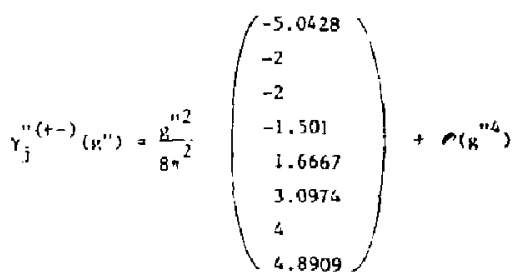

(3.25)

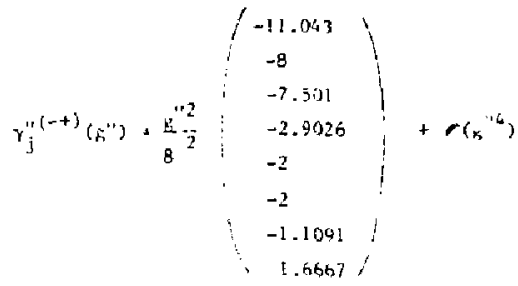


At the stage of removing the c-quark only the operator $m_{c} 2(\bar{s} d) v-A$ (Ğd) $V-A$ appears. It is mulfiplicat tvely renormalized (1.e., does not aix with other operators) and has the anomalous dimenston $\mathrm{B}^{\prime 2} / \mathrm{s}^{2}+$ $\mathrm{g}^{112 / 4 \pi^{2}}$ to leading order in the strong coupling.
APPEALIX $\mathrm{C}$

Here some useful $5 \mathrm{~b}(3)$ relations are given. The Gell Mann matri$\operatorname{ces} \lambda_{1}, \operatorname{le}\{1, \ldots 8\}$ are

$x_{1}=\left(\begin{array}{lll}0 & 1 & 0 \\ 1 & 0 & 0 \\ 0 & 0 & 0\end{array}\right), x_{2}=\left(\begin{array}{ccc}0 & -1 & 0 \\ 1 & 0 & 0 \\ 0 & 0 & 0\end{array}\right), x_{3}=\left(\begin{array}{ccc}1 & 0 & 0 \\ 0 & -1 & 0 \\ 0 & 0 & 0\end{array}\right), x_{4}=\left(\begin{array}{lll}0 & 0 & 1 \\ 0 & 0 & 0 \\ 1 & 0 & 0\end{array}\right)$

$x_{5}=\left(\begin{array}{ccc}0 & 0 & -1 \\ 0 & 0 & 0 \\ 1 & 0 & 0\end{array}\right), x_{6}=\left(\begin{array}{lll}0 & 0 & 0 \\ 0 & 0 & 1 \\ 0 & 1 & 0\end{array}\right), x_{7}=\left(\begin{array}{ccc}0 & 0 & 0 \\ 0 & 0 & -1 \\ 0 & 1 & 0\end{array}\right), x_{8}=\frac{1}{\sqrt{3}}\left(\begin{array}{ccc}1 & 0 & 0 \\ 0 & 1 & 0 \\ 0 & 0 & -2\end{array}\right)$

These matrices form the generators of SUi(3). In addition they transform as a basls for the adjoint octet representation. The spin $1 / 2$ ground state posicive parity baryons also transform as a basis for the

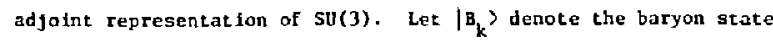
with the same SU(3) quantum numbers as $k_{k}$ - Then

$$
\begin{aligned}
& \left|E^{+}\right\rangle=\frac{1}{\sqrt{2}}\left|B_{1}+\mathrm{IB}_{2}\right\rangle \\
& \left|\Sigma^{-}\right\rangle=\frac{1}{\sqrt{2}}\left|\mathrm{~B}_{1}-\mathrm{IB}_{2}\right\rangle \\
& \left|\varepsilon^{\circ}\right\rangle=\left|\mathrm{B}_{3}\right\rangle \\
& \left|p^{\prime}\right\rangle=\frac{1}{\sqrt{2}}\left|\mathrm{~B}_{4}+\mathrm{IB}_{5}\right\rangle
\end{aligned}
$$




$$
\begin{aligned}
& |\mathrm{n}\rangle=\frac{1}{\sqrt{2}}\left|\mathrm{~B}_{6}+\mathrm{iB}_{7}\right\rangle \\
& \left|\mathrm{Z}^{\mathrm{O}}\right\rangle=\frac{1}{\sqrt{2}}\left|\mathrm{~B}_{6}-1 \mathrm{~B}_{7}\right\rangle \\
& \left|\Xi^{-}\right\rangle=\frac{1}{\sqrt{2}}\left|\mathrm{~B}_{4}=\mathrm{iB}_{5}\right\rangle \\
& \left|\Lambda^{0}\right\rangle=\left|\mathrm{B}_{8}\right\rangle
\end{aligned}
$$

The matrix element for an operator $o_{k}$ which transfoms like the $k$ 'th component of an octet $\left(i+e .\right.$, like $\left.\lambda_{k}\right)$ under su(3) is given by

$$
\left\langle B_{i}\right| a_{k}\left[B_{j}\right\rangle=d_{k i j} d-i f_{k 1 j} F
$$

The non-zero $d_{i j k}$ and $f_{i j k}$ are listed in Table VII.
REFERENCES

1. Particle Daca Group. Phys. Lete. 758, 1, (1978)

2. S. Welnberg, Phys. Rev. Lett, 19, 1264 (1967): A. Salam, in: Elementary Particle Thearg: Relativistic Groups and Analyticity (Nobnal Sympostum No. 8), editer by N. Svartholm (Almquist and Wikse11, Stockholm, 2968) p. 367.

3. K. G. Wilsen, Phys. Kev. 179, 1699 (1969).

4. For a review see W. Marctano and H. Pagcls. Phys. Reports 36, 137 (1978).

5. M. K. Galllard and B. W. L.ee, Pliyg. Rev. Lect. 33, 108 (1974).

6. G. Nltarelli and L. MalanL, Phys. Letc. 52B, 351 (1974).

7. M. B. Wise and E. Witten, Phys. Rev. $\underline{220}, 1216$ (1979),

8. A. I. Vainshte1n, V. T. Zakharov, and M. A. Shifman, JETP Lett. 22, 55 (1975); M. A. Shifman, A. I. Valnshcein, and V. I. Zakharov. Nucl. Phys. B120, 316 (1977); and [TEP-63, 64 (1976) unpub1lshed.

9. Ie 15 possible to add extra Hitge so thac CP violation occurs in the 4-quark model. See T. D. Let, Phys, Rev. D8, 1226 (1973); Phys. Rep. 9C, 143 (1976); S. Welnberg, Phys. Rev. Lett. 37, 657 (1976); P. Sikivie, Phys. Lete, 65B, 141 (1976).

10. M. koboyoshit and I. Maskawa, Pros. Thear. Phys. 49. 652 (1973).

11. For a revtew seg M. Per1. Slac-puB-2446 (1980) unpublished.

12. The phenomenology of $b$ and $t$ quarks is Jisiussed in J. Ellis, M. K. Gatllard, D. V. Manopoulus and $S$, Rudaz, Nucl. Physs. B131, 285. (1977). 
13. It is not however necessary. For bxamples of models without $]$ t-quark see H. Georgl and S. L. Glashiow, Harvard Universicy proprinc 9/A073 (1979) umputished.

14. S. L. Glashow, I. Iltopoulos, and l. Matani, Phys. Rev, D? (1970 1285 .

15. C. Bouchiat et al.. Phys, Letc, 38B, 519 (1972) and D. J. Gross and R. Jackiw, Phys. Rev. D6, 477 (1972).

16. F. J. Gilman and M. B. Wise, Phys, teote. 8.3., 83 (1979),

17. J. Elits, M. K. Gaillard, and D. V. Nanopoulos, Nucl. Phys tyl09, $213(1976)$

18. The results of this chapter appear in: F. J. Gilman and M. B. Hise, Phys, Rev, D20, 2392 (1979).

19. E. Witten, Nucl. Phys. B104, $445(1976)$.

20. E. Witren, Nucl. Phys. B122, 109 (1977).

21. S. Weinberg, Phys. Rev. De, 3497 (19/3); G. 'c Hoof t, Nucl. Phys. B6.]. 445 (1973).

22. M. Gell-Mann and F. Low, Phys. Rev. 95, 1300 (1954); E. C. G. Stueckelberg and E. PLeerman, helv. Phys. Acta 26,499 (1953): C.G.Callan, Phys. Rev. 2ㅡ, $1 \$ 41$ (1970); K. Symanzik, Comrusu. Math. Plyys. 18, 227 (1970).

23. D. J. Gross and F. W1lczek, Phys. Rev, Lett. 30, 1343 (1973); H. D. Politzer, 占bdd. 30, 1316 (1973).

24. The running fine-structure constants are defined by $a\left(\mathrm{M}^{2}\right)$ $=8(M / \mu, g)^{2} / 4 \pi$ so that $a\left(\mu^{2}\right)=g^{2} / 4 \pi$. Stmilarly $a^{\prime}=-\bar{g}, 2 / 4 \pi$, $a^{\prime \prime}=\bar{g}^{\prime} \cdot 2 / 4 \pi$, and $a^{\prime \prime}=\bar{g}+1{ }^{2} / 4 \pi$.
25. T. Arrelquise and I. Carrazone, Phys. Kev. D4, 2856 (1975).

26. A detailed discussion of what is meant by an effective theory of strong interictions can be found in Secs. 2 and 3 of Ref. 19.

27. J. G. H. DeGroot et a1., Phys, Letc. 82B, 292 (1979); 82B, 456 (1979): 2. Phys. Cl, 142 (1979).

28. For a ruview sce: J. D. Jackson, C. Diulgg, and J, L. Rosner in Proceed ings of chw 19th Internarional Conference on High Energy Physics, Tokyo, 1978, adited by S. Homna, M. Kauagucht, and H. M1yazark (Phys. Soc. Japan, Tokyo, 1979) 391.

29. Thls paine has been emphaslaed in Ref. $S$ and in $J$. EIl1s, M. $K$. Gaillard and D. Y. Nanopoulas, Nucl. Phys. BlOG, 313 (1975).

30. The matrix elements of $Q_{5}$ also have the chiral structure $(V-A) \odot(V+A)$ whtch leads to enhanced matrix elements. HoLever, the Wilson coeffictent $c_{5}$ is much smaller than $c_{6}$ and in the vacuum insertion approximation the matrix elements of $Q_{5}$ are suppressed by a faccor of $1 / 3$ compared to those of $Q_{6}$.

31. This chapter is an expanded version of: F. I. Gilman and M. B. Wise, SLAC-FUB-2473 (1980) (co appear in Phys. Lett.)

32. M. K. Galllard and B. W. Lee, Phys. Rev, D16, 897 (1974)

33. A. I, vainshte1n, V. I. Zakharov, V. A. Novikov, and M. A.Shtfman, Sov. J. Nucl. Phys. 23, 540 (1976); E. Witten, Kuc1. Phys, B122. 109 (1977); V. A, Novfikov, M. A. Shifman, A. I. Vainshcein, and V. 1. Zakharov, Phys, Rev, D16, 223 (1977)

34. D. v. Nanopoulos and C. G. Ross, Phys. Let. $\underline{56}, 1219$ (1975); A. I. Vajnshteln, V. C. Zakharov, V. A. Novitov, and M. A. Shifman, Phys. Lett. 60B, 71 (1975) 
35. The advantages of using the defintition were discussed in detali by F. J. G11min and M. B. Wise, SLAC-PUB-2439 (1979) to appear In Phys. Rev. D.

36. R. F. Streater and A. S. Wightman, PCT Spin Statisties and ALl That (Benjamin/Cumming, Publishing Co., sew York, 1964).

37. K. Kleinknecht, In Proceedings of the XVII Internaclonal conference on High Energy Physics, London, 1974, edited by J. R. Salth (Rutherford Laberatory, Chllton. Didcot, Berkshire. England, 1974) P. [II-23.

38. F. J. Cllowan and H. B. Wise, Phys, Rev D20, 2392 (1979); B. D. Ga1ser, T. Tsao and M. B. Hise, SLAC-PUB-2523 (1980) unpublished,

39. J. S. Hagelin, Harvard Universicy Preprint 80/A018 (1980) unpublished.

40. V. V. Prokhorov, Yidd. Fiz. 吕, 1111 (1979); B. Cuberina and R. D. Pecce1, Nucl. Phys, B163, 289 (1980); f. S. Hageltin, Harvard liniversily Preprint 79/A081 (1979) unpublislied.

41. R. Bernstein, I. H. Cronin, B. Winstein, B. Cousins, J. Greenhalgh, M. Schuart2, D. Hedin, and G. Thomson, Fermilat experiment E-6:7.

42. L. Walfenstein, Phys. Rev. Lett. 13, 562 (1964).

43. Some of the work presented in this chaptar was published in F. J. G1lman and M. B. WIse, Phys. Rev. D19, 976 (1979).

44. M. K. Galllurd, Il Nuovo Clmento 6A, 559 (1971); G. R. Farrar, Phys. Rev. D4, 212 (1971); R. Gavroglu and H. P. W. Gottle1b, Nuc1. Phys. B79, 168 (1974); J. O. Eegr, University of 0slo,
Insticure of Physics Report 79-09 (1979); M. A. Shifman, A. I. Vatnshtein, and V. 1. Zakarov, Phys. Rev. D18, 2583 (1978).

45. N. Vas int i, Phys, Rev, D13, 1889 (1976); M. A. Ahmed and G. G. Rass, Phys. Lett. 59트 (1975).

46. For a revicw see J. J. S. Kokkedee, The Quark Model (w. A. Benjamin Inc., New York. 1969).

47. H. Jacob and C. C. Hich, Ann. Phys. $\underline{7}, 404$ (1959),

48. W. Yeh et al.. Plys, Rey. 이, 1545 (1974); and $M$. Bourquin ec al., Phys. Lete. 88B, 192 (1979)

49. M. A. Shifman, A. I. Vainshtein and V. T. Zakharov, Phys. Rev. D18, 2583 (1978) and 1TEP preprine ITEP-113 (1976) unpublished.

50. For a revieu see $\mathrm{s}$. B. Trelman in: Lectures on Curregk Algebra and Its Appltattons (Princeton University Press, Princeton, 1970).

51. This follows from the Eact that the oucer praduce of two octets contains the octet representation twice, $1 . t .888=$ $1 \oplus 8 \oplus 8 \oplus 10 \oplus \overrightarrow{10} \oplus 27$.

52. A contribution from the 27 part of the uffective Hamlltonian for is = 1 weak nopleptonic decays implites a vlolation of the LeeSugayara relation. See: R. W. Lee, Phys. Rev. Lett. 12, 83 (1964); H. Sugauara, Prog. Theur. Phys. 31, 213 (1964).

53. L. F, Abbott and M. B. Wise, S.AC-PLB-2482 (1980) unpublislued.

54. For some recent work seE S. Weinberg. Harvard University preprint 80/A001 (19B0) unpublished; B. Ovrut and $H$. Schftzer, Brandels Undversity preprint (1980) unpublished; L. Hall, Harvard Lniversicy preprint (In prenaration); L. F, dbbott and $Y .8$. Hise. SLAC-PUB-2487 (1980) unpublished. 
55. In princtiple the accuracy of our treatment can be improved by golng beyond the leading logartthme approximation, calculating the two loop atamalous demenstorts and one loop fnithal value for the Wilson cocffictents.

56. J. F. Danoghue, E. Golowich, W. A. Ponce, and B. K. Holgtein, Phys. Rev. D21, 186 (1980). 